\title{
Chimarra of Sabah and Sarawak, northern Borneo (Trichoptera: Philopotamidae)
}

\author{
Roger J. Blahnik, Ralph W. Holzenthal \& Jolanda Huisman
}

\begin{abstract}
Thirty new species of Chimarra Stephens (Trichoptera: Philopotamidae) from the Malaysian part of Borneo (Sabah and Sarawak) are described, and eight previously described species are redescribed (Chimarra dulitensis Kimmins, 1955 and C. demeter Malicky, 2000, originally described from the Malaysian part of Borneo; and also C. phlegyas Malicky, 2008; C. polyneikes Malicky, 2008; C. prokrustes Malicky, 2008; C. terramater Malicky, 2008; C. thaumas Malicky, 2008; and C. tityos Malicky, 2008, originally described from the Indonesian part of Borneo, but now also recorded from the Malaysian part of Borneo). New species described include: Chimarra antheae, C. caduca, C. chanchuluni, C. cuspidata, C. cygnus, C. danumensis, C. dejongi, C. denticula, C. devogeli, C. drepane, C. fuilianae, C. gyrospina, C. jannekae, C. karlijnae, C. kinabaluensis, C. lambi, C. liwaguensis, C. noloyan, C. noohi, C. phillipsae, C. physanoton, C. preapicalis, C. scolops, C. silausilau, C. sinitorum, C. stenodactylus, C. vantoli, C. vanwelzeni, C. ventritropis, and C. xiphosella. Two major groups, the Chimarra tsudai group and the Chimarra digitata group are recognized, based on characters of the male genitalia and wing venation, to include most of these species.

Roger J. Blahnik, Department of Entomology, 1980 Folwell Ave., 219 Hodson Hall, University of Minnesota, St. Paul, Minnesota, 55108, U.S.A. blahn003@umn.edu Ralph W. Holzenthal*, same address, holze001@umn.edu Jolanda Huisman, same address, jolandahuisman01@gmail.com
\end{abstract}

\section{Introduction}

Currently 229 species of Chimarra are known from the Oriental region, of the approximately 630 species known for the world. The vast majority of these have been described in just the last 20 years. Until the recent paper by Malicky (2008), only three species were previously described from Borneo: Chimarra atripennis Banks, 1931, C. dulitensis Kimmins, 1955 and C. demeter Malicky, 2000. The seven additional species recently described by Malicky from the Indonesian part of Borneo (Eastern Kalimantan) include C. phlegyas, C. polyneikes, C. prokrustes, C. terramater, C. thaumas, C. thyestes, and C. tityos. Chimarra tawitawi Malicky, originally described from the Philippines (Malicky 1994), was also reported from Kalimantan by Malicky (2008). All of the described species, except $C$. thyestes and
C. tawitawi also occur in the northern, Malaysian part of Borneo. This paper thus redescribes and reillustrates all of the species currently known from Borneo, excluding only $C$. atripennis, which is based on an unassociated female holotype, and $C$. thyestes and $C$. tawitawi which have not yet been collected from the Malaysian part of Borneo. It includes an additional 30 new species from the Malaysian states of Sabah and Sarawak, bringing to 40 the number of species now recorded from Borneo, all of them endemic, except $C$. dulitensis, which has also been reported from Sumatra by Malicky (1989), and C. tawitawi, otherwise only known from the Philippines. It should be noted, however, that one of the new species described here ( $C$. devogeli, sp. n.) is very similar to $C$. tawitawi, and it is possible that the record of $C$. tawitawi from Kalimantan may 
actually be $C$. devogeli. Despite the known diversity of Chimarra in the Oriental region, the overall number of species will undoubtedly expand considerably in the future, particularly with the description of the fauna from India and other under-collected regions, including the Indonesian part of Borneo. The species from northern Borneo are, in general, most similar and undoubtedly most closely related to species from Southeast Asia, particularly those recently described from Thailand and Vietnam (Chantaramongkol \& Malicky 1989; Malicky \& Chantaramongkol 1993a, 1993b, 1997, 2003; Malicky 1994, 1995; Malicky \& Prommi 2006; Malicky et al. 2000, 2005, 2004, 2006; Mey 1998a, b). As discussed below, most of these fall into two distinctive species groups, the Chimarra tsudai group and the Chimarra digitata group, based on characters of the male genitalia and wing venation.

Most of the material studied for this research was collected by Jolanda Huisman between June, 1986 and May, 1987 in Sabah, Sarawak, and Brunei, during which time some 200 collection sites were visited. Habitats from which these collections were made ranged from lowland to montane $(50-3300 \mathrm{~m})$ primary forest; Chimarra species occurred between 50-1900 m.

\section{Materials and methods}

Most specimens examined were cleared in $10-12 \%$ $\mathrm{KOH}$, overnight at room temperature; additional specimens were cleared in lactic acid according to the method described by Blahnik \& Holzenthal (2004) and Blahnik et al. (2007). Specimens were examined and illustrated using an Olympus SZX12 stereomicroscope at magnifications up to $200 \times$. Drawings were made by use of a $10 \times 10 \mathrm{~mm}$ ocular grid and inked on a light table. Terminology for genitalia and wings follows that used by Blahnik (1998) for Chimarra. Female identifications are based on their co-occurrence with males and similarity in size or coloration; they are included in the type series because of the value of having presumptively associated material for future studies. The word Sungai (abbreviated Sg.) used in the locality data is the Malay word for a river or stream. Holotypes are deposited in the Nationaal Natuurhistorisch Museum Naturalis (formerly Rijksmuseum van Natuurlijke Historie), Leiden, The Netherlands (RMNH). Paratypes are deposited in the same institution and also in the collections of the University of Minnesota, St. Paul, Minnesota, USA (UMSP), the United States National Museum of Natural History, Washington, DC, USA (NMNH), and the Museum für Naturkunde, Humbolt-Universität, Berlin, Germany (MNHU).
The material was predominantly collected with light traps and preserved in 70\% ethyl alcohol, transferred to $80 \%$ ethyl alcohol on final curation. Some material from the collections of the NMNH and MNHU is preserved on pins. Material collected by Wolfram Mey and deposited at the MNHU was identified by him based on illustrations provided by the authors, but was not personally examined by the authors. It was added to the type material to extend the distributional records for the species and to make known the availability of this material for subsequent associative and taxonomic work.

\section{Systematics}

Most of the species of Chimarra from Sabah can be divided into two distinctive groups. These correspond to "lineages" discussed by Ross (1956), and characterized by the species $C$. digitata Martynov and C. tsudai Ross. Both groups, as is typical of the species currently placed in the subgenus Chimarra, are characterized by male genitalia with tergum $\mathrm{X}$ divided and widely separated mesally into paired, sclerotized lateral lobes (Blahnik 1998, fig. 9A-B). Both groups are widespread and species diverse in Asia, with members of the digitata group also occurring in the New World, Africa, and extending into some Pacific islands and eastern Australia. Additional "lineages" were also recognized by Ross (1956), including his Vigarrha and Chimarrhafra lineages from the Philippines and Africa, respectively, based on genera that Ross synonymized under Chimarra. Blahnik (1998) provided evidence that all of these Old World species groups belong in the subgenus Chimarra. However, some species in the subgenus Chimarra, including three included in this paper, are not readily placed in any of these groups. These may either be primitive or aberrant species within the major groups discussed here, or representatives of additional species groups. Two of these species, C. polyneikes Malicky and C. noohi, sp. n., lack a curved and inflected stem of the Rs vein of the forewing and also have the $s, r-m$, and $m$ crossveins of the forewing linearly aligned and hyaline (Figs 44A, 45A). Based on this set of wing venational characters, these two species are similar to species in Ross's Chimarrhafra group of the subgenus Chimarra, and also to species in the genus Chimarrhodella Lestage and the New World endemic Chimarra subgenera, Curgia Walker, Otarrha Blahnik, and Chimarrita Blahnik. However, like other species in the subgenus Chimarra, the two new species from Borneo have tergum $\mathrm{X}$ divided into widely separated lateral lobes. This character evidence suggests that these species may be basal or primitive species within the subgenus Chimarra, and hence 
of special interest because of their value for inferring phylogenetic relationships within the subgenus. Various species from other geographical regions undoubtedly also have primitive venational characteristics, but this information is not always included in species descriptions.

The tsudai group, as recognized here, is well-defined and the species belonging to it are generally easy to discern from literature descriptions. It is given formal recognition here as the Chimarra tsudai group. Species belonging to this group are listed in Table 1 and the characters defining it are discussed below. The membership of the digitata group is more difficult to define, in part because the characters defining it are not always easy to assess from species descriptions. Thus, although we discuss the general characters defining the group below, we have not attempted to provide a list of the included taxa. In a larger sense, the digitata group undoubtedly falls within a more inclusive group of species within which the characters defining the digitata group emerged. This larger group includes the type species for the genus, Chimarra marginata (Linnaeus, 1767). This is evidenced by the similar venational characters $C$. marginata shares with the digitata group. However, among other characters, C. marginata lacks the pair of elongate endothecal spines that generally characterizes the digitata group. This more comprehensive group is referred to here as the marginata group. Its recognition is useful in that it clearly defines the subgenus Chimarra, in the event that additional subgenera are eventually recognized from within the subgenus as currently defined. We have restricted the definition of the digitata group to species with the set of apomorphies discussed by Ross (1956) for Chimarra digitata and by Blahnik (1998) for New World representatives of the subgenus Chimarra. Other characters are discussed below. Some of the other Asian species discussed by Ross, such as C. sepulchralis Hagen and C. circularis Hagen, share this same basic set of characters and could be considered members of this group. We have retained $C$. digitata as a name to refer to this group because it lacks distinctive secondary apomorphies and is more representative of the morphology of the group as a whole. New World members of the Chimarra digitata group were placed into a number of small, well-defined species groups (Blahnik 1998). These can be considered subgroups of the digitata group, as defined here. Australian species of Chimarra, some of which can be placed in the Chimarra digitata group, were also organized into informal species groups by Cartwright (2002). As discussed below, a number of species from Borneo belong to the digitata group. Ultimately, these will probably also be organized into a number of subgroups, but this will undoubtedly require a comparative study of all of the Asian species as a preliminary step.

\section{Chimarra digitata group}

Ross (1956) used C. digitata to characterize a lineage of Chimarra with a character set that included, in the male genitalia, exactly two sensilla on the lateral lobes of tergum $\mathrm{X}$ and an apically divided membranous mesal lobe. He proposed that a member of this lineage gave rise to the Chimarra aterrima lineage (New World members of the subgenus Chimarra). Blahnik (1998) discussed additional characters applicable to New World species, some of which also apply to Old World species, including $C$. digitata. These include well-developed anterodorsal apodemes on segment IX and the presence of two elongate, basal, and usually asymmetrically arranged endothecal spines (varying from 1-3, rarely absent). Since there is no evidence that New World species in the subgenus Chimarra constitute a monophyletic group, the Chimarra aterrima group is here subsumed within the Chimarra digitata group. Venational characters typifying this group, and also applicable to C. marginata, include a sinuously inflected forewing Rs vein, such that the basal fork of the discal cell is distinctly asymmetrical, a short discal cell (length usually about 2 times width), and forewing $2 \mathrm{~A}$ vein intersecting $3 \mathrm{~A}$, and with a crossvein to $1 \mathrm{~A}$ vein proximal to the intersection (making the apex of $2 \mathrm{~A}$ appear forked or Y-shaped) (Fig. 40A). The description below applies to most of species of the Chimarra digitata group; individual species may vary in one or more characters.

Adult. Forewing with pronounced, sinuous inflection of Rs, sometimes with enlarged node at point of inflection, extending into cell below; base of discal cell forked asymmetrically, usually distinctly thickened and enlarged; discal cell short (length usually $2-2.5$ times width); $m$ crossvein proximal to $s$ and $r-m$ crossveins, $s$ not hyaline; hind wing 2 A complete, crossvein to 1A present (apparently forked apically). Male foretarsi and claws enlarged or not.

Male genitalia (Figs 2, 7). Segment VIII not highly modified, tergum often slightly longer than sternum, sternum sometimes with short ventral process. Segment IX with anteroventral margin at least somewhat expanded, sometimes distinctly so, mesal margin sinuously invaginated or broadly rounded; anterodorsally, with pair of distinctly projecting apodemes; dorsal margin short, but present, continuous posteriorly with projecting membranous mesal lobe of tergum X; ventral process posteriorly 
a

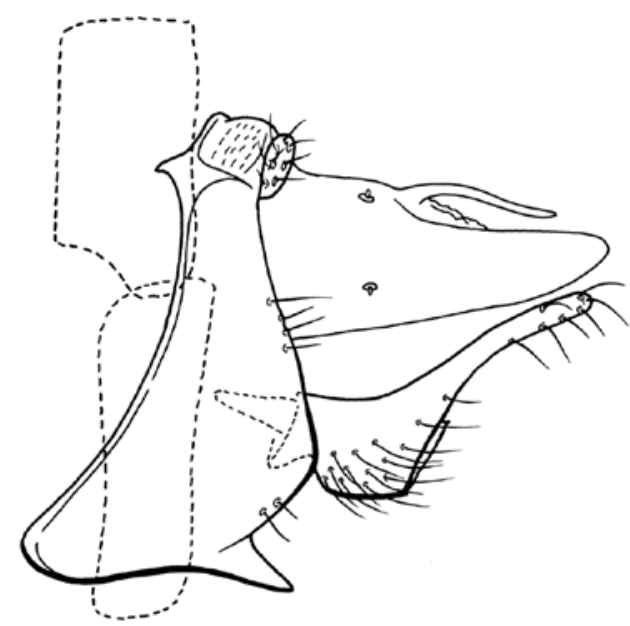

b

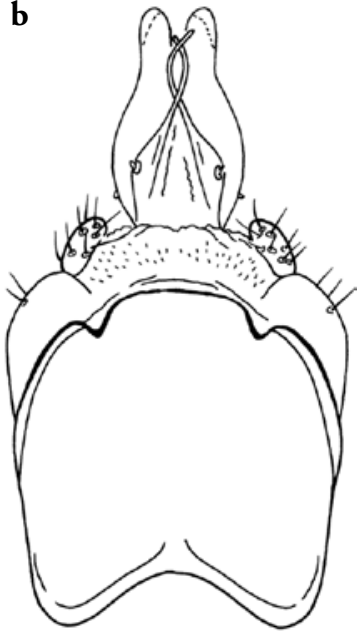

c

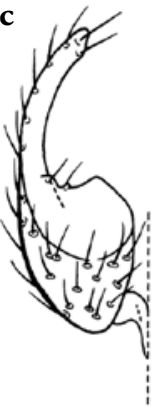

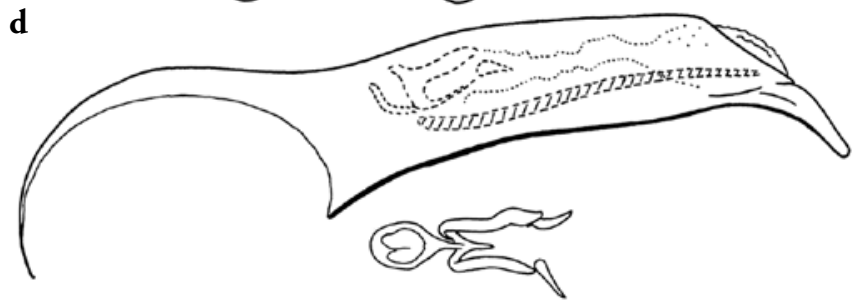

Fig. 1. Chimarra antheae, male genitalia. - a, lateral; b, segment IX and tergum X, dorsal; c, inferior appendage, ventral; $\mathrm{d}$, phallic apparatus, lateral; inset, dorsal of phallotremal sclerite complex.

projecting, varying from short, triangular to long, acute. Membranous mesal lobe of segment X usually divided apically, secondarily sclerotized in a few species, never with sensilla. Lateral lobes of tergum $\mathrm{X}$ sclerotized, widely separated, usually more or less vertical in orientation; sensilla reduced to 2 (nearly consistently), often on projecting process. Preanal appendages short, setose, often somewhat flattened. Inferior appendages extremely variable in shape in different species, usually with strong basal inflection, as viewed laterally, and long (or short) dorsal projection, dorsal projection usually distinctly narrower than basal part of appendage; in ventral view, with distinct mesal curvature, often with mesal ridge, tooth, tubercle, or spine-like projection from mesal surface. Phallobase typically with slight constriction near base, forming slight bulge or "belly"; ventral apex usually projecting (at least slightly). Endotheca with 2 short to elongate endothecal spines, asymmetrically arranged near base of structure (nearly symmetrically arranged in a few species, reduced to one in others, or with a $3 \mathrm{rd}$, much reduced and more apical spine in a few species); endotheca often elongate and much retracted into phallobase when not inflated; apex textured, or with small spines or papillate projections in most species. Phallotremal sclerite complex typically composed of simple rod and ring structure, usually much shorter than either phallotheca or endotheca, sometimes with accompanying dorsolateral or apicolateral sclerites.

\section{Chimarra antheae sp. $\mathrm{n}$.}

Fig. 1

Type material. Holotype: $\widehat{\delta}$, Malaysia, Sabah, Kinabalu National Park, Headquarters, roadside, $06^{\circ} 00^{\prime} \mathrm{N}, 116^{\circ} 32^{\prime} \mathrm{E}, 1500 \mathrm{~m}, 12 . v .1987$, J. Huisman, (UMSP000208644) (RMNH).

Paratypes. Malaysia, Sabah, $3.5 \mathrm{~km} \mathrm{SW} \mathrm{Kg.} \mathrm{Long}$ Pa Sia, Sg. Ritan, $04^{\circ} 24^{\prime} \mathrm{N}, 115^{\circ} 42^{\prime} \mathrm{E}, 1160 \mathrm{~m}, 8-$ 9.iv.1987, J. Huisman, 10 , 19 (UMSP); Crocker Range, $5 \mathrm{~km} \mathrm{~N}$ Tenom, base of $\mathrm{Sg}$. Ulu Noloyan, $05^{\circ} 10^{\prime} \mathrm{N}, 115^{\circ} 56^{\prime} \mathrm{E}, 1010 \mathrm{~m}, 10-11 . x .1986$, J. Huisman, $20^{\top}$ (UMSP); Kinabalu National Park, Headquarters area, 1560 m, 9.ix.1983, Hevel \& Steiner, 1 tิ (NMNH); Kinabalu National Park, Headquarters, Sg. Silau-Silau \& Sungai Liwagu, 11.viii.2005, W. Mey, 3 ơ (MNHU); SW Sabah, nr. Long Pa Sia (west), 1050 m, 25.xi-8.xii.1987, Achterberg, $10^{\star}$ (RMNH). 
Chimarra antheae is most similar to C. gyrospina, sp. n., C. phlegyas Malicky, and C. thyestes Malicky in possessing a tergum $\mathrm{X}$ with a slender process at midlength on its dorsal margin, and in the shape of the inferior appendages, each of which is narrow apically and has the basomesal margin produced and concavely cupped. The apex of the basal part of inferior appendage in $C$. antheae is distinctly longer than in either $C$. phlegyas or $C$. thyestes and the ventral apex of the phallobase, while being sclerotized and produced as in C. phlegyas, is not nearly so strongly ventrally curved. It differs from C. gyrospina in that the base of the inferior appendage, in lateral view, is less angular, and in having a single, elongate phallic spine rather than a pair of spines, one of which is helically curved.

Adult. Color (in alcohol) yellowish-brown. Length of forewing: male $4.5-5.3 \mathrm{~mm}$, female $5.7 \mathrm{~mm}$. Forewing venation: stem of Rs curved, with enlarged, sclerotized node at inflection; fork at base of discal cell distinctly thickened, asymmetric, length of discal cell about 2 times width; $m$ crossvein proximal to crossveins $s$ and $r-m$, crossvein $s$ not hyaline; $2 \mathrm{~A}$ vein intersecting 3A vein (apparently forked apically). Postocular parietal sclerite moderately elongate. Maxillary palps relatively short, segment 3 distinctly longer than 2, 3 subequal to 5 . Protarsal claws of male not or very little enlarged, symmetrical.

Male genitalia. Abdominal segment VIII short; tergum about as long as sternum, unmodified, entire, without mesal excavation; sternum VIII without posteroventral projection. Abdominal segment IX very short dorsolaterally, tergum very short dorsally, with pair of short apodemes from anterolateral margin; anteroventral margin in lateral view distinctly produced, rounded; posterior margin straight; ventral process projecting posteriorly, short, acute apically. Preanal appendage setose, short, somewhat flattened. Inferior appendage about as long as tergum $\mathrm{X}$; in lateral view strongly dorsally inflected basoventrally, widest basally, very narrow distally, basoventral margin rounded; in ventral view mesally curved, basomesal margin produced to form broad rounded lobe, its surface concave. Tergum $\mathrm{X}$ with sclerotized lateral lobes and projecting mesal membranous lobe; lateral lobe long, with apex broadly rounded, in lateral view simple, rounded apically, lobe bearing 2 sensilla. Phallobase tubular, with pronounced basodorsal expansion, moderately elongate, ventral apex distinctly sclerotized, distinctly projecting, acute, ventrally deflexed. Endotheca length not discernable (not expanded), with granularly textured region and one prominent, elongate endothecal spine. Phallotremal sclerite complex composed of rod and ring structure, rod short, at midlength with pair of elongate lateral sclerites.

Etymology. Named for Anthea Phillips in recognition of her friendship and support during J. Huisman's research in Sabah.

\section{Chimarra dejongi sp. $\mathbf{n}$.}

Fig. 2

Type material. Holotype: $₫$, Malaysia, Sabah, $60 \mathrm{~km}$ W Lahad Datu, Danum Valley Field Centre, nature trail brooklet, $04^{\circ} 58^{\prime}{ }^{\prime} \mathrm{N}, 117^{\circ} 48^{\prime} \mathrm{E}, 180 \mathrm{~m}$, 9-13.ix.1986, J. Huisman (UMSP000107302) (RMNH).

Paratypes. Malaysia, Sabah, $65 \mathrm{~km} \mathrm{~W}$ Lahad Datu, Sg. Purut camp, $04^{\circ} 57^{\prime} \mathrm{N}, 117^{\circ} 45^{\prime} \mathrm{E}, 200 \mathrm{~m}$,

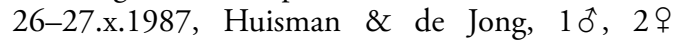
(UMSP); Tawau Hills National Park, Sg. Gelas, 20.viii.2005, W. Mey, 30 (UMHU).

Chimarra dejongi is closely related to and very similar to Chimarra phlegyas Malicky. Both species have nearly identically shaped inferior appendages and internal phallic structure, including the presence of one moderately lengthed phallic spine and two small, tack-like spines distal to the phallotremal sclerite. Chimarra dejongi differs from C. phlegyas primarily in the structure of the ventral apex of the phallobase, which is elongate, strongly sclerotized, and ventrally deflexed in $C$. phlegyas, but scarcely produced and very weakly sclerotized in $C$. dejongi. An additional difference is that the elongate, narrow dorsal processes present on the lateral lobes of tergum $\mathrm{X}$ in C. phlegyas (and also C. antheae, sp. n., C. gyrospina, sp. n., and C. thyestes Malicky) seem to be absent or only minimally developed in $C$. dejongi.

Adult. Color (in alcohol) yellowish-brown. Length of forewing: male $4.2-4.5 \mathrm{~mm}$, female 4.7$4.8 \mathrm{~mm}$. Forewing venation: stem of Rs curved, with enlarged, sclerotized node at inflection; fork at base of discal cell distinctly thickened, asymmetric, length of discal cell about 2 times width; $m$ crossvein proximal to crossveins $s$ and $r-m$, crossvein $s$ not hyaline; $2 \mathrm{~A}$ vein intersecting $3 \mathrm{~A}$ vein (apparently forked apically). Postocular parietal sclerite short. Maxillary palps relatively short, segment 3 slightly longer than 2,3 subequal to 5 . Protarsal claws of male not or very little enlarged, symmetrical.

Male genitalia. Abdominal segment VIII short; tergum longer than sternum, unmodified; sternum VIII without posteroventral projection. Abdominal segment IX short dorsolaterally, tergum short dorsally, with pair of distinct apodemes from anterolateral margin; anteroventral margin in lateral view distinctly produced, rounded; posterior margin 
a

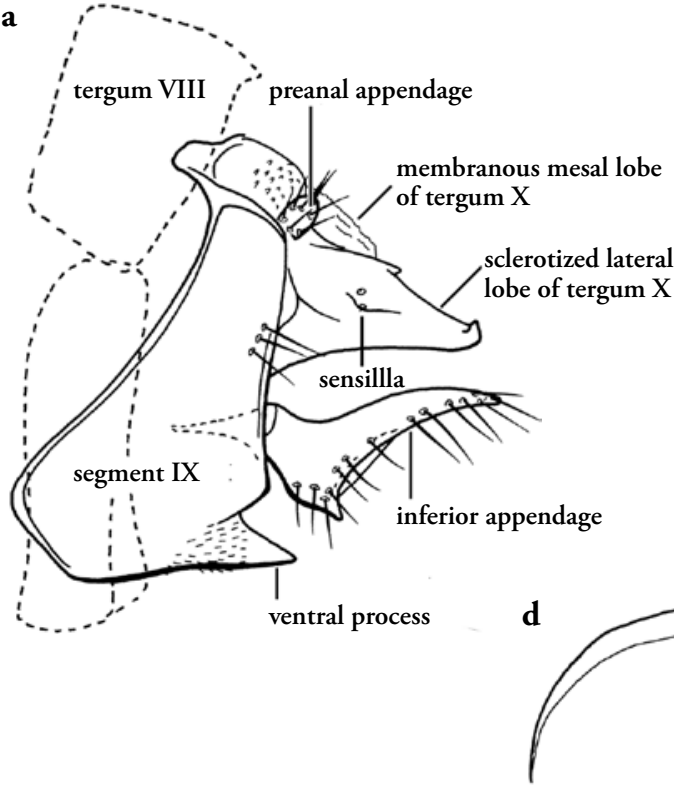

b membranous mesal lobe of tergum X

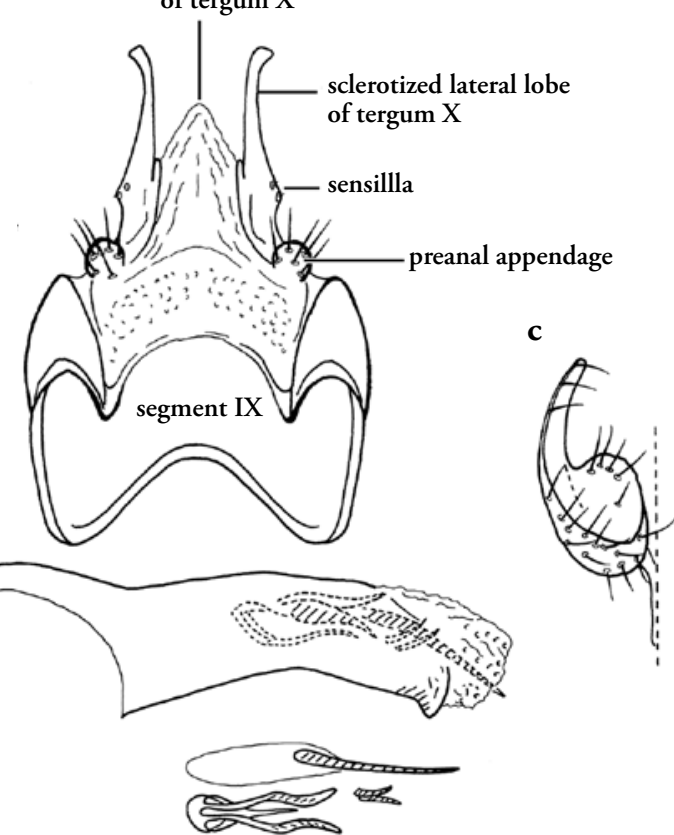

Fig. 2. Chimarra dejongi, male genitalia. - a, lateral; b, segment IX and tergum X, dorsal; c, inferior appendage, ventral; $\mathrm{d}$, phallic apparatus, lateral; inset, dorsal of phallotremal sclerite complex and phallic spine.

straight; ventral process projecting posteriorly, short, acute apically. Preanal appendage setose, short, somewhat flattened. Inferior appendage about as long as tergum $\mathrm{X}$; in lateral view strongly dorsally inflected basoventrally, widest basally, narrowing distally, basoventral margin with subacute projection, apex subacute; in ventral view mesally curved, basomesal margin produced to form broad rounded lobe, its surface concave. Tergum $\mathrm{X}$ with sclerotized lateral lobes and projecting mesal membranous lobe; lateral lobe moderately long, with apex narrowed, subacute, lobe bearing 2 sensilla, sensilla at about midlength, not or scarcely projecting, apex of ventral margin slightly hooked. Phallobase tubular, with pronounced basodorsal expansion, moderately elongate, ventral apex weakly sclerotized, slightly projecting, subacute, weakly ventrally deflexed. Endotheca length not discernable (not expanded), with granularly textured region and 3 endothecal spines, 1 moderately long, probably basal, and 2 very short, probably apical. Phallotremal sclerite complex composed of rod and ring structure, rod short, dorsolaterally, with pair of short curved sclerites.

Etymology. Named in honor of Dr. Rienk de Jong, friend and colleague of J. Huisman at the National Museum of Natural History Naturalis, Leiden.

\section{Chimarra demeter Malicky}

Fig. 3

Chimarra demeter Malicky 2000: 32, holotype ô, Malaysia (Sabah), collection of H. Malicky.

Material examined. Malaysia, Sabah, Danum Valley Field Centre, Sg. Segama, 18.viii.2005, W. Mey, 10 (UMHU); $60 \mathrm{~km} \mathrm{~W} \mathrm{Lahad} \mathrm{Datu,} \mathrm{Danum} \mathrm{Valley} \mathrm{Field} \mathrm{Centre,}$ Sg. Palum Tambun \& vicinity, $04^{\circ} 58^{\prime} \mathrm{N}, 117^{\circ} 48^{\prime} \mathrm{E}, 150 \mathrm{~m}$, 9.ix.1986, J. Huisman, 70, 6 ㅇ (RMNH); same locality, 19-26.iii.1987, J. Huisman, 590 , 59 (UMSP); $60 \mathrm{~km}$ W Lahad Datu, Danum Valley Field Centre, Sg. Palum Tambun, $04^{\circ} 57^{\prime} 30^{\prime} \mathrm{N}, 117^{\circ} 48^{\prime} \mathrm{E}, 180 \mathrm{~m}, 18$. iii. 1987 , J. Huisman, 60, 29 (RMNH); Danum Valley Field Centre, nature trail brooklet, $04^{\circ} 58^{\prime} \mathrm{N}, 117^{\circ} 48^{\prime} \mathrm{E}, 180 \mathrm{~m}$, 9.ix.1986, J. Huisman, 50, 10 (UMSP); Long Pa Sia, airstrip, $04^{\circ} 24^{\prime} \mathrm{N}, 115^{\circ} 43^{\prime} \mathrm{E}, 1000 \mathrm{~m}, 16 . i v .1987$, Huisman \& van Tol, 20 , 7 ㅇ (RMNH); Kg. Long Pa Sia, confluence Sg. Pa Sia \& Sg. Matang, $04^{\circ} 24^{\prime} \mathrm{N}, 115^{\circ} 43^{\prime} \mathrm{E}$, 1000 m, 13.iv.1987, J. Huisman, 20 (UMSP); 60 km W Lahad Dalu, Danum Valley Field Centre, Ulu Segama, $04^{\circ} 58^{\prime} \mathrm{N}, 117^{\circ} 48^{\prime} \mathrm{E}, 150 \mathrm{~m}, 8-13 . i x .1986$, J. Huisman, $20^{\widehat{O}}$ (UMSP); nr. Danum Valley Field Centre, W10, 150 m, 2-23.viii.1987, Achterberg \& Kennedy, $10^{\text {ते }}$ (RMNH); $60 \mathrm{~km} \mathrm{~W}$ Lahad Datu, Danum Valley Field Centre, nr. Segama bridge, $04^{\circ} 58^{\prime} \mathrm{N}, 117^{\circ} 48^{\prime} \mathrm{E}$, 150 m, 20.x.1987, Huisman \& de Jong, 10 (RMNH); $10 \mathrm{~km}$ NNE Ranau, Kg. Randagung baru, Sg. Pangatan, $06^{\circ} 02^{\prime} \mathrm{N}, 116^{\circ} 44^{\prime} \mathrm{E}, 350 \mathrm{~m}, 6 . i i .1987$, J. Huisman, 23 ô, $^{\prime}$ 7 오 (UMSP). 
a

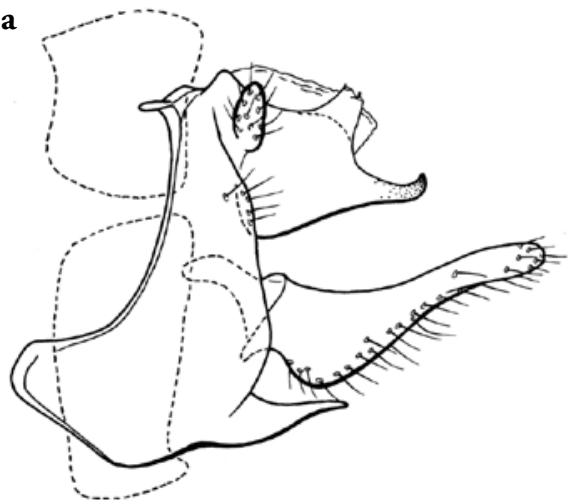

b

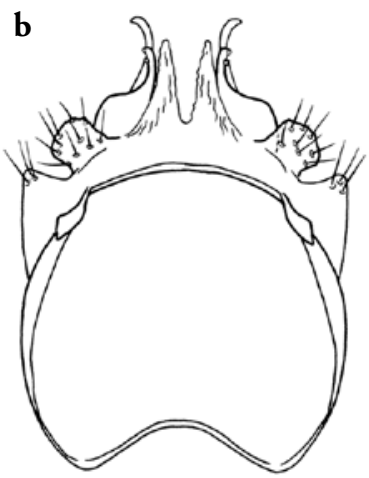

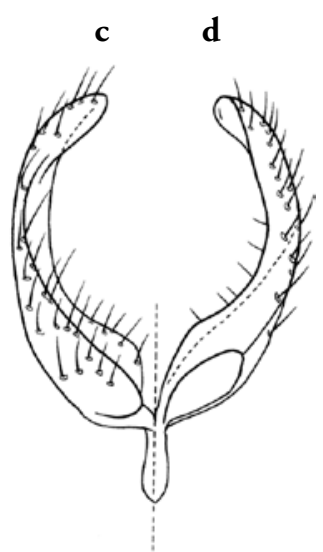

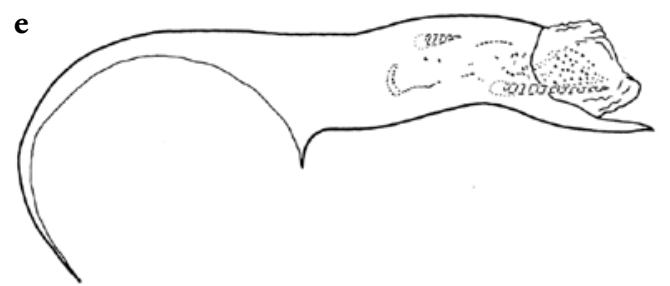

Fig. 3. Chimarra demeter, male genitalia. - a, lateral; b, segment IX and tergum X, dorsal; c, inferior appendage, ventral; $d$, inferior appendage, dorsal; e, phallic apparatus, lateral.

Among species from Borneo, Chimarra demeter is similar to both $C$. devogeli, sp. n. and C. drepane, sp. n. However, the inferior appendages of $C$. demeter are distinctly shorter than either of these species and the ventral process of segment IX is not as narrow basally. The most diagnostic and distinguishing character of C. demeter is the shape of the lateral lobes of tergum $\mathrm{X}$, each of which has a distinctive short, truncate projection on its dorsal margin bearing 2 sensilla and a projecting, attenuate ventral apex that is slightly dorsally hooked.

Adult. Color (in alcohol) brown. Length of forewing: male 4.4-5.2 $\mathrm{mm}$, female 4.6-5.8 $\mathrm{mm}$. Forewing venation: stem of Rs curved, with enlarged, sclerotized node at inflection; fork at base of discal cell distinctly thickened, asymmetric, length of discal cell about 2.5 times width; $m$ crossvein proximal to crossveins $s$ and $r-m$, crossvein $s$ not hyaline; $2 \mathrm{~A}$ vein intersecting 3A vein (apparently forked apically). Postocular parietal sclerite short. Maxillary palps relatively short, segment 3 subequal to 2 and 5. Protarsal claws of male slightly enlarged, symmetrical.

Male genitalia. Abdominal segment VIII short; tergum about as long as sternum, unmodified, entire, without mesal excavation; sternum VIII with indistinct posteroventral projection. Abdominal segment IX short dorsolaterally, tergum very short dorsally, with pair of short apodemes from anterolateral margin; anteroventral margin in lateral view distinctly produced, rounded; posterior margin nearly straight; ventral process projecting posteriorly, elongate, narrowing from base, acute apically. Preanal appendage setose, short, somewhat flattened. Inferior appendage longer than tergum X; in lateral view strongly dorsally inflected basoventrally, widest basally, narrowing distally, basoventral margin rounded, apex rounded; in ventral view mesally curved, mesal surface without processes. Moderately elongate, dorsally inflected from base, uniformly mesally curved; appendage widest basally, nearly uniform in width distally, apices rounded. Tergum $\mathrm{X}$ with sclerotized lateral lobes and projecting mesal membranous lobe; lateral lobe short, with projecting ventral margin, lobe bearing 2 sensilla, sensilla on short, truncate middorsal lobe, apex of ventral margin attenuate, slightly dorsally hooked. Phallobase tubular, with pronounced basodorsal expansion, moderately elongate, ventral margin slightly bulged basally, ventral apex distinctly sclerotized, distinctly projecting, acute. Endotheca length not discernable (not expanded), with granularly textured region and 1 or 2 short endothecal spines. Phallotremal sclerite complex composed of rod and ring structure, rod very short. 

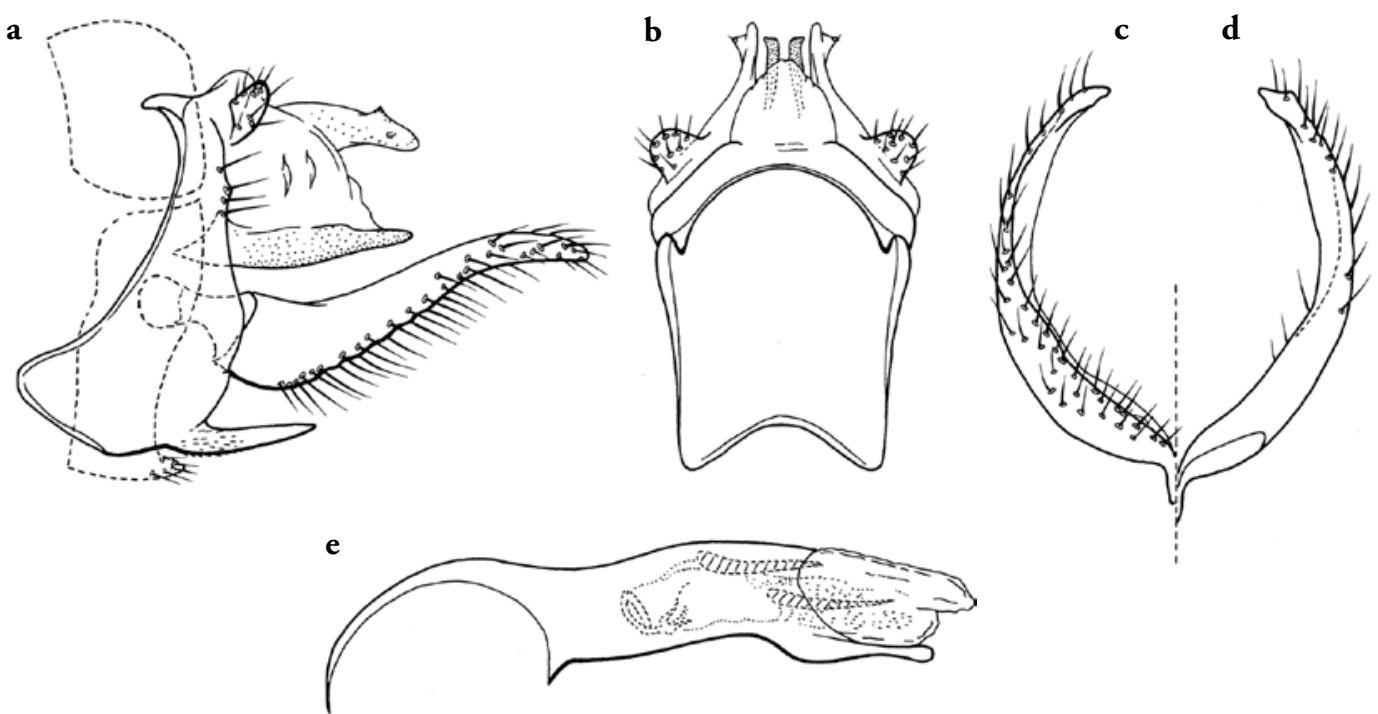

Fig. 4. Chimarra devogeli, male genitalia. - a, lateral; b, segment IX and tergum X, dorsal; c, inferior appendage, ventral; $\mathrm{d}$, inferior appendage, dorsal; e, phallic apparatus, lateral.

\section{Chimarra devogeli sp. $\mathbf{n}$.}

Figs 4, 39

Type material. Holotype: $\delta$, Malaysia, Sabah, Long Pa Sia, confluence Sg. Pa Sia \& Sg. Matang, $04^{\circ} 24^{\prime} \mathrm{N}, 115^{\circ} 43^{\prime} \mathrm{E}, 1000 \mathrm{~m}$, 1.iv.1987, J. Huisman (UMSP000107190) (RMNH).

Paratypes. Malaysia, Sabah, Danum Valley Field Centre, Sungai Kalisun, Sungai Palun Tambun, \& Sungai Segama, 17-19.viii.2005, W. Mey, 240ત, 3 (UMHU); Danum Valley Field Centre, Sungai

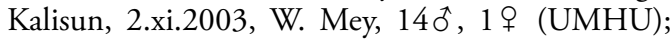
Kinabatangan, bridge, 22.viii.2005, W. Mey, $1 \delta$ (UMHU); Long Pa Sia, Sg. Matang, 04²4'N, 11543'E, 6.xii.1987, Huisman \& Achterberg, 50, 5 ㅇ (UMSP); $60 \mathrm{~km}$ W Lahad Datu, Danum Valley Field Centre, Sg. Palum Tambun $\&$ vicinity, $04^{\circ} 58^{\prime} \mathrm{N}$, 117²8’ E, 150 m, 19-26.iii.1987, J. Huisman, 7 đે, 49 (UMSP); Long Pa Sia, confluence Sg. Pa Sia \& Sg. Matang, $04^{\circ} 24^{\prime} \mathrm{N}, 115^{\circ} 43^{\prime} \mathrm{E}, 1000 \mathrm{~m}$, 1.iv.1987, J. Huisman, 13 đิ (RMNH); same locality, 5.iv.1987, J. Huisman, $3 \hat{0}, 8$ ( $9 M N H)$; Long Pa Sia airstrip, $04^{\circ} 24^{\prime} \mathrm{N}, 115^{\circ} 43^{\prime} \mathrm{E}, 1000 \mathrm{~m}$, 7.xii.1987, J. Huisman, 20, 3 ㅇ (UMSP); $75 \mathrm{~km}$. W Lahad Datu, confl. S. Sabran, S. Danum S/N, 0434'12"N, 117²4’36”E, 200 m, 23.x.1987, J. Huisman, 10, 1 ㅇ (RMNH); Kg. Long Pa Sia, confluence Sg. Pa Sia \& Sg. Matang, $04^{\circ} 24^{\prime} \mathrm{N}, 115^{\circ} 43^{\prime} \mathrm{E}, 1000 \mathrm{~m}$, 13.iv.1987, J. Huisman, $190^{\star}$ (UMSP); $60 \mathrm{~km}$ W Lahad Dalu, Danum Valley Field Centre, Ulu Segama, $04^{\circ} 58^{\prime} \mathrm{N}, 117^{\circ} 48^{\prime} \mathrm{E}, 150$ m, 8-13.ix.1986,
J. Huisman, 10 (RMNH); nr. Danum Valley Field Centre, W9, 210 m, 14-26.iii.1987, Achterberg, 20 , 7 ? (RMNH); Sungai Moyig at tributary, $8 \mathrm{mi}$ E Penampung, 1.x.1978, G. \& C. Edmunds, $7 \hat{0}$ $(\mathrm{NMNH})$.

Chimarra devogeli is most similar to C. tawitawi Malicky from the Philippines, particularly in the overall shape of the inferior appendages and general structure of tergum X. It differs in that the dorsal projection from tergum $\mathrm{X}$ is more elongate, with distinct projecting apical sensilla, and the ventral apex of the phallobase has a distinct, sinuous deflection. Considering the overall similarity of the species, it is likely that the record of $C$. tawitawi from Borneo by Malicky (2008) actually represents this species. Among species from Borneo, Chimarra devogeli is most similar to $C$. drepane, sp. n. and $C$. demeter Malicky. It resembles both those species in having elongate narrow inferior appendages without mesal projections, and particularly in the structure of tergum $\mathrm{X}$, which has the ventral margin forming a sclerous projection and a dorsal margin with a process bearing two sensilla. It differs in that the inferior appendages are more elongate than $C$. demeter, but shorter than in C. drepane, and in the shape of the dorsal process of tergum $\mathrm{X}$, which is not upright and truncate as in $C$. demeter. The slightly decurved and projecting apex of the phallobase seems to be a consistent difference between this species and $C$. drepane. 
Adult. Color (in alcohol) light brown. Length of forewing: male $4.7-5.0 \mathrm{~mm}$, female $4.2-5.9 \mathrm{~mm}$. Forewing venation: stem of Rs curved, with enlarged, sclerotized node at inflection; fork at base of discal cell distinctly thickened, asymmetric, length of discal cell about 2.5 times width; $m$ crossvein proximal to crossveins $s$ and $r-m$, crossvein $s$ not hyaline; 2A vein intersecting 3A vein (apparently forked apically). Postocular parietal sclerite short. Maxillary palps moderately elongate, segment 3 subequal to 2 and 5 . Protarsal claws of male not or very little enlarged, symmetrical.

Male genitalia. Abdominal segment VIII short; tergum about as long as sternum, unmodified; sternum VIII with slight posteroventral projection. Abdominal segment IX very short dorsolaterally, tergum very short dorsally, with pair of distinct apodemes from anterolateral margin; anteroventral margin in lateral view distinctly produced, angular; posterior margin nearly straight; ventral process projecting posteriorly, elongate, narrow, acute apically. Preanal appendage setose, short, rounded, broadest basally. Inferior appendage elongate, much longer than tergum X; in lateral view dorsally inflected basoventrally, widest basally, gradually narrowing distally, basoventral margin rounded, apex subacute; in ventral view uniformly mesally curved, mesal surface without processes. Tergum $\mathrm{X}$ with sclerotized lateral lobes and projecting mesal membranous lobe; lateral lobe short, with projecting ventral margin, lobe bearing 2 sensilla, sensilla on projecting, digitate dorsal lobe, apex of ventral margin narrow, subacute. Phallobase tubular, with pronounced basodorsal expansion, moderately elongate, ventral margin slightly bulged basally, slightly deflexed preapically, ventral apex distinctly sclerotized, distinctly projecting, subacute. Endotheca length not discernable (not expanded), with granularly textured region and 2 short, subequal endothecal spines. Phallotremal sclerite complex composed of rod and ring structure, rod very short, preapically with short lateral sclerites.

Etymology. Named in honor of Dr. Ed de Vogel, Hortus Botanicus, University of Leiden, friend and colleague of J. Huisman.

\section{Chimarra drepane sp. $\mathrm{n}$.}

Fig. 5

Type material. Holotype: $\widehat{o}$, Malaysia, Sabah, 10 km SE Ranau, Kg. Nalapak, Sg. Kananapun, $05^{\circ} 59^{\prime} \mathrm{N}, 116^{\circ} 47^{\prime} \mathrm{E}, 350 \mathrm{~m}, 7 . i i .1987$, J. Huisman, (UMSP000107169) (RMNH).

Paratypes. Malaysia, Sabah, $60 \mathrm{~km}$ W Lahad Datu, Danum Valley Field Centre, Sg. Palum Tambun \& vicinity, $04^{\circ} 58^{\prime} \mathrm{N}, 117^{\circ} 48^{\prime} \mathrm{E}, 150 \mathrm{~m}, 19-26 . i i i .1987$, J. Huisman, 1 ô (RMNH); Sapulut, kampong, roadside, $04^{\circ} 42^{\prime} \mathrm{N}, 116^{\circ} 29^{\prime} \mathrm{E}, 290 \mathrm{~m}$, 4.v.1987, J. Huisman, 10,1 ( $\mathrm{O}$ (UMSP).

Chimarra drepane is probably most similar to C. monorum Chantaramongkol \& Malicky, described from Thailand, particularly in the length and shape of the inferior appendages, but differs in details of tergum X. Among species from Borneo, C. drepane is similar to $C$ devogeli, sp. n. and $C$. demeter Malicky. It resembles those species in having elongate narrow inferior appendages without mesal projections, and particularly in the structure of tergum X, which has the ventral margin forming a sclerous projection and a dorsal margin with a process bearing two sensilla. It differs in that the inferior appendages are more elongate than $C$. devogeli, and much more elongate than $C$. demeter, and in the shape of the dorsal process of tergum $X$, which is not upright and truncate as in $C$. demeter. The shorter phallobase, with a nearly straight projecting ventral apex seems to be a consistent difference between $C$. drepane and $C$. devogeli, which it otherwise closely resembles.

Adult. Color (in alcohol) light brown. Length of forewing: male $3.8-4.6 \mathrm{~mm}$, female $4.5-4.7 \mathrm{~mm}$. Forewing venation: stem of Rs curved, with enlarged, sclerotized node at inflection; fork at base of discal cell distinctly thickened, asymmetric, length of discal cell about 2 times width; $m$ crossvein proximal to crossveins $s$ and $r-m$, crossvein $s$ not hyaline; 2A vein intersecting 3A vein (apparently forked apically). Postocular parietal sclerite short. Maxillary palps moderately elongate, segment 3 subequal to 2 and 5. Protarsal claws of male not or very little enlarged, symmetrical.

Male genitalia. Abdominal segment VIII short; tergum about as long as sternum, unmodified; sternum VIII with slight posteroventral projection. Abdominal segment IX very short dorsolaterally, tergum very short dorsally, with pair of short apodemes from anterolateral margin; anteroventral margin in lateral view distinctly produced, angular; posterior margin nearly straight; ventral process projecting posteriorly, elongate, narrow, acute apically. Preanal appendage setose, short, somewhat flattened. Inferior appendage very elongate, much longer than tergum X; in lateral view strongly dorsally inflected basoventrally, nearly uniformly wide, basoventral margin rounded, apex rounded; in ventral view uniformly mesally curved, mesal surface without processes. Tergum $\mathrm{X}$ with sclerotized lateral lobes and projecting mesal membranous lobe; in lateral view with projecting ventral margin, apex of ventral margin narrow, subacute; dorsal margin with projecting, rounded lobe with 

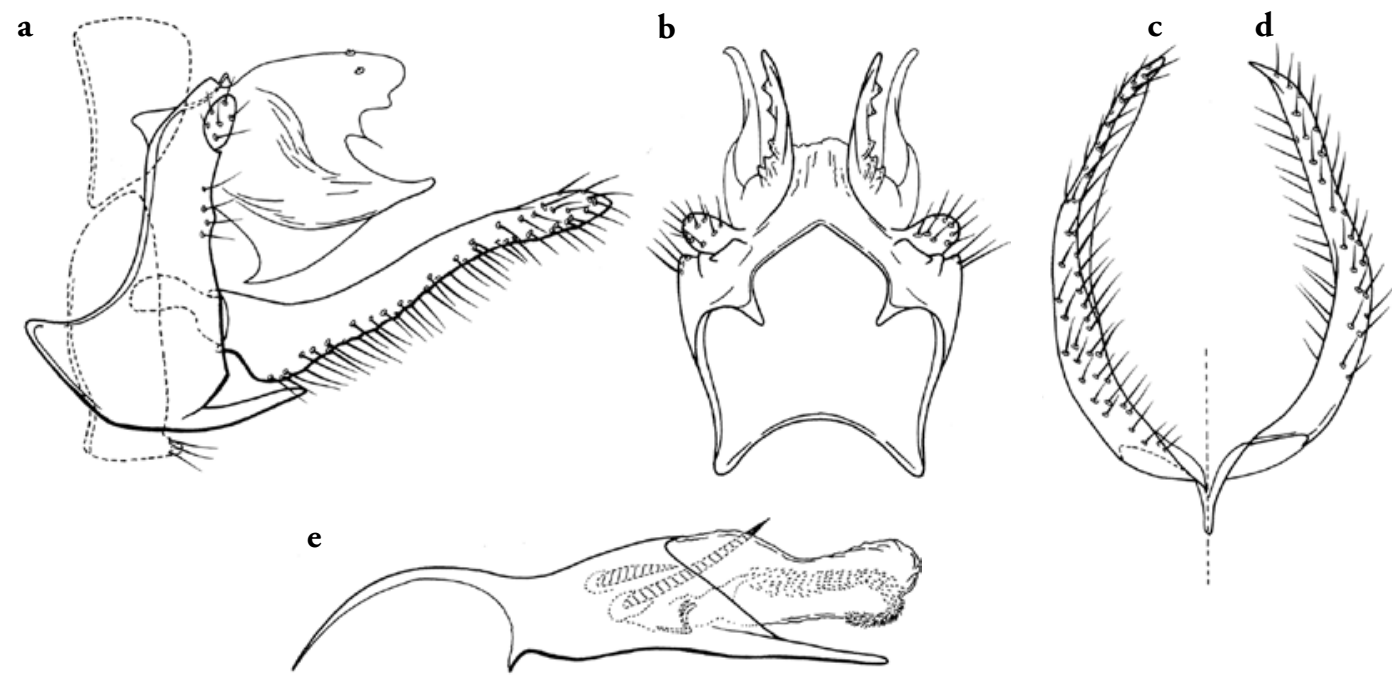

Fig. 5. Chimarra drepane, male genitalia. - a, lateral; b, segment IX and tergum X, dorsal; c, inferior appendage, ventral; $\mathrm{d}$, inferior appendage, dorsal; e, phallic apparatus, lateral.

2 sensilla. Phallobase tubular, with pronounced basodorsal expansion, relatively short, slightly expanded apically, ventral margin slightly bulged basally, ventral apex distinctly sclerotized, distinctly projecting, acute. Endotheca length not discernable (not expanded), with apparent tract of numerous minute spines and 2 prominent endothecal spines, 1 moderate in length and 1 very short. Phallotremal sclerite complex composed of rod and ring structure, rod very short, preapically with short lateral sclerites.

Etymology. This species is named Chimarra drepane from the Greek word drepane, a sickle, used as a noun in apposition, and referring to the elongate, mesally curved and sickle-shaped inferior appendages.

\section{Chimarra dulitensis Kimmins}

Fig. 6

Chimarra dulitensis Kimmins, 1955: 378, holotype đo , Malaysia (Sarawak), BMNH; Malicky 1989: 135, Indonesia (Sumatra).

Material examined. Malaysia, Sabah, SE Sabah nr. Danum Valley Field Center, 240 m, 18.x-22.xi.1987, Achterberg, 10 (RMNH); Long Pa Sia, confluence Sg. Pa Sia \& Sg. Matang, $04^{\circ} 24^{\prime} \mathrm{N}, 115^{\circ} 43^{\prime} \mathrm{E}, 1000 \mathrm{~m}, 10 . i v .1987$, J. Huisman, $10^{\widehat{t}}$ (UMSP); $60 \mathrm{~km}$ W Lahad Datu, Danum Valley Field Centre, nr. Segama bridge, $04^{\circ} 58^{\prime} \mathrm{N}, 117^{\circ} 48^{\prime} \mathrm{E}$, 150 m, 20.x.1987, Huisman \& de Jong, 10 (UMSP).

Chimarra dulitensis is a distinctive member of the Chimarra digitata lineage. Among species from
Borneo, it is easily recognized by the position of the sensilla of tergum $\mathrm{X}$, which are on a rounded process near its ventral margin, and by the general shape of the inferior appendages, which also have a small mesal projection. The single, elongate phallic spine is also a distinctive character, although possibly variable. It is elongate in the holotype illustration by Kimmins and also in the specimens from Sabah, but less elongate in the specimen illustrated by Malicky (1989, fig. 8a-c) from Sumatra.

Adult. Color (in alcohol) light brown. Length of forewing: male $4.2-4.5 \mathrm{~mm}$. Forewing venation: stem of Rs curved, with enlarged, sclerotized node at inflection; fork at base of discal cell distinctly thickened, asymmetric, length of discal cell about 2 times width; $m$ crossvein proximal to crossveins $s$ and $r-m$, crossvein $s$ not hyaline; $2 \mathrm{~A}$ vein intersecting $3 \mathrm{~A}$ vein (apparently forked apically). Postocular parietal sclerite short. Maxillary palps relatively short, segment 3 subequal to 2 and 5. Protarsal claws of male not or very little enlarged, symmetrical.

Male genitalia. Abdominal segment VIII short; tergum longer than sternum, unmodified; sternum VIII without posteroventral projection. Abdominal segment IX short dorsolaterally, tergum short dorsally, with pair of short apodemes from anterolateral margin; anteroventral margin in lateral view produced, angular; posterior margin nearly straight; ventral process projecting posteriorly, moderately elongate, narrowing from base, acute apically. Preanal appendage setose, short, somewhat flattened. Inferior appendage longer than tergum X; in lateral 

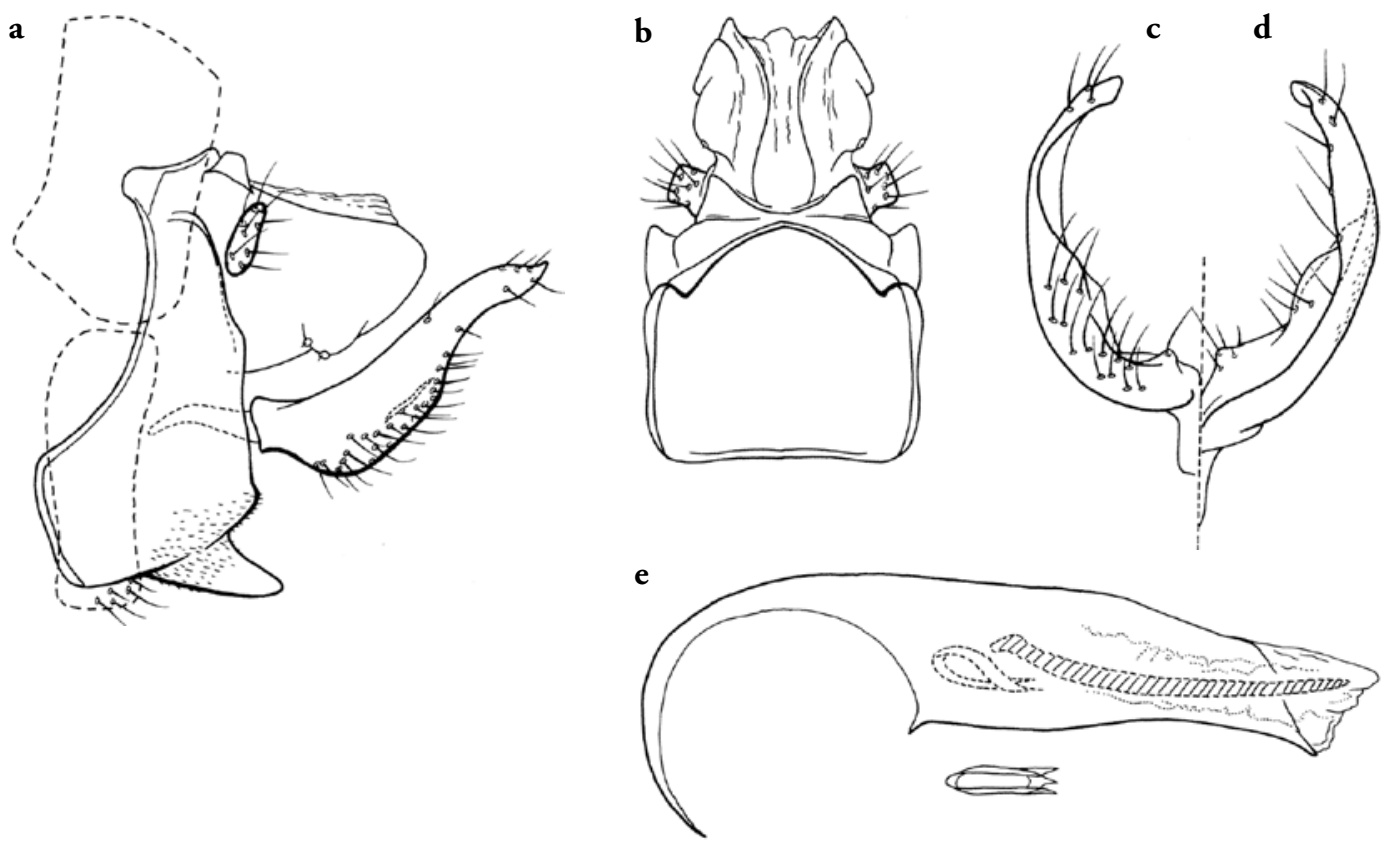

Fig. 6. Chimarra dulitensis, male genitalia. - a, lateral; b, segment IX and tergum X, dorsal; c, inferior appendage, ventral; $\mathrm{d}$, inferior appendage, dorsal; e, phallic apparatus, lateral.

view dorsally inflected basoventrally, widest basally, narrowing distally, basoventral margin rounded, apex rounded; in ventral view mesally curved, mesal surface with indistinct, broadly rounded projection at midlength. Tergum $\mathrm{X}$ with sclerotized lateral lobes and projecting mesal membranous lobe; with apex broadly rounded, lobe bearing 2 sensilla, sensilla on slightly projecting, ventrolateral lobe. Phallobase tubular, with pronounced basodorsal expansion, moderately elongate, ventral margin slightly bulged basally, ventral apex weakly sclerotized, slightly projecting. Endotheca length not discernable (not expanded), granularly textured region not discernable, with 1, prominent, elongate spine. Phallotremal sclerite complex composed of elongate ring, rod very reduced.

\section{Chimarra fuilianae sp. $\mathbf{n}$.}

Fig. 7

Type material. Holotype: $\widehat{\jmath}$, Malaysia, Sabah, $12 \mathrm{~km}$ NNE Ranau, Poring Hot Spring, Sg. Kepungit, $06^{\circ} 03^{\prime} \mathrm{N}, 116^{\circ} 42^{\prime} \mathrm{E}, 550 \mathrm{~m}$, 24.i.1987, J. Huisman (UMSP000107250) (RMNH).

Paratypes. Malaysia, Sarawak, Gunung Gading National Park, Sungai Lundu, 23-26.x.2003, W. Mey, 50 (UMHU); Sabah, 9.5 km NNW Kundassang,
Marei-Parei, Sg. Tawubang, $06^{\circ} 04^{\prime} \mathrm{N}, 116^{\circ} 30^{\prime} \mathrm{E}$, 1030 m, 11.iii.1987, J. Huisman, $20^{\Uparrow}$ (UMSP).

Chimarra fuilianae is similar to C. tityos Malicky and C. terramater Malicky, but possibly most closely related to C. xiphosella. All of these species have the character combination of a tergum $X$ with a projecting and somewhat upturned ventral margin, sensilla on a rounded projection at midlength on the same structure, an inferior appendage with a projection from its mesal margin, and a relatively elongate ventral process on segment IX. Among these species, C. fuilianae is distinguished by the relatively narrow and rounded basal margin of its inferior appendage, as well as the overall shape of tergum $\mathrm{X}$ and the shape and length of the ventral process of segment IX.

Adult. Color (in alcohol) yellowish-brown. Length of forewing: male $3.8-4.1 \mathrm{~mm}$. Forewing venation: stem of Rs curved, with enlarged, sclerotized node at inflection; fork at base of discal cell distinctly thickened, asymmetric, length of discal cell about 2 times width; $m$ crossvein proximal to crossveins $s$ and $r-m$, crossvein $s$ not hyaline; $2 \mathrm{~A}$ vein intersecting $3 \mathrm{~A}$ vein (apparently forked apically). Postocular parietal sclerite short. Maxillary palps moderately elongate, segment 3 distinctly longer than 2, 3 subequal to 5 . Protarsal claws of male not or very little enlarged, symmetrical. 

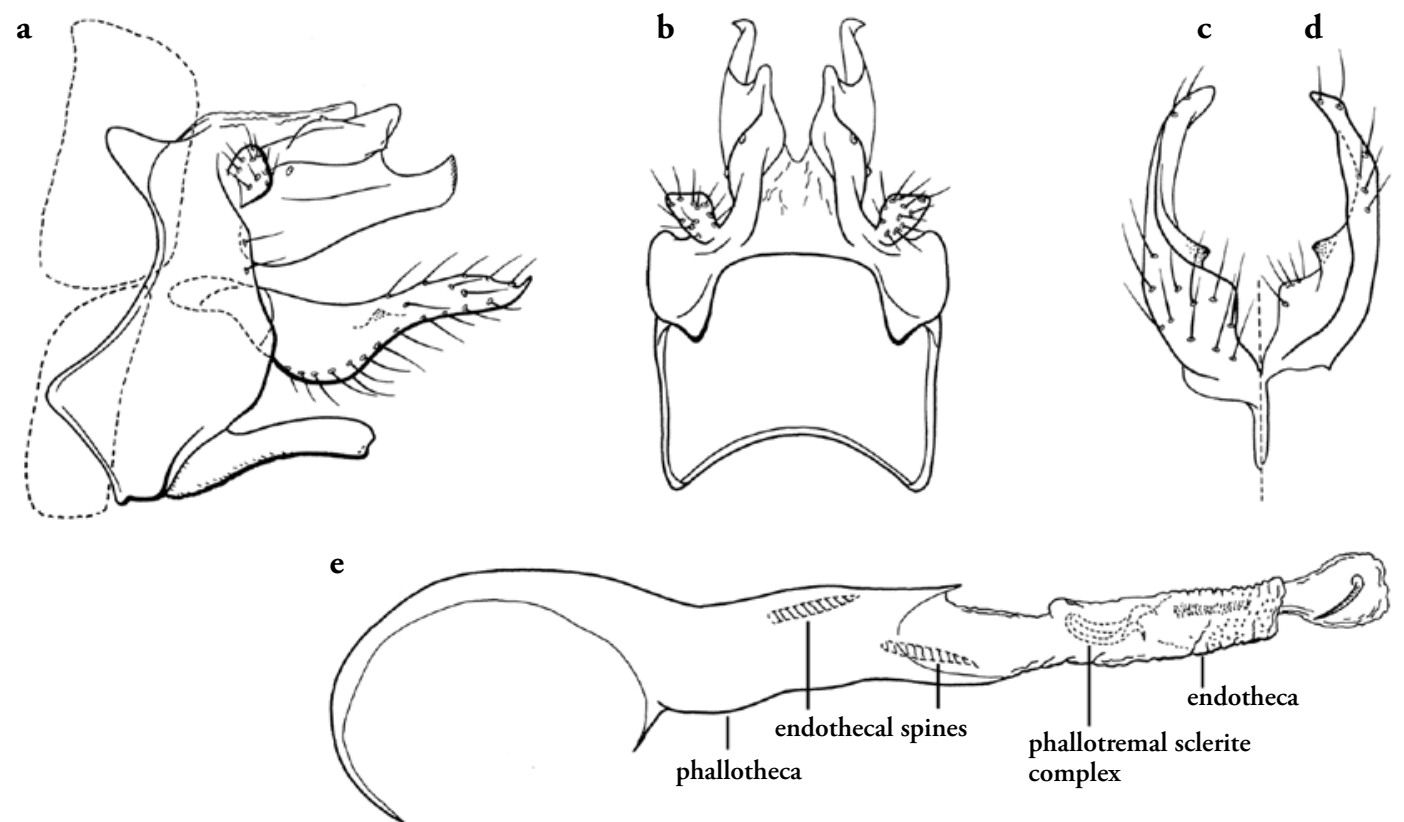

Fig. 7. Chimarra fuilianae, male genitalia. - a, lateral; b, segment IX and tergum X, dorsal; c, inferior appendage, ventral; $\mathrm{d}$, inferior appendage, dorsal; e, phallic apparatus, lateral.

Male genitalia. Abdominal segment VIII short; tergum about as long as sternum, unmodified; sternum VIII without posteroventral projection. Abdominal segment IX short dorsolaterally, tergum short dorsally, with pair of distinct apodemes from anterolateral margin; anteroventral margin in lateral view distinctly produced, angular; posterior margin slightly produced; ventral process projecting posteriorly, elongate, nearly uniform in width, rounded apically. Preanal appendage setose, short, rounded or subquadrate, somewhat flattened, slightly narrowed basally. Inferior appendage longer than tergum $\mathrm{X}$; in lateral view dorsally inflected basoventrally, widest basally, narrowing distally, basoventral margin rounded, apex subacute; in ventral view mesally curved, basomesally with slightly angulate projection, mesal surface with tooth-like projection at midlength. Tergum $\mathrm{X}$ with sclerotized lateral lobes and projecting mesal membranous lobe; lateral lobe with projecting ventral margin, lobe bearing 2 sensilla, sensilla on slightly projecting, rounded middorsal lobe, apex of ventral margin relatively broad, slightly hooked. Phallobase tubular, with pronounced basodorsal expansion, moderately elongate, ventral margin slightly bulged basally, ventral apex weakly sclerotized, slightly projecting, acute. Endotheca elongate, tubular, with granularly textured region; 3 endothecal spines, 2 subequal, short, basal spines and 1 short, slender, curved apical spine. Phallotremal sclerite complex composed of elongate ring, rod not evident, apically with pair of short, curved, lateral sclerites.

Etymology. Named in honor of Fui-Lian Tan, friend of J. Huisman.

\section{Chimarra gyrospina sp. $\mathbf{n}$.}

Fig. 8

Type material. Holotype: đ, Malaysia, Sabah, $1 / 2$ way, road Melligan to Long Pa Sia, $04^{\circ} 35^{\prime} \mathrm{N}$, 115²'', 1200 m, 15.xii.1986, J. Huisman (UMSP000107290) (RMNH).

Paratypes. Malaysia, Sabah, same data as holotype, 1 을 (RMNH).

Chimarra gyrospina is similar to C. antheae, sp. n., C. thyestes Malicky, and C. phlegyas Malicky in possessing a tergum $\mathrm{X}$ with a slender process at midlength on its dorsal margin, and in the shape of the inferior appendages, which are very narrow apically and have the basomesal margin produced and concavely cupped. It is easily distinguished by the overall shape of the inferior appendages and differs from the other species mentioned in that the apical phallic spine is very distinctly curled, the ventral apex of the phallobase is less developed, and one of the sensilla 
a

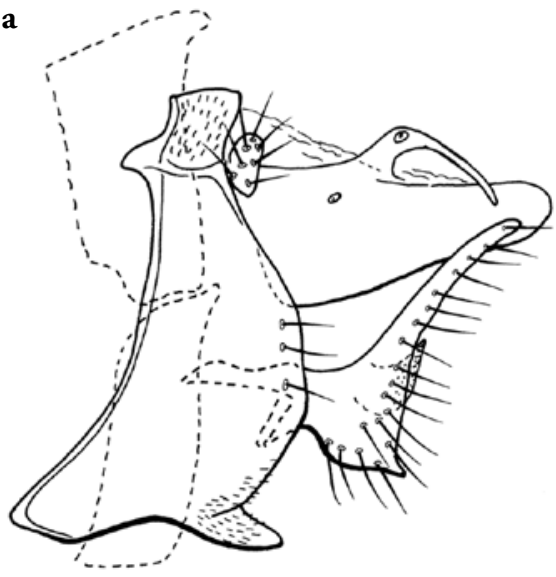

b

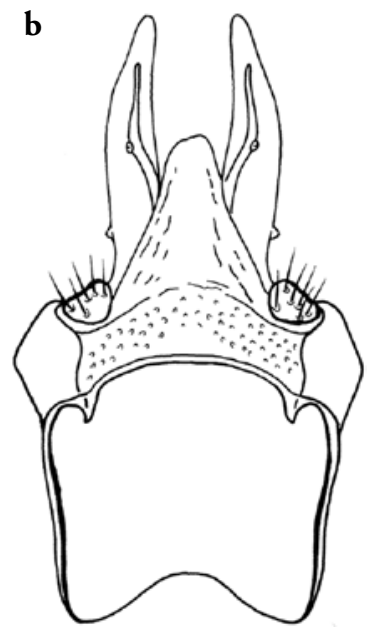

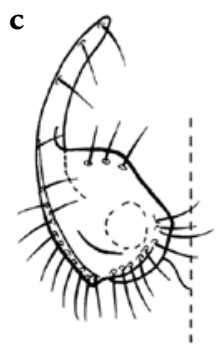

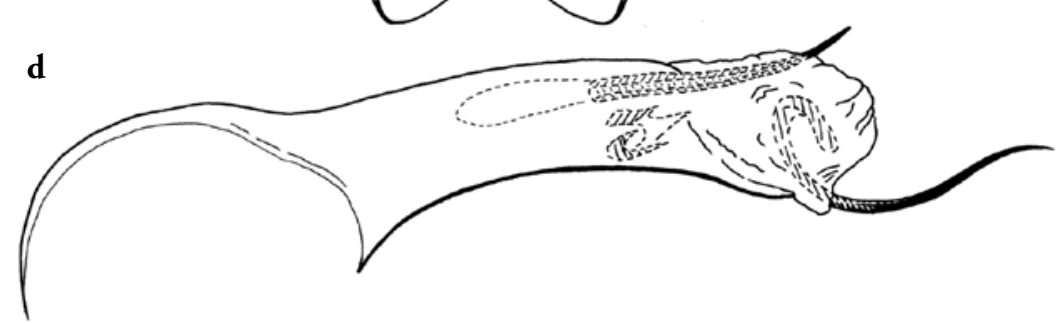

Fig. 8. Chimarra gyrospina, male genitalia. - a, lateral; b, segment IX and tergum X, dorsal; c, inferior appendage, ventral; $\mathrm{d}$, phallic apparatus, lateral.

of tergum X is located on its dorsal process.

Adult. Color (in alcohol) yellowish-brown. Length of forewing: male $4.5 \mathrm{~mm}$, female $5.3 \mathrm{~mm}$. Forewing venation: stem of Rs curved, with enlarged, sclerotized node at inflection; fork at base of discal cell distinctly thickened, asymmetric, length of discal cell about 2.5 times width; $m$ crossvein proximal to crossveins $s$ and $r-m$, crossvein $s$ not hyaline; $2 \mathrm{~A}$ vein intersecting 3A vein (apparently forked apically). Postocular parietal sclerite short. Maxillary palps moderately elongate, segment 3 distinctly longer than 2, 3 subequal to 5. Protarsal claws of male not or very little enlarged, symmetrical.

Male genitalia. Abdominal segment VIII short; tergum about as long as sternum, unmodified; sternum VIII without posteroventral projection. Abdominal segment IX short dorsolaterally, tergum short dorsally, with pair of short apodemes from anterolateral margin; anteroventral margin in lateral view slightly produced, angular; posterior margin slightly produced; ventral process projecting posteriorly, short, narrowing from base, acute apically. Preanal appendage setose, short, somewhat flattened. Inferior appendage about as long as tergum $\mathrm{X}$; in lateral view strongly dorsally inflected basoventrally, widest basally, very narrow distally, basoventral margin acute, apex subacute; in ventral view mesally curved, basomesal margin produced to form broad rounded lobe, its surface concave. Tergum $\mathrm{X}$ with sclerotized lateral lobes and projecting mesal membranous lobe; lateral lobe long, with apex broadly rounded, in lateral view with elongate, digitate process at midlength on dorsal margin, lobe bearing 2 sensilla, one sensillum located basolaterally, other on elongate dorsal process. Phallobase tubular, with pronounced basodorsal expansion, moderately elongate, ventral apex weakly sclerotized, slightly projecting, acute. Endotheca length not discernable (not expanded), granularly textured region not discernable, with 2 endothecal spines, 1 long basal spine and 1 long, slender, curved apical spine. Phallotremal sclerite complex composed of rod and ring structure, rod very short.

Etymology. This species is named Chimarra gyrospina from the Latin word gyrus, meaning to turn around or circle, and referring to the helically bent apical phallic spine. 

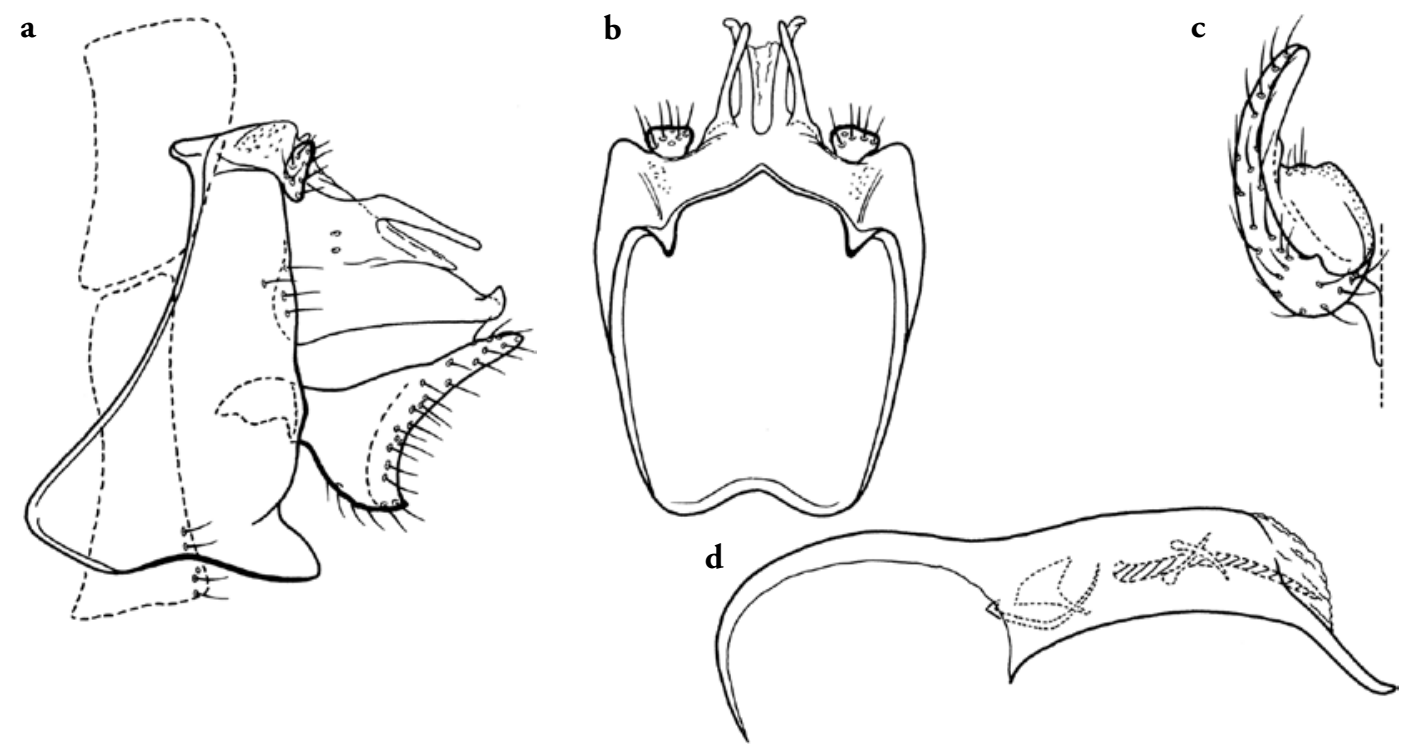

Fig. 9. Chimarra phlegyas, male genitalia. - a, lateral; b, segment IX and tergum X, dorsal; c, inferior appendage, ventral; $\mathrm{d}$, phallic apparatus, lateral.

\section{Chimarra phlegyas Malicky}

Figs 9, 40

Chimarra phlegyas Malicky, 2008: 840, holotype ô, Indonesia (Eastern Kalimantan), Zoologische Museum Lausanne.

Material examined. Malaysia, Sabah, $11 \mathrm{~km} S$ Nabawan, pond on roadside, $04^{\circ} 57^{\prime} \mathrm{N}, 116^{\circ} 27^{\prime} \mathrm{E}, 400 \mathrm{~m}, 15 . x \mathrm{i} .1987$, J. Huisman, 10 (RMNH); Danum Valley Field Center,

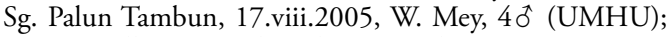
Tawau Hills National Park, Sg. Gelas, 20.viii.2005 \& 3.iii.2006, W. Mey, 8 ô (UMHU); Kinabalu National Park, Poring Hot Springs, 30.x.2003, W. Mey, 10े, (UMHU); $60 \mathrm{~km}$ W of Lahad Datu, DVFC, brooklet above lotus pond, $04^{\circ} 58^{\prime} \mathrm{N}, 117^{\circ} 48^{\prime} \mathrm{E}, 150 \mathrm{~m}, 19-28 . x .1987$, Huisman \& de Jong, 460ิ, 26 (UMSP); $60 \mathrm{~km}$ W Lahad Datu, Danum Valley Field Centre, Sg. Palum Tambun $\&$ vicinity, $04^{\circ} 58^{\prime} \mathrm{N}, 117^{\circ} 48^{\prime} \mathrm{E}, 150 \mathrm{~m}, 19-26 . i i i .1987$, J. Huisman, $50^{\circ}$ (RMNH); Long Pa Sia airstrip, $04^{\circ} 24^{\prime} \mathrm{N}, 115^{\circ} 43^{\prime} \mathrm{E}$, 1000 m, 15.iv.1987, Huisman \& van Tol, 20 (RMNH); Poring Hot Spring, $11 \mathrm{~km}$ NNE Ranau, Sg. Montokungon crossing road, $06^{\circ} 02^{\prime} 30^{\prime \prime} \mathrm{N}, 116^{\circ} 43^{\prime} \mathrm{E}, 450 \mathrm{~m}$, 28.i.1987, J. Huisman, $1 \delta^{\widehat{\sigma}}$ (UMSP); 20 km NNE Ranau, Kg. Takutan, Sg. Mokodou, $06^{\circ} 06^{\prime} \mathrm{N}, 116^{\circ} 45^{\prime} \mathrm{E}, 325$ m, 4.ii.1987; J. Huisman, 10 (RMNH); 12 km NNE Ranau, Poring Hot Spring, staff quarters, $06^{\circ} 03^{\prime} \mathrm{N}, 116^{\circ} 42^{\prime} \mathrm{E}, 550 \mathrm{~m}$, 1.ii.1987, Huisman \& de Jong, 250 (UMSP); $60 \mathrm{~km} \mathrm{~W}$ Lahad Dalu, Danum Valley Field Centre, Ulu Segama, $04^{\circ} 58^{\prime} \mathrm{N}, 117^{\circ} 48^{\prime} \mathrm{E}, 150 \mathrm{~m}, 8-13 . i x .1986$, J. Huisman, $30^{\widehat{0}}$ (RMNH); $60 \mathrm{~km}$ W Lahad Datu, Danum Valley Field Centre, nr. Segama bridge, $04^{\circ} 58^{\prime} \mathrm{N}, 117^{\circ} 48^{\prime} \mathrm{E}, 150 \mathrm{~m}$,

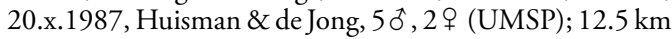
$\mathrm{S}$ Nabawan, on road to $\mathrm{Kg}$. Pamuntariah, 1st bridge, $04^{\circ} 57^{\prime} \mathrm{N}, 116^{\circ} 27^{\prime} \mathrm{E}, 400 \mathrm{~m}, 16 . x \mathrm{i} .1987$, J. Huisman, $10^{\top}$ $(\mathrm{RMNH}) ; 39 \mathrm{~km}$ on road Keningau-Nabawan, Kg. Sook, $05^{\circ} 12^{\prime} \mathrm{N}, 116^{\circ} 19^{\prime} \mathrm{E}, 350 \mathrm{~m}, 17 . x i .1987$, Huisman $\&$ de
Jong, $10,19(\mathrm{RMNH}) ; 60 \mathrm{~km}$ W Lahad Datu, Danum Valley Field Centre, EO trail, Sg. Segama, $04^{\circ} 58^{\prime} \mathrm{N}$, $117^{\circ} 48^{\prime} \mathrm{E}, 150 \mathrm{~m}, 18 . x .1987$, Huisman \& de Jong, $2 \hat{O}^{\text {, }}$ 1 ( RMNH); Sg. Moyig at tributary, 8 mi E Penampung, 1.x.1978, G. \& C. Edmunds, 10 (NMNH).

Chimarra phlegyas is very similar to $C$. dejongi, sp. n. Both species have nearly identically shaped inferior appendages and internal phallic structure, including the presence of one moderately lengthed phallic spine and two small, tack-like spines distal to the phallotremal sclerite. Chimarra phlegyas differs from C. dejongi primarily in the structure of the ventral apex of the phallobase, which is elongate, strongly sclerotized, and ventrally deflexed in C. phlegyas, but scarcely produced and very weakly sclerotized in C. dejongi. An additional difference is that the elongate, narrow dorsal process present on the lateral lobe of tergum $\mathrm{X}$ in C. phlegyas seems to be absent or only minimally developed in $C$. dejongi.

Adult. Color (in alcohol) yellowish-brown. Length of forewing: male 4.0-5.0 mm, female $4.2-5.2 \mathrm{~mm}$. Forewing venation: stem of Rs curved, with enlarged, sclerotized node at inflection; fork at base of discal cell distinctly thickened, asymmetric, length of discal cell about 2.5 times width; $m$ crossvein proximal to crossveins $s$ and $r-m$, crossvein $s$ not hyaline; $2 \mathrm{~A}$ vein intersecting $3 \mathrm{~A}$ vein (apparently forked apically). Postocular parietal sclerite short. Maxillary palps relatively short, segment 3 slightly longer than 2, 3 subequal to 5 . Protarsal claws of male not or very little enlarged, symmetrical. 
Male genitalia. Abdominal segment VIII short; tergum about as long as sternum, unmodified; sternum VIII without posteroventral projection. Abdominal segment IX short dorsolaterally, tergum short dorsally, with pair of distinct apodemes from anterolateral margin; anteroventral margin in lateral view distinctly produced, rounded; posterior margin nearly straight; ventral process projecting posteriorly, short, broad basally, subtriangular. Preanal appendage setose, short, somewhat flattened. Inferior appendage about as long as tergum $\mathrm{X}$; in lateral view strongly dorsally inflected basoventrally, widest basally, gradually narrowing distally, basoventral margin with subacute projection, apex rounded; in ventral view mesally curved, basomesal margin produced to form broad rounded lobe, its surface concave. Tergum X with sclerotized lateral lobes and projecting mesal membranous lobe; lateral lobe moderately long, with projecting ventral margin, in lateral view with elongate, digitate process at midlength on dorsal margin, lobe bearing 2 sensilla, sensilla located basolaterally, apex of ventral margin slightly hooked. Phallobase tubular, with pronounced basodorsal expansion, relatively short, ventral apex distinctly sclerotized, distinctly projecting, acute, strongly ventrally deflexed. Endotheca length not discernable (not expanded), granularly textured region not discernable, with 3 endothecal spines, 1 moderately long, probably basal, and 2 very short, probably apical. Phallotremal sclerite complex composed of rod and ring structure, rod short, at midlength with pair of sclerites.

\section{Chimarra terramater Malicky}

Fig. 10

Chimarra terramater Malicky, 2008: 841, holotype $\widehat{\sigma}$, Indonesia (Eastern Kalimantan), Zoologische Museum Lausanne.

Material examined. Malaysia, Sarawak, Gunung Gading National Park, Sg. Lundu, 23-26.x.2003, W. Mey, $72{ }^{\star}$ (UMHU); Sabah, Poring Hot Spring, $12 \mathrm{~km} \mathrm{NNE}$ Ranau, Sg. Montokungon, $06^{\circ} 02^{\prime} \mathrm{N}, 116^{\circ} 42^{\prime} \mathrm{E}, 525 \mathrm{~m}$, 30.i.1987, J. Huisman,, 1 t , 12 우 (RMNH); Crocker Range National Park, Sg. Myoog, 9.xi.2003, W. Mey, $20^{\star}$ (UMHU); West Coast Zone, Sg. Kepungit waterfall, $06^{\circ} 03^{\prime} \mathrm{N}, 116^{\circ} 42^{\prime} \mathrm{E}, 550 \mathrm{~m}$, 5.xii.1986, J. Huisman, 40 , 1 ㅇ (UMSP); Long Pa Sia, confluence Sg. Pa Sia \& Sg. Matang, $04^{\circ} 24^{\prime} \mathrm{N}, 115^{\circ} 43^{\prime} \mathrm{E}, 1000 \mathrm{~m}, 10$. iv. 1987 , J. Huisman, 10 (UMSP); $3.5 \mathrm{~km} \mathrm{SW} \mathrm{Kg.} \mathrm{Long} \mathrm{Pa} \mathrm{Sia}$ \& Sg. Ritan, $04^{\circ} 24^{\prime} \mathrm{N}, 115^{\circ} 42^{\prime} \mathrm{E}, 1160 \mathrm{~m}, 8-9$. iv.1987, J. Huisman, 5 đ (UMSP); same locality, 14-16.xii.1989, J. Huisman, 3 ㅇ (RMNH); Poring Hot Spring, $11 \mathrm{~km} \mathrm{NNE}$ Ranau, Sg. Montokungon crossing road, $06^{\circ} 02^{\prime} 30^{\prime \prime N}$, $116^{\circ} 43^{\prime} \mathrm{E}, 450 \mathrm{~m}, 28 . i .1987$, J. Huisman, $10^{\dagger}$ (RMNH); $20 \mathrm{~km}$ NNE Ranau, Kg. Takutan, Sg. Mokodou, $06^{\circ} 06^{\prime} \mathrm{N}$, $116^{\circ} 45^{\prime} \mathrm{E}, 325$ m, 4.ii.1987, J. Huisman, 1 ô (RMNH);
Poring Hot Spring, $12 \mathrm{~km}$ NNE Ranau, Sg. Kepungit, E park boundary, $06^{\circ} 03^{\prime} \mathrm{N}, 116^{\circ} 42^{\prime} \mathrm{E}, 480 \mathrm{~m}$, 26.xi.1986, J. Huisman, $30^{\circ}$ (UMSP); Long Pa Sia, airstrip, $04^{\circ} 24^{\prime} \mathrm{N}$, $115^{\circ} 43^{\prime} \mathrm{E}, 1000 \mathrm{~m}$, 16.iv.1987, Huisman \& van Tol, $10^{\top}$ (RMNH); $12 \mathrm{~km}$ NNE Ranau, Poring Hot Spring, staff quarters, $06^{\circ} 03^{\prime} \mathrm{N}, 116^{\circ} 42^{\prime} \mathrm{E}, 550 \mathrm{~m}, 1$. ii. 1987 , Huisman \& de Jong, $1 \delta^{\star}$ (UMSP); 12 km NNE Ranau, Poring Hot Spring, Sg. Tananasad, $06^{\circ} 03^{\prime} \mathrm{N}, 116^{\circ} 42^{\prime} \mathrm{E}$, 560 m, 31.i.1987, J. Huisman, $30^{\wedge}$ (UMSP); Kg. Long $\mathrm{Pa}$ Sia, confluence Sg. Pa Sia \& Sg. Matang, $04^{\circ} 24^{\prime} \mathrm{N}$, $115^{\circ} 43^{\prime} \mathrm{E}, 1000 \mathrm{~m}, 13$.iv.1987, J. Huisman, 1 ô (UMSP); Interior Zone, Tenom, $16 \mathrm{~km}$ NE Agr. Res. Station, $200 \mathrm{~m}$, 14-16.xii.1989, J. Huisman, $10^{\star}, 2$ (RMNH); Sg. Malabit, rd. Long Pa Sia to Long Semado, $04^{\circ} 21^{\prime} \mathrm{N}, 115^{\circ} 41^{\prime} \mathrm{E}$, 1175 m, 18-19.xii.1986, J. Huisman, 10 , 1 ㅇ (UMSP).

Chimarra terramater is similar to C. tityos Malicky, C. fuilianae, sp. n., and C. xiphosella, sp. n. All of these species have the character combination of having the lateral lobe of tergum $\mathrm{X}$ with a projecting and somewhat upturned ventral margin, sensilla on a rounded projection at midlength on the same structure, an inferior appendage with a projection from its mesal margin, and a relatively elongate ventral process on segment IX. Like C. tityos, C. terramater has the apex of the lateral lobes of tergum X sharply upturned and acute (typically more so than in the illustration provided by Malicky), but differs in lacking an acutely projecting basoventral projection on the inferior appendages. The overall morphology, and especially the inferior appendages are most similar to $C$. fuilianae, but in the material examined the basal part of the inferior appendage always seems to have an angular inflection. This is not apparent in the original illustration of the species, but the other characters, especially the overall shape of tergum X, seem to conform closely to those of the specimen illustrated here. Chimarra terramater also seems to differ diagnostically from $C$. fuilianae in that the ventral process of segment IX is distinctly shorter.

Adult. Color (in alcohol) yellowish-brown. Length of forewing: male $3.5-4.5 \mathrm{~mm}$, female 3.9-4.6 $\mathrm{mm}$. Forewing venation: stem of Rs curved, with enlarged, sclerotized node at inflection; fork at base of discal cell distinctly thickened, asymmetric, length of discal cell about 2 times width; $m$ crossvein proximal to crossveins $s$ and $r-m$, crossvein $s$ not hyaline; $2 \mathrm{~A}$ vein intersecting $3 \mathrm{~A}$ vein (apparently forked apically). Postocular parietal sclerite short. Maxillary palps moderately elongate, 3 distinctly longer than 2, 5 slightly shorter than 3. Protarsal claws of male not or very little enlarged, symmetrical.

Male genitalia. Abdominal segment VIII moderate in length; tergum about as long as sternum, unmodified; sternum VIII with slight posteroventral projection. Abdominal segment IX short dorsolaterally, tergum short dorsally, with pair of distinct apodemes from 

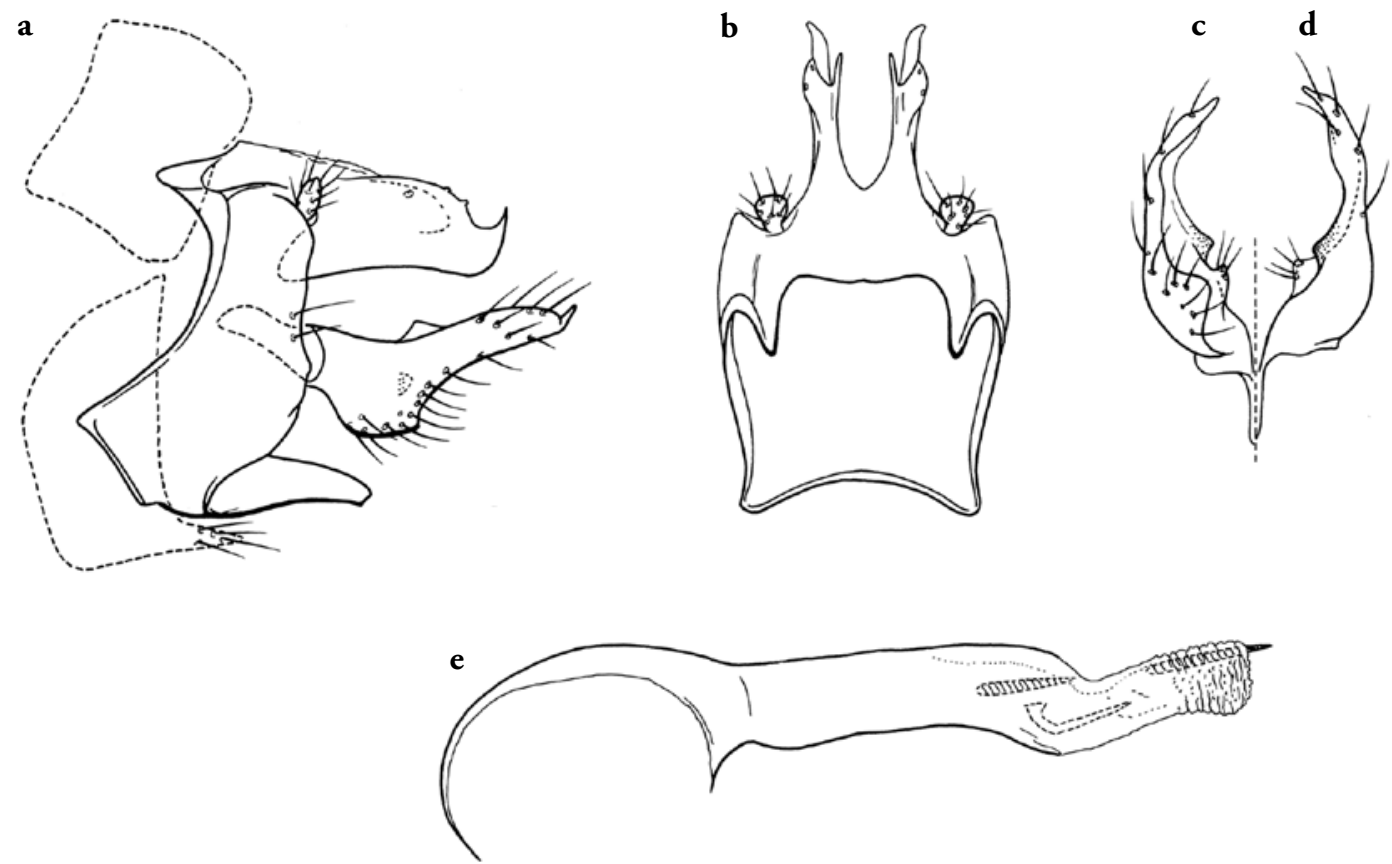

Fig. 10. Chimarra terramater, male genitalia. - a, lateral; b, segment IX and tergum $X$, dorsal; c, inferior appendage, ventral; $\mathrm{d}$, inferior appendage, dorsal; e, phallic apparatus, lateral.

anterolateral margin; anteroventral margin in lateral view distinctly produced, angular; posterior margin nearly straight; ventral process projecting posteriorly, elongate, tapering, rounded to subtruncate apically. Preanal appendage setose, short, somewhat flattened. Inferior appendage slightly longer than tergum X; in lateral view strongly dorsally inflected basoventrally, widest basally, narrowing distally, basoventral margin rounded, apex acute; in ventral view mesally curved, basomesally with slightly angulate projection. Tergum $\mathrm{X}$ with sclerotized lateral lobes and projecting mesal membranous lobe; lateral lobe moderately long, with projecting ventral margin, lobe bearing 2 seta-like sensilla, sensilla on slightly projecting, short, rounded middorsal lobe, apex of ventral margin distinctly hooked dorsally. Phallobase tubular, with pronounced basodorsal expansion, moderately elongate, ventral margin slightly bulged basally, ventral apex weakly sclerotized. Endotheca elongate, tubular, with granularly textured region and 2 short, subequal endothecal spines. Phallotremal sclerite complex composed of rod and ring structure, rod moderately elongate.

\section{Chimarra tityos Malicky}

Fig. 11

Chimarra tityos Malicky, 2008: 839, holotype 0 , Indonesia (Eastern Kalimantan), Zoologische Museum Lausanne.

Material examined. Malaysia, Sarawak, Gunung Gading National Park, Sg. Lundu, 23.-26.x.2003, W. Mey,

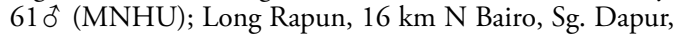
$03^{\circ} 53^{\prime} \mathrm{N}, 115^{\circ} 35^{\prime} \mathrm{E}, 1200 \mathrm{~m}, 19-22 . i i .1987$, J. Huisman, $10^{\widehat{T}}$ (UMSP). Sabah, $60 \mathrm{~km}$ W Lahad Datu, Danum Valley Field Centre, Sg. Palum Tambun \& vicinity, $04^{\circ} 58^{\prime} \mathrm{N}$, $117^{\circ} 48^{\prime} \mathrm{E}, 150$, 9.xi.1986, J. Huisman $10^{\circ}$ (RMNH); Crocker Range National Park, Sg. Myoog, 8.xi.2003, W. Mey, 90ิ, 6 ㅇ (MNHU); Ranau, 20 km E Sg. Bayaan, 6.xi.2003, W. Mey, 40, 1 ㅇ (MNHU); Danum Valley Field Centre, Sg. Palum Tambun, 18-19.viii.2005,

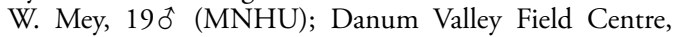
Sg. Kalisun, 17.viii.2005, W. Mey, 140, 1 우 (MNHU); same locality, 2.xi.2003, W. Mey, 10; Danum Valley Field Centre, Tembaling trail, 18.viii.2005, W. Mey, $1 \delta^{\hat{\sigma}}$ (MNHU); Tawau Hills National Park, Sg. Tawau, 1921.viii.2005, W. Mey, 80 , 2 ( Sg. Matang, $04^{\circ} 24^{\prime} \mathrm{N}, 115^{\circ} 43^{\prime} \mathrm{E}$, 6.xii.1987, Huisman \& Achterberg, 10 , 1 ㅇ (RMNH); SE Sabah nr. Danum Valley Field Center, 240 m, 18.x-22.xi.1987, Achterberg, 10 , 1 i (RMNH); $60 \mathrm{~km}$ W Lahad Datu, Danum Valley Field Centre, Sg. Palum Tambun \& vicinity, $04^{\circ} 58^{\prime} \mathrm{N}, 117^{\circ} 48^{\prime} \mathrm{E}$, 150, 9.xi.1986, J. Huisman, 20 (RMNH); same locality, 

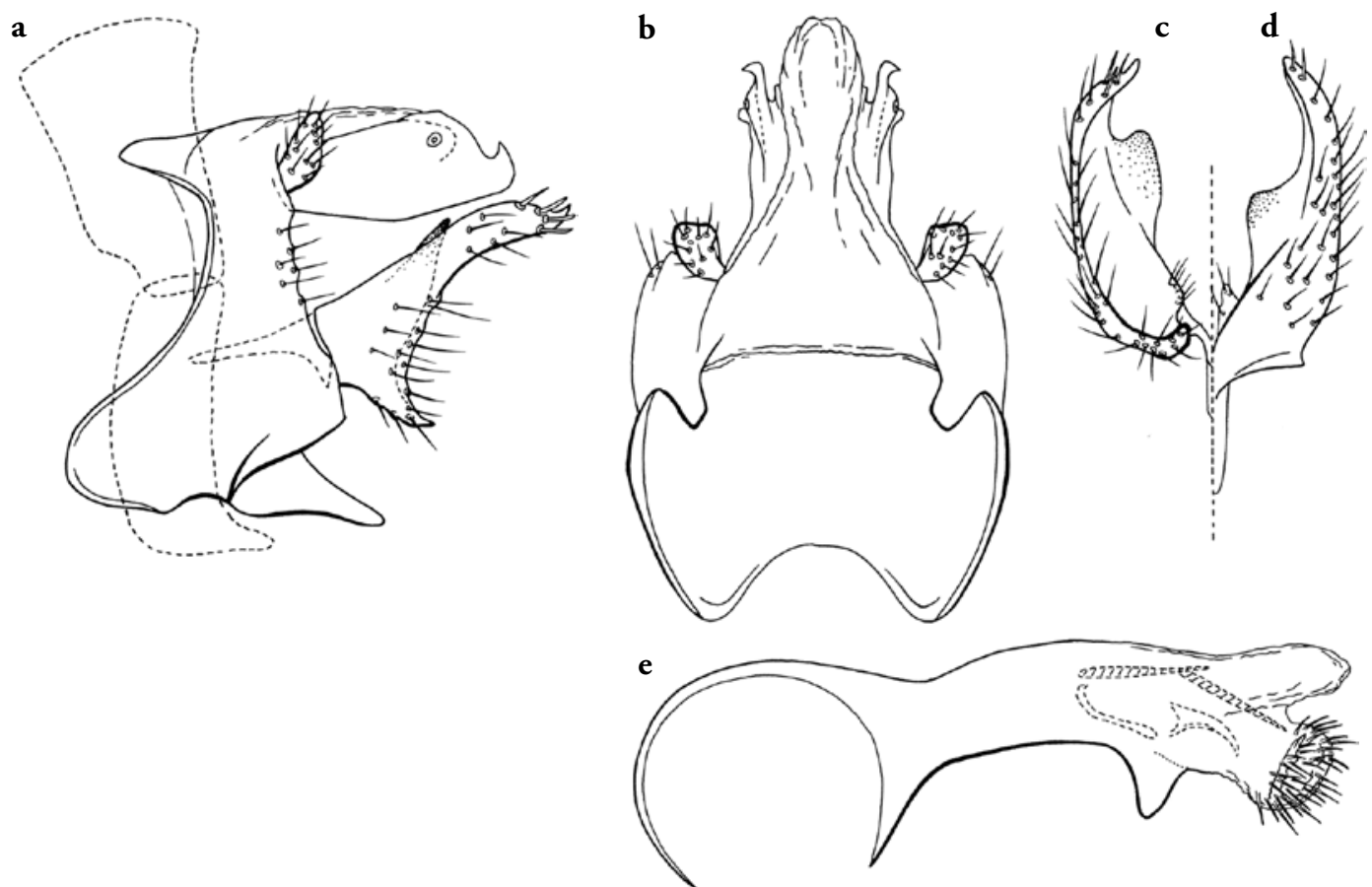

Fig. 11. Chimarra tityos, male genitalia. - $a$, lateral; $b$, segment IX and tergum $X$, dorsal; c, inferior appendage, ventral; $d$, inferior appendage, dorsal; e, phallic apparatus, lateral.

19-26.iii.1987, J. Huisman, 3ㅊ, 19 (UMSP); Long Pa Sia airstrip, $04^{\circ} 24^{\prime} \mathrm{N}, 115^{\circ} 43^{\prime} \mathrm{E}, 1000 \mathrm{~m}, 15 . \mathrm{iv} .1987$, Huisman \& van Tol, 10 (UMSP); $60 \mathrm{~km} \mathrm{~W} \mathrm{Lahad}$ Datu, Danum Valley Field Centre, nature trail brooklet, $04^{\circ} 58^{\prime} \mathrm{N}, 117^{\circ} 48^{\prime} \mathrm{E}, 180 \mathrm{~m}$, 9-13.ix.1986, J. Huisman, $5 \sigma^{\widehat{T}}$ (UMSP); Poring Hot Spring, $11 \mathrm{~km}$ NNE Ranau, Sg. Montokungon crossing road, 06 $02^{\prime} 30^{\prime \prime} \mathrm{N}, 116^{\circ} 43^{\prime} \mathrm{E}$, 450 m, 25.xi.1986, J. Huisman, $180^{\circ}$ (UMSP); same locality, 28.i.1987, J. Huisman, 12 $\widehat{0}, 23$ ㅇ (UMSP); Poring Hot Spring, $12 \mathrm{~km}$ NNE Ranau, confluence Sg. Kepungit $\&$ Sg. Langanan, $06^{\circ} 03^{\prime} \mathrm{N}, 116^{\circ} 43^{\prime} \mathrm{E}, 450 \mathrm{~m}$, 8.xii.1986, J. Huisman, $10^{\text {to }} 1$ 우 (RMNH); $20 \mathrm{~km}$ NNE Ranau, Kg. Takutan, Sg. Mokodou, 06 $06^{\prime} \mathrm{N}, 116^{\circ} 45^{\prime} \mathrm{E}, 325 \mathrm{~m}$, 4.ii.1987, J. Huisman, 20, 2 우 (UMSP); $10 \mathrm{~km} \mathrm{SE}$ Ranau, Kg. Nalapak, Sg. Kananapun, 0559'N, 116 $47^{\circ} \mathrm{E}$, 350 m, 7.ii.1987, J. Huisman, 7 ㅈ, 4 우 (UMSP); Long Pa Sia, airstrip, $04^{\circ} 24^{\prime} \mathrm{N}, 115^{\circ} 43^{\prime} \mathrm{E}, 1000 \mathrm{~m}$, 16.iv.1987, Huisman \& van Tol, $1 \sigma^{\star}(\mathrm{RMNH}) ; 2 \mathrm{~km}$ SW Long Pa Sia, Long Rurun (Sg. Ritan), 04²3'N, 11542'E, 1040 m, 5.xii.1987, Huisman \& Achterberg, 20 , 1 우 (RMNH); $12 \mathrm{~km}$ NNE Ranau, Poring Hot Spring, staff quarters, $06^{\circ} 03^{\prime} \mathrm{N}, 116^{\circ} 42^{\prime} \mathrm{E}, 550 \mathrm{~m}, 1 . i \mathrm{i} .1987$, Huisman \& de Jong, 1 đิ (RMNH); Sg. Moyig at tributary, $8 \mathrm{mi}$ E Penampung, 1.x.1978 G. \& C. Edmunds, 17 ऊ (NMNH); Tenom, 1.ix.1983, Steiner \& Hevel, 30 (NMNH); Ranau, 13.viii.1983, Steiner \& Hevel, 30 , 7 우 (NMNH).

Chimarra tityos is most similar to C. fuilianae, sp. n. and $C$. terramater Malicky. It resembles these species in having an angulate, sclerotized mesal projection from the inferior appendage and in having the lateral lobes of tergum X with upturned apices. Distinctive differences include a preapical ventral projection from the phallobase, a more prominent apicoventral projection from the inferior appendage, a less elongate ventral process on segment IX, and a distinctive spinose tract on the endotheca.

Adult. Color (in alcohol) light brown. Length of forewing: male 4.0-4.8 mm, female 4.0-5.2 $\mathrm{mm}$. Forewing venation: stem of Rs curved, with enlarged, sclerotized node at inflection; fork at base of discal cell distinctly thickened, asymmetric, length of discal cell about 2.5 times width; $m$ crossvein proximal to crossveins $s$ and $r-m$, crossvein $s$ not hyaline; 2A vein intersecting 3A vein (apparently forked apically). Postocular parietal sclerite short. Maxillary palps moderately elongate, segment 3 distinctly longer than 2, 3 subequal to 5. Protarsal claws of male not or very little enlarged, symmetrical.

Male genitalia. Abdominal segment VIII short; tergum about as long as sternum, unmodified, entire, without mesal excavation; sternum VIII with slight posteroventral projection. Abdominal segment IX short dorsolaterally, tergum short dorsally, with pair of distinct apodemes from anterolateral 

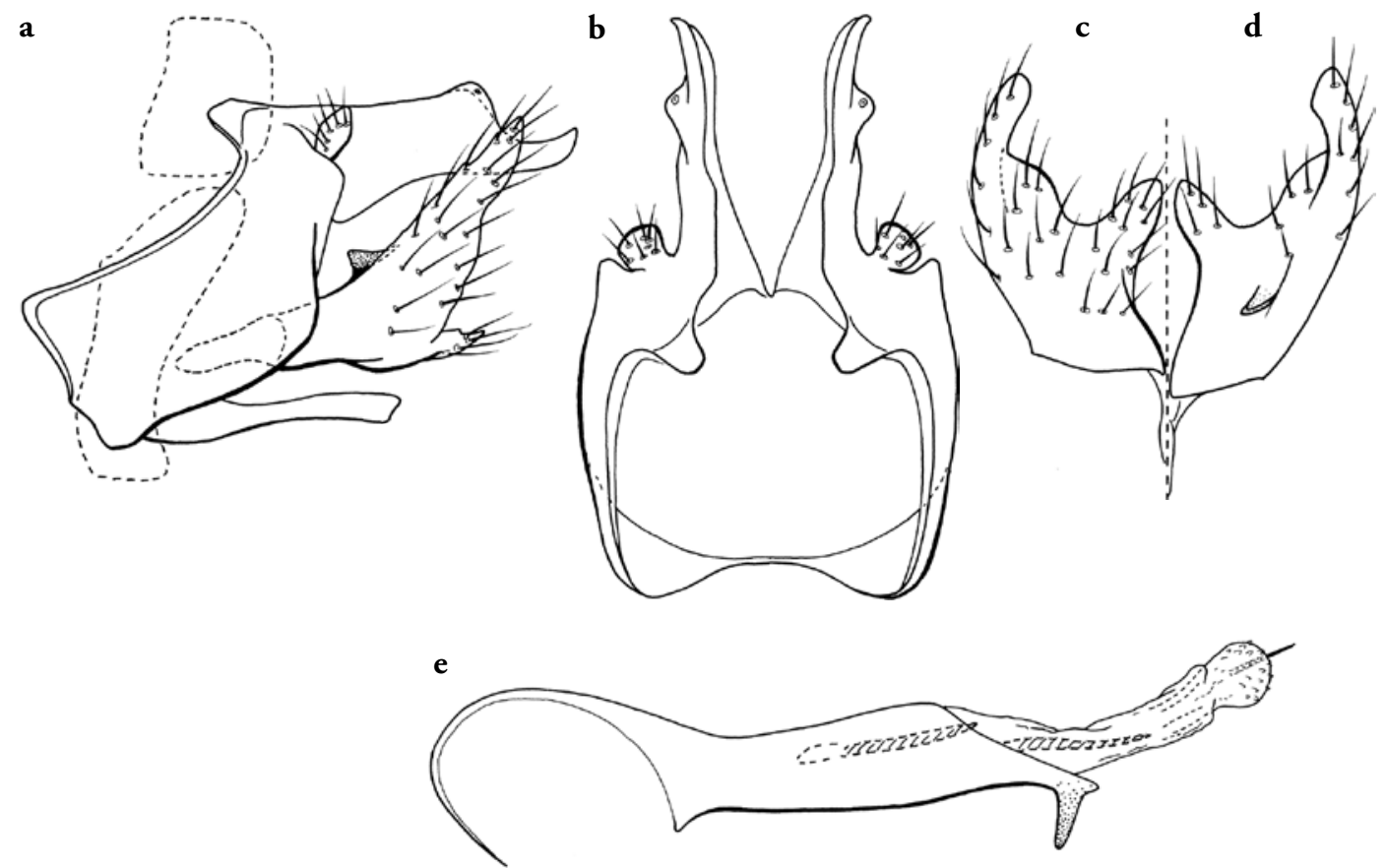

Fig. 12. Chimarra xiphosella, male genitalia. - a, lateral; b, segment IX and tergum $X$, dorsal; c, inferior appendage, ventral; $\mathrm{d}$, inferior appendage, dorsal; e, phallic apparatus, lateral.

margin; anteroventral margin in lateral view produced, rounded; posterior margin nearly straight; ventral process projecting posteriorly, elongate, narrowing from base, acute apically. Preanal appendage setose, short, rounded or subquadrate, somewhat flattened, slightly narrowed basally. Inferior appendage about as long as tergum $\mathrm{X}$; in lateral view dorsally inflected basoventrally, widest basally, narrowing distally, apex subacute; in ventral view mesally curved, basally with acute projection from posterior margin, mesal surface with broad tooth-like projection at midlength. Tergum X with sclerotized lateral lobes and projecting mesal membranous lobe; lateral lobe moderately long, with projecting ventral margin, lobe bearing 2 sensilla, sensilla on slightly projecting, short, rounded middorsal lobe, apex of ventral margin distinctly hooked dorsally. Phallobase tubular, with pronounced basodorsal expansion, relatively short, ventral apex weakly sclerotized, with strongly deflexed ventral process. Endotheca length not discernable (not expanded), with tract of numerous needle-like spines near apex; and 2 fine, short, subequal endothecal spines. Phallotremal sclerite complex composed of rod and ring structure, rod very short, apically with pair of distinct, curved, lateral sclerites.

\section{Chimarra xiphosella sp. $\mathbf{n}$.}

Fig. 12

Type material. Holotype: $\widehat{\star}$, Malaysia, Sabah, $9.5 \mathrm{~km}$ NNW Kundassang, Marei-Parei, Sg. Tawubang, $06^{\circ} 04^{\prime} \mathrm{N}, 116^{\circ} 30^{\prime} \mathrm{E}, 1030 \mathrm{~m}, 11$. iii.1987, J. Huisman (UMSP000107141) (RMNH).

Chimarra xiphosella is similar to C. fuilianae, sp. n., C. terramater Malicky, and C. tityos Malicky. All of these species have the character combination of having the lateral lobe of tergum $\mathrm{X}$ with a projecting and somewhat upturned ventral margin, sensilla on a rounded projection at midlength on the same structure, an inferior appendage with a projection from its mesal margin, and a relatively elongate ventral process on segment IX. Among these, C. xiphosella is particularly distinctive, and easily diagnosed by the shape of the inferior appendages, each of which has a prominent apicoventral projection, and by the very elongate ventral process of segment IX.

Adult. Characters unknown, only abdomen existing. Male genitalia. Abdominal segment VIII short; tergum about as long as sternum, unmodified; sternum VIII without posteroventral projection. 
Abdominal segment IX relatively wide dorsolaterally, tergum short dorsally, with pair of distinct apodemes from anterolateral margin; anteroventral margin in lateral view distinctly produced, angular; posterior margin slightly produced; ventral process projecting posteriorly, very elongate, nearly uniform in width, subtruncate apically. Preanal appendage setose, short, rounded or subquadrate, somewhat flattened, slightly narrowed basally. Inferior appendage about as long as tergum $\mathrm{X}$; in lateral view dorsally inflected basoventrally, widest basally, narrow distally, basoventral margin with acute projection, apex rounded; in ventral view somewhat mesally curved, basally with strong, thumb-like projection from posterior margin, mesal surface with tooth-like projection at midlength. Tergum $\mathrm{X}$ with sclerotized lateral lobes only, mesal membrane not evident, apparently lacking; lateral lobe long, with projecting ventral margin, lobe bearing 2 sensilla, sensilla on slightly projecting, rounded middorsal lobe, apex of ventral margin narrow, subacute. Phallobase tubular, with pronounced basodorsal expansion, moderately elongate, ventral apex distinctly sclerotized, distinctly projecting, with strongly deflexed, acute ventral process. Endotheca elongate, tubular, with granularly textured region and 3 endothecal spines, 2 subequal, moderately long basal spines and 1 very short, slender apical spine. Phallotremal sclerite complex not evident in specimen examined.

Etymology. This species is named Chimarra xiphosella, as a diminutive derived from the Greek word xiphos, a sword, and referring to the elongate ventral process of segment IX; to be treated as a noun in apposition.

\section{Chimarra tsudai group}

The Chimarra tsudai group is more geographically restricted than the Chimarra digitata group, ranging from Pakistan to Japan and into Southeast Asia and parts of Indonesia. About 130 species, or over half the total Chimarra species from the Oriental region, can be confidently placed here. These are listed in Table 1 . The most diagnostic attribute is that the lateral lobes of tergum X of the male genitalia are subdivided into sclerotized lateral and mesal lobes, with the mesal pair membranous basally and forming upright, digitate processes that straddle the dorsally directed phallic apparatus (Fig. 15A, B). As noted by Ross (1956), the group is also characterized by having the lobes of tergum $\mathrm{X}$ with numerous sensilla (many more than 2). These are always located on the lateral lobes (Fig. $14 \mathrm{~A}, \mathrm{~B}$ ) and often on the sclerotized mesal lobes as well (Fig. 24A, B). Another diagnostic character of this group is that the dorsomesal part of segment IX is obsolete or membranous, and there is no membranous mesal lobe of tergum X (Fig. 22B). Additionally, the ventral process of segment IX is either short and deflected ventrally (rather than posteriorly) (Fig. 15A) or absent (Fig. 14A). A subgroup of the Chimarra tsudai Group, including many species from Borneo, has tergum VIII highly modified and apically margined with spines or spine-like projections, thus superficially resembling species in the subgenus Curgia (Fig. 16A, G). The forewing venation typically has the stem of Rs deflected (curved downward) and the fork at the base of the discal cell more or less symmetrical (Figs 41-43). The forewing discal cell is elongate (length often 3 times the width or more) and both veins $2 \mathrm{~A}$ and $3 \mathrm{~A}$ are looped to $1 \mathrm{~A}$ (Figs 41-43). The latter character also occurs in the subgenera Curgia, Otarrha, and some Chimarrita, as well as in species of the Chimarrhafra lineage of Africa, but not typically in the Chimarra digitata group, in which $2 \mathrm{~A}$ appears to be forked or Y-shaped apically (Figs 39A, 40A).

Adult. Forewing Rs deflected or curved (rather than sinuous or S-shaped); basal fork of discal cell more or less symmetrical; discal cell elongate, length often 3 or more times width; $m$ crossvein usually proximal to $s$ and $r-m$ crossveins, sometimes nearly linearly arranged; $s$ hyaline or not; $2 \mathrm{~A}$ obsolete apically, apparently looped to $1 \mathrm{~A}$. $\delta$ with foretarsi and claws (probably) always enlarged.

Male genitalia (Fig. 15). Segment VIII unmodified or modified; if modified, then often enlarged, with posteromesal margin invaginated and margined apically with projecting spine-like or scabrous processes. Segment IX with anteromesal margin slightly to distinctly projecting, usually rounded mesally (rather than sinuate); anterodorsal apodemes weakly developed or absent; dorsomesal margin obsolete, membranous; ventral process broad basally, short, ventrally deflected, absent in some species. Tergum $\mathrm{X}$ without membranous mesal lobe; lateral lobes divided into sclerotized lateral and mesal lobes, lateral lobes variable in shape, always with numerous sensilla, mesal lobes membranous basally, forming upturned digitate processes, sometimes with apical sensilla. Preanal appendages short, setose, often very small. Inferior appendages variable in length and shape, usually relatively linear as viewed laterally, with little evidence of basal inflection; linear or mesally curved, as viewed ventrally, typically with acute apical or preapical tooth (or teeth) or acute dorsal or dorsomesal projection. Phallic apparatus with phallobase tubular, long or short, ventral apex projecting or not; endotheca variable in length, short or 
Table 1. Chimarra tsudai group species.Placement in tsudai group not ascertainable from original description: Chimarra auronitens Ulmer, 1906; Chimarra pedalis Banks, 1931; Chimarra pilosella Navás, 1932; Chimarra tagalica Banks, 1937.

Chimarra aberrans Martynov, 1935

Chimarra alcicorne Malicky, 1995

Chimarra aminadab Malicky, 1993

Chimarra ammi Malicky, 2008

Chimarra anam Malicky, 2008

Chimarra aneca Malicky \& Chantaramongkol, 1993

Chimarra areli Malicky, 2008

Chimarra argeia Malicky \& Chantaramongkol, 1997

Chimarra arkit Malicky, 2008

Chimarra atara Malicky \& Chantaramongkol, 1993

Chimarra atnia Malicky \& Chantaramongkol, 1993

Chimarra atripennis Banks, 1931

Chimarra auricoma Kimmins, 1957

Chimarra batukaua Malicky, 1995

Chimarra berenike Malicky, 1998

Chimarra biatec Malicky, 1993

Chimarra burmana Kimmins, 1957

Chimarra caduca Blahnik et al., sp. n.

Chimarra chanchuluni Blahnik et al., sp. n.

Chimarra concava Kimmins, 1957

Chimarra concolor Ulmer, 1905

Chimarra confusa Ulmer, 1907

Chimarra crepidata Kimmins, 1957

Chimarra cumata Malicky \& Chantaramongkol, 1993

Chimarra cuspidata Blahnik et al., sp. n.

Chimarra cygnus Blahnik et al., sp. n.

Chimarra danumensis Blahnik et al., sp. n.

Chimarra denticula Blahnik et al., sp. n.

Chimarra devva Malicky \& Chantaramongkol, 1993

Chimarra dirke Malicky \& Thamsenanupap, 2000

Chimarra discolor Kimmins, 1957

Chimarra excavata Kimmins, 1957

Chimarra fansipangensis Mey, 1998

Chimarra fenestrata Kimmins, 1964

Chimarra flaviventris Kimmins, 1957

Chimarra fluctuata Sun, 2007

Chimarra fulmeki Ulmer, 1951

Chimarra fusca Kimmins, 1957

Chimarra gemmal Malicky, 1989

Chimarra gigama Malicky, 1989

Chimarra gunungkawi Malicky, 1995

Chimarra haimuoi Malicky, 1995

Chimarra haimuoiba Malicky, 1995

Chimarra haimuoibon Malicky, 1995

Chimarra haimuoimot Malicky, 1995

Chimarra haimuoinam Malicky, 1995

Chimarra hezron Malicky, 1993

Chimarra hoangliensis Mey, 2005

Chimarra horok Malicky, 1989

Chimarra houvichka Schmid, 1960

Chimarra htinorum Chantaramongkol \& Malicky, 1989

Chimarra igvarvaria Melnitsky, 2005

Chimarra inthanonensis Chantaramongkol \& Malicky,

1989

Chimarra jacobsoni Ulmer, 1951
Chimarra jannekae Blahnik et al., sp. n.

Chimarra jaroschi Malicky, 1994

Chimarra jisipu Malicky, 1989

Chimarra joliveti Jacquemart, 1979

Chimarra kailishchandrai Malicky, 1997

Chimarra karlijnae Blahnik et al., sp. n.

Chimarra khasia Kimmins, 1957

Chimarra kinabaluensis Blahnik et al., sp. n.

Chimarra kumaonensis Martynov, 1935

Chimarra lahuorum Chantaramongkol \& Malicky, 1989

Chimarra lambi Blahnik et al., sp. n.

Chimarra lannaensis Chantaramongkol \& Malicky, 1989

Chimarra lichiuensis Hsu \& Chen, 1996

Chimarra litugena Malicky \& Chantaramongkol, 1993

Chimarra litussa Malicky \& Chantaramongkol, 1993

Chimarra liwaguensis Blahnik et al., sp. $\mathrm{n}$.

Chimarra longispina Sun, 2007

Chimarra maoi Sun \& Malicky, 2002

Chimarra malaisei Kimmins, 1957

Chimarra mars Malicky, 2007

Chimarra matura Malicky \& Chantaramongkol, 1993

Chimarra megara Malicky, 2007

Chimarra meorum Chantaramongkol \& Malicky, 1989

Chimarra mlabriorum Chantaramongkol \& Malicky, 1989

Chimarra momma Malicky \& Chantaramongkol, 1993

Chimarra mommaides Mey, 1998

Chimarra nahesson Malicky \& Chantaramongkol, 1993

Chimarra nepalensis Kimmins, 1964

Chimarra nigra Kimmins, 1964

Chimarra nigrorosea Schmid, 1960

Chimarra noloyan Blahnik et al., sp. n.

Chimarra nonna Malicky, 1993

Chimarra nunenada Melnitsky, 2005

Chimarra opaca Mey, 1998

Chimarra oreithyia Malicky, 2007

Chimarra palawana Malicky, 1994

Chimarra pasiphae Malicky, 2007

Chimarra pataplan Malicky, 1989

Chimarra phillipsae Blahnik et al., sp. $\mathrm{n}$.

Chimarra physanoton Blahnik et al., sp. n.

Chimarra podarge Malicky \& Thamsenanupap, 2007

Chimarra pontos Malicky, 2007

Chimarra preapicalis Blahnik et al., sp. n.

Chimarra prokrustes Malicky, 2008

Chimarra rama Malicky \& Chantaramongkol, 1993

Chimarra ravanna Malicky \& Chantaramongkol, 1993

Chimarra schwendingeri Chantaramongkol \& Malicky,

1989

Chimarra scolops Blahnik et al., sp. n.

Chimarra scopulifera Kimmins, 1957

Chimarra semiramis Malicky, 2007

Chimarra senticosa Sun \& Malicky, 2002

Chimarra shiva Malicky \& Chantaramongkol, 1993

Chimarra silausilau Blahnik et al., sp. $\mathrm{n}$.

Chimarra sinitorum Blahnik et al., sp. n. 
Chimarra sita Malicky \& Chantaramongkol, 1993

Chimarra spinifera Kimmins, 1957

Chimarra spitzeri Malicky, 1994

Chimarra stenodactylus Blahnik et al., sp. n.

Chimarra suadulla Malicky \& Chantaramongkol, 1993

Chimarra supanna Malicky, 1993

Chimarra suryasena Schmid, 1960

Chimarra suthepensis Chantaramongkol \& Malicky, 1989

Chimarra sythoffi Ulmer, 1951

Chimarra talos Malicky, 2007
Chimarra thaumas Malicky, 2008

Chimarra triangulata Hsu \& Chen, 1996

Chimarra tsudai Ross, 1956

Chimarra uncula Mey, 1998

Chimarra uschtu Malicky, 1989

Chimarra vantoli Blahnik et al., sp. n.

Chimarra vanwelzeni Blahnik et al., sp. $\mathrm{n}$.

Chimarra vesta Malicky, 2007

Chimarra wushikangensis Hsu \& Chen, 1996

Chimarra yaorum Chantaramongkol \& Malicky, 1989 elongate,sometimestexturedwithsmallpapillaeorwith tracts of numerous short spines, variably positioned in different species; phallotremal sclerite complex variable, either small, forming typical short rod and ring structure, or enlarged and sclerotized, sometimes with accompanying dorsolateral or apical sclerites.

\section{Chimarra atripennis Banks}

Chimarrha atripennis Banks, 1931: 425, holotype +, Malaysia (Sabah), MCZT.

This species was originally described from Mt. Kinabalu, based on specimens from Marei Parei, $5000 \mathrm{ft}$. and Kiau, $3000 \mathrm{ft}$. Two specimens were indicated in the type series, but their sex was not stated. Also, no illustration was provided. A specimen labeled as the holotype (MCZT 16506) was borrowed from the Museum of Comparative Zoology, Harvard University, and examined by W. Mey (pers. com.). It is a female. The species is large (wing expanse $13 \mathrm{~mm}$ ) and black in color. The description of the venation, Rs curved downward, discal cell elongate, makes it evident that this is a species in the Chimarra tsudai group. It is likely that it is one of the species described in this paper, but until females are definitively associated and described, its identity will remain in doubt.

\section{Chimarra caduca sp. $\mathbf{n}$.}

Fig. 13

Type material. Holotype: $\delta$, Malaysia, Sabah, Crocker Range, $40 \mathrm{~km} \mathrm{~S}$ of Kota Kinabalu, Sinsuron Rd., 1500 m, 19.xii.1989, J. Huisman (UMSP000107300) (RMNH).

Paratypes. Malaysia, Sabah, Kinabalu National Park, headquarters, $1500 \mathrm{~m}, 10$. viii.2005, W. Mey, 40 (UMHU).

Chimarra caduca also belongs to the subgroup of the Chimarra tsudai group in which tergum VIII is highly modified, invaginated mesally and variously armed with spine-like projections. Generalized features it possesses are a phallotremal sclerite that is not greatly enlarged, and an endotheca that is relatively short and lacks distinctive spines. Species in this group are all distinguished by characteristic differences in the shapes of the lateral and mesal lobes of tergum $\mathrm{X}$ and in the location of the mesal projection of the inferior appendages. In C. caduca, the mesal projection of the inferior appendages is located just past midlength. In this respect, it is similar to $C$. jannekae, sp. n. and C. cygnus, sp. n., both of which differ significantly in the shapes the lobes of tergum $\mathrm{X}$, as well as in details of the phallic apparatus.

Adult. Color (in alcohol) light brown. Length of forewing: male $5.5 \mathrm{~mm}$. Forewing venation: stem of Rs only slightly curved; fork at base of discal cell not thickened, nearly symmetrical, length of discal cell about 3 times width; $m$ crossvein somewhat proximal to crossveins $s$ and $r$ - $m$, crossvein $s$ not hyaline; $2 \mathrm{~A}$ vein not intersecting $3 \mathrm{~A}$ (apparently looped to 1A). Postocular parietal sclerite moderately elongate. Maxillary palps moderately elongate, segment 3 subequal to 2 and 5. Protarsal claws of male enlarged, asymmetrical in shape and length.

Male genitalia. Abdominal segment VIII short; tergum about as long as sternum, modified, with small mesal excavation, excavation bordered laterally with laciniate fringe, lateral fringe more elongate; sternum VIII without posteroventral projection. Abdominal segment IX short dorsolaterally; anteroventral margin in lateral view slightly produced, rounded; posterior margin sinuously produced; ventral process projecting ventrally, short, broad basally, broadly rounded. Preanal appendage setose, short, rounded, broadest basally. Inferior appendage about as long as tergum X; in lateral view straight, nearly uniformly wide, apex rounded; in ventral view nearly straight, dorsomesally with broad, distinctly sclerotized, apically acute projection just beyond midlength. Tergum $\mathrm{X}$ with sclerotized lateral lobes and separate mesal lobes, mesal membrane lacking; lateral lobe long, in lateral view narrow, apex cupped, slightly 

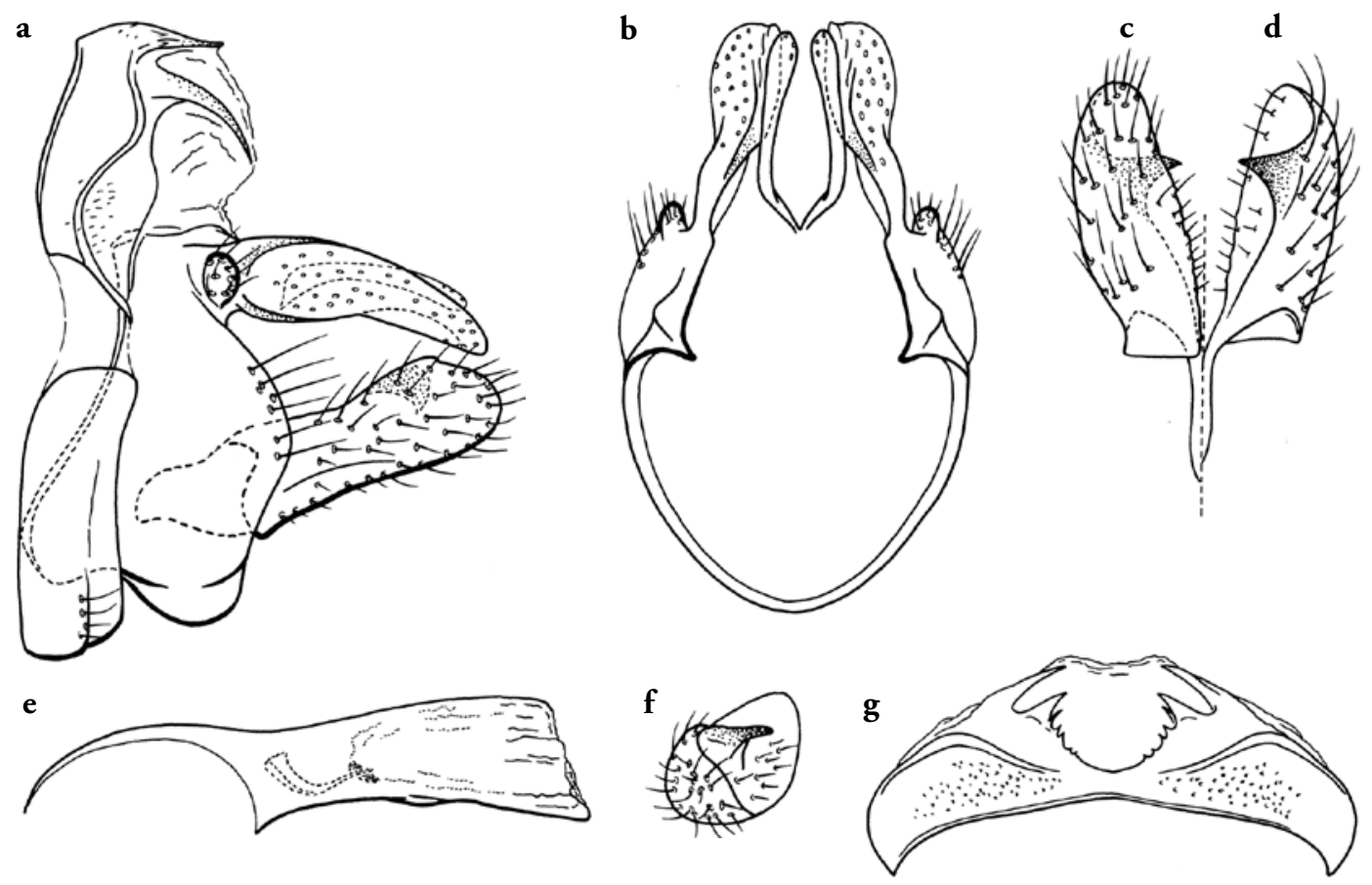

Fig. 13. Chimarra caduca, male genitalia. - a, lateral; b, segment IX and tergum X, dorsal; c, inferior appendage, ventral; $d$, inferior appendage, dorsal; e, phallic apparatus, lateral; $f$, inferior appendage, caudal; $f$, tergum VIII, dorsal.

downcurved, lobe bearing multiple sensilla; mesal lobe elongate, digitate, reclinate, posteriorly directed. Phallobase tubular, with pronounced basodorsal expansion, very short. Endotheca relatively short, granularly textured region not discernable, without endothecal spines. Phallotremal sclerite composed of rod and ring structure, rod very short, apically with pair of short, curved, lateral sclerites.

Etymology. This species is named Chimarra caduca from the Latin word caducus, meaning "that falls or is inclined to fall", in reference to the relatively prostrate mesal lobes of tergum $\mathrm{X}$ of this species.

\section{Chimarra chanchuluni sp. $\mathrm{n}$.}

Fig. 14

Type material. Holotype: $\hat{\sigma}$, Malaysia, Sabah, Kinabalu National Park, Headquarters, Sg. Liwagu @ Silau-Silau trail, $06^{\circ} 00^{\prime} \mathrm{N}, 116^{\circ} 33^{\prime} \mathrm{E}, 1470 \mathrm{~m}$, 22.i.1987, Huisman \& de Jong (UMSP000107181) (RMNH).

Paratypes. Malaysia, Sabah, same data as holotype, 10 , 4 ㅇ (RMNH); Crocker Range, 5 km N Tenom, base of Sg. ulu Noloyan, $05^{\circ} 10^{\prime} \mathrm{N}, 115^{\circ} 56^{\prime} \mathrm{E}, 1010 \mathrm{~m}$, 10-11.x.1986, J. Huisman, $60^{\star}$ (UMSP).
Chimarra chanchuluni is a distinctive species with a number of diagnostic characters, including the shape of the inferior appendages, which are somewhat dorsoventrally flattened, mesally curved, as viewed ventrally, and have a small spine-like, preapical projection on the mesal surface. Other diagnostic characters include the shape of the mesal lobes of tergum X (each of which is divided into a pair of sclerotized, spine-like processes), the elongate preanal appendages, and the form of the phallic apparatus, which is laterally compressed apically, and lacks endothecal spines.

Adult. Color (in alcohol) yellowish-brown. Length of forewing: male 5.2-6.8 mm, female $5.8-7.2 \mathrm{~mm}$. Forewing venation: stem of Rs curved, slightly sclerotized and thickened at inflection; fork at base of discal cell not thickened, nearly symmetrical, length of discal cell about 4 times width; $m$ crossvein proximal to crossveins $s$ and $r-m$, crossvein $s$ hyaline; $2 \mathrm{~A}$ vein not intersecting 3A (apparently looped to $1 \mathrm{~A}$ ). Postocular parietal sclerite moderately elongate. Maxillary palps elongate, segment 3 slightly greater than 2, 5 shorter than 3. Protarsal claws of male enlarged, asymmetrical in shape and length.

Male genitalia. Abdominal segment VIII short; tergum longer than sternum, unmodified, with 

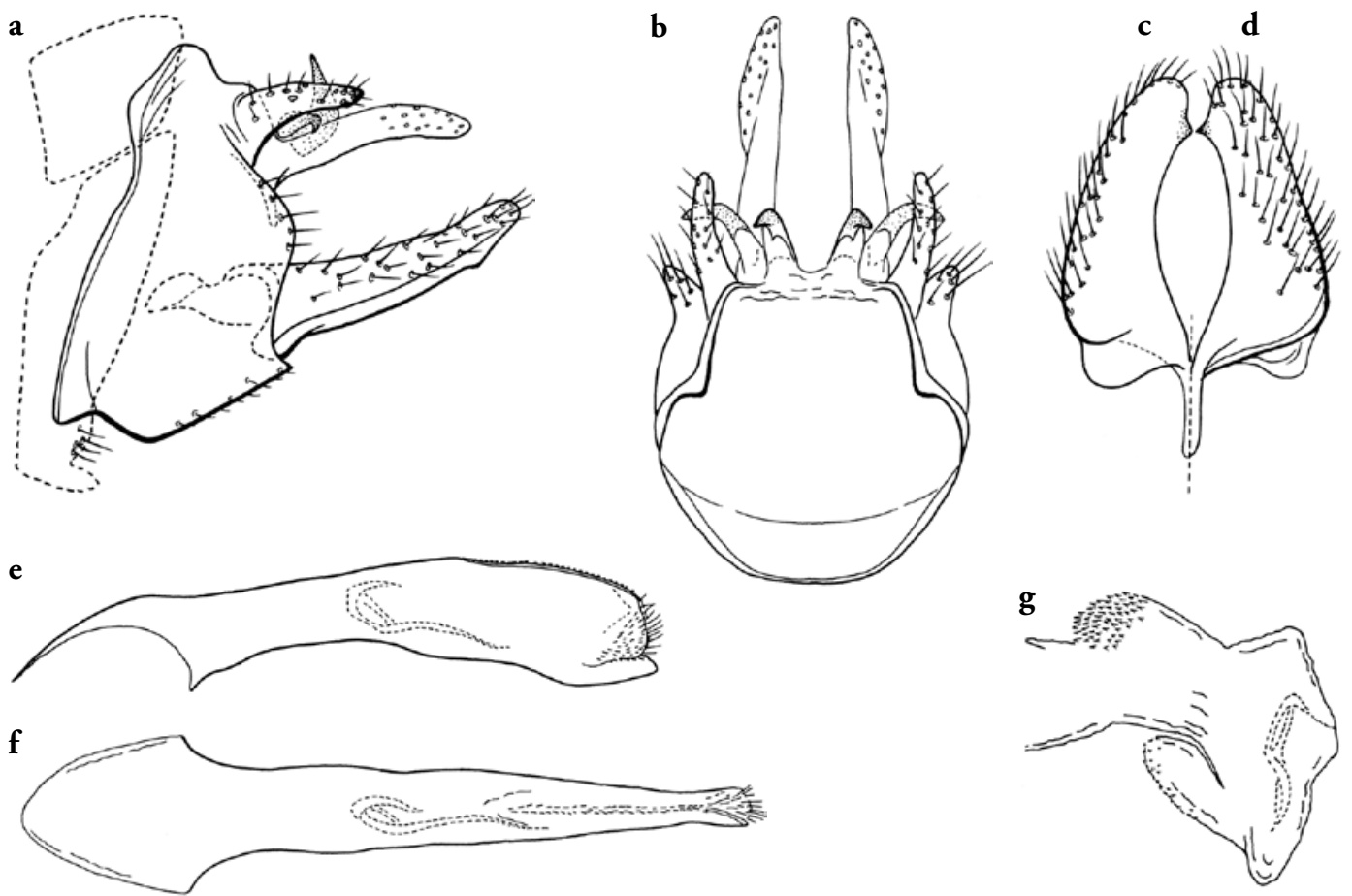

Fig. 14. Chimarra chanchuluni, male genitalia. - a, lateral; b, segment IX and tergum X, dorsal; c, inferior appendage, ventral; $\mathrm{d}$, inferior appendage, dorsal; e, phallic apparatus, lateral; $\mathrm{f}$, phallic apparatus, dorsal; g, apex of expanded endotheca, lateral.

V-shaped mesal depression; sternum VIII with slight posteroventral projection. Abdominal segment IX relatively wide dorsolaterally, tergum obsolete dorsomesally, without distinct anterolateral apodemes; anteroventral margin in lateral view not, or scarcely produced; posterior margin sinuously produced; ventral process absent or nearly so. Preanal appendage setose, moderately elongate, broadest basally, tapering and rounded apically. Inferior appendage slightly longer than tergum $\mathrm{X}$; in lateral view straight, nearly uniformly wide, apex rounded; in ventral view somewhat mesally curved, preapically with small, acute, mesal projection. Tergum $\mathrm{X}$ with sclerotized lateral and mesal lobes, mesal membrane lacking; lateral lobe moderately long, in lateral view narrow, apex slightly downcurved, lobe bearing multiple sensilla; mesal lobe short, distinctly sclerotized, bifid, both branches strongly hooked, mesal branch dorsally directed, lateral branch laterally directed. Phallobase tubular, with pronounced basodorsal expansion, moderately elongate, relatively narrow, laterally compressed apically, dorsal margin extended apically, elongate, narrow, lightly sclerotized. Endotheca relatively short, dorsally with bulging tract of minute spines, apicoventrally with lobe bearing minute spinules; other endothecal spines absent. Phallotremal sclerite complex composed of rod and ring structure, rod moderately elongate.

Etymology. Named for Chan Chu Lun, friend of J. Huisman.

\section{Chimarra cuspidata sp. n.}

Fig. 15

Type material. Holotype: $\widehat{o}$, Malaysia, Sabah, $60 \mathrm{~km} \mathrm{~W}$ Lahad Datu, Danum Valley Field Centre, Sg. Palum Tambun \& vicinity, $04^{\circ} 58^{\prime} \mathrm{N}$, $117^{\circ} 48^{\prime} \mathrm{E}, 150 \mathrm{~m}, 19-26 . i i i .1987$, J. Huisman (UMSP000107351) (RMNH).

Paratypes. Malaysia, Sarawak, Annah Rais. Sg. Semadang, 800 m, 27.x.2003, W. Mey, $10^{\star}$ (UMHU); Sabah, Crocker Range National Park, Sg. Myoog, 9.xi.2003, W. Mey, 9 ơ (UMHU); Kinabalu National Park, Poring Hot Springs, 30.x.2003, W. Mey, 20 (UMHU); Lohan near Ranau, Sg. Lohan, 7.xi.2003, W. Mey, 5 đo (UMHU); $60 \mathrm{~km}$ W of Lahad Datu, DVFC, brooklet above lotus pond, $04^{\circ} 58^{\prime} \mathrm{N}, 117^{\circ} 48^{\prime} \mathrm{E}, 150 \mathrm{~m}, 19-28 . x .1987$, Huisman \& de Jong, $1 \delta^{\Uparrow}(\mathrm{RMNH}) ; 12 \mathrm{~km} \mathrm{NNE}$ 

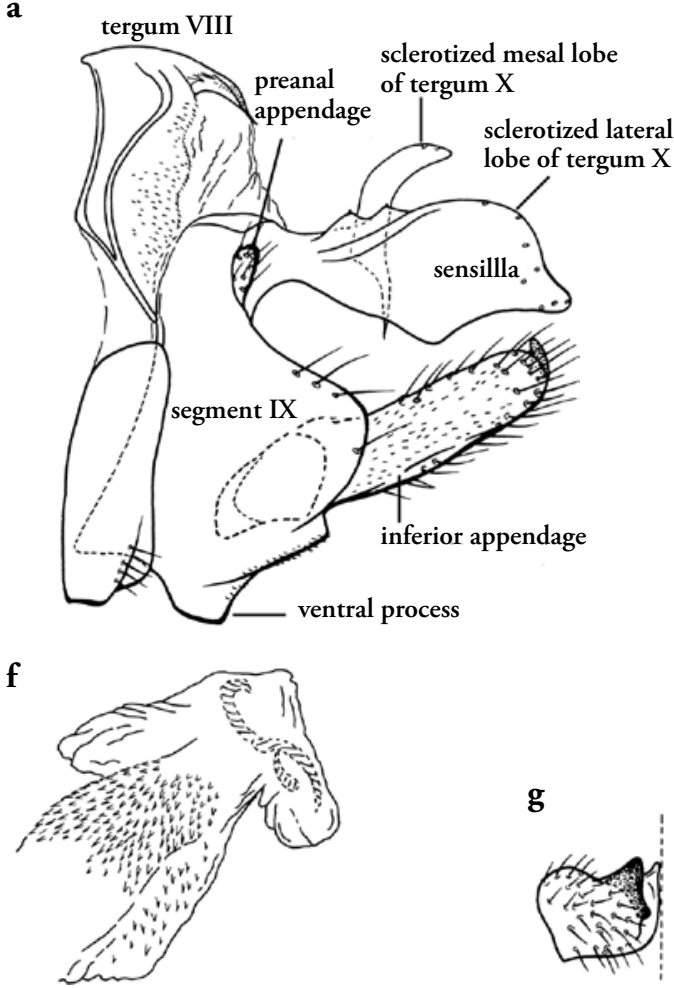

g

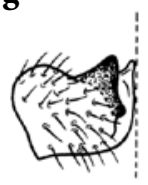

b
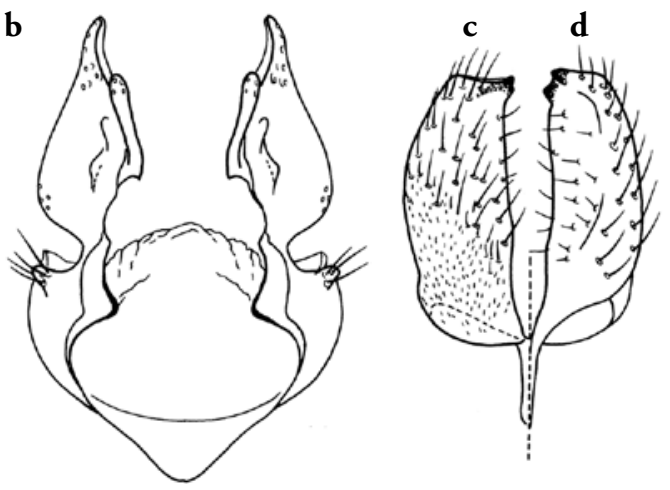

e

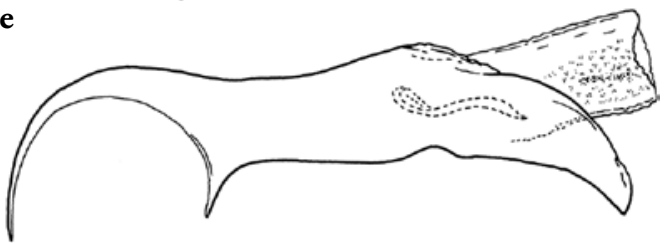

h

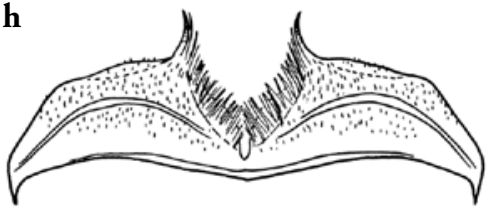

Fig. 15. Chimarra cuspidata, male genitalia. - a, lateral; b, segment IX and tergum X, dorsal; c, inferior appendage, ventral; $\mathrm{d}$, inferior appendage, dorsal; e, phallic apparatus, lateral; $f$, apex of expanded endotheca, lateral; g, inferior appendage, caudal; h, tergum VIII, dorsal.

Ranau, Poring Hot Spring, staff quarters, $06^{\circ} 03^{\prime} \mathrm{N}$, $116^{\circ} 42^{\prime} \mathrm{E}, 550 \mathrm{~m}$, 1.ii.1987, Huisman \& de Jong, $30^{\text {}}$ (UMSP); Kinabalu National Park, Poring, $570 \mathrm{~m}$, 8.ix.1983, Hevel \& Steiner, 20ิ, 5 ㅇ (NMNH).

Chimarra cuspidata is related to those species of the Chimarra tsudai group with a distinctly modified, mesally invaginated, spinose tergum VIII. In C. cuspidata, tergum VIII is relatively short, and the mesal invagination $\mathrm{V}$-shaped and margined by spines of a uniform length. It is easily diagnosed from any other members of this group by the shape of the lateral lobe of tergum X, which is distinctly inflated preapically, as viewed laterally, and by the distinctive apicomesal process on the inferior appendages, which is truncately cusped or weakly bicusped.

Adult. Color (in alcohol) dark brown. Length of forewing: male 4.3-4.9 mm. Forewing venation: stem of Rs curved, distinctly sclerotized and thickened at inflection; fork at base of discal cell somewhat thickened, nearly symmetrical, length of discal cell about 3 times width; $m$ crossvein proximal to crossveins $s$ and $r-m$, crossvein $s$ not hyaline; $2 \mathrm{~A}$ vein not intersecting 3A (apparently looped to 1A). Postocular parietal sclerite short. Maxillary palps relatively short, segment 3 distinctly longer than 2, 3 slightly longer than 5 . Protarsal claws of male enlarged, asymmetrical in shape, scarcely different in length.

Male genitalia. Abdominal segment VIII short; tergum about as long as sternum, modified, with V-shaped mesal excavation, excavation with short laciniate fringe; sternum VIII without posteroventral projection. Abdominal segment IX very short dorsolaterally, tergum obsolete dorsomesally, without distinct anterolateral apodemes; anteroventral margin in lateral view not, or scarcely produced; posterior margin sinuously produced; ventral process projecting ventrally, short, broad basally, subtriangular. Preanal appendage setose, very small, rounded. Inferior appendage about as long as tergum X; in lateral view straight, nearly uniformly wide, apex rounded; in ventral view nearly straight, apically with distinctly sclerotized, weakly bicuspidate projection. 

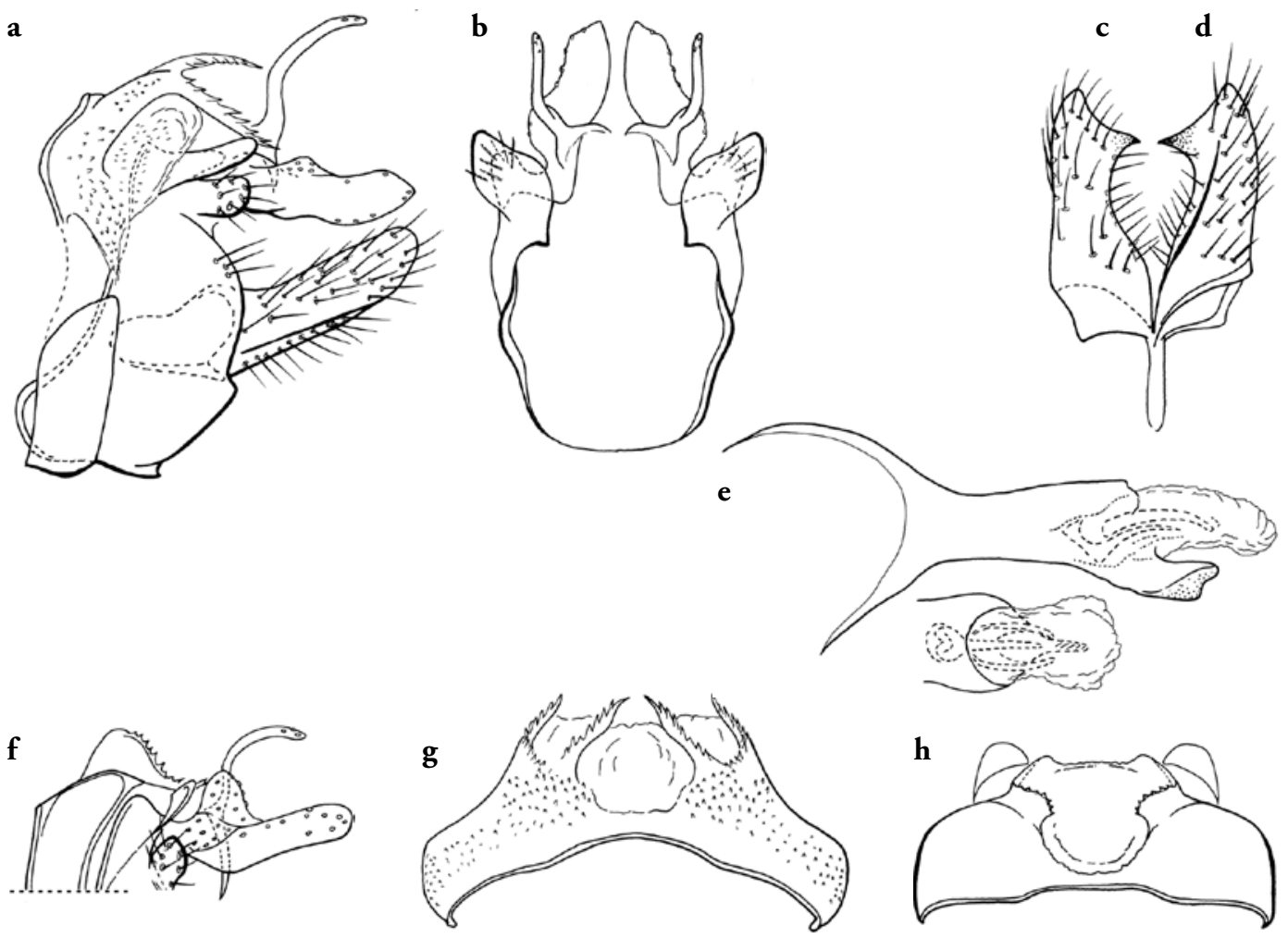

Fig. 16. Chimarra cygnus, male genitalia. - a, lateral; b, segment IX and tergum X, dorsal; c, inferior appendage, ventral; d, inferior appendage, dorsal; e, phallic apparatus, lateral; inset, apex of phallus, dorsal; $f$, variant form, detail of lateral; g, tergum VIII, dorsal; h, variant form, tergum VIII, dorsal.

Tergum X with sclerotized lateral lobes and separate mesal lobes, mesal membrane lacking; lateral lobe long, in lateral view inflated at midlength, apex narrow, bearing multiple sensilla; mesal lobe elongate, digitate, posterodorsally curved. Phallobase tubular, with pronounced basodorsal expansion, relatively short, ventral apex distinctly sclerotized, distinctly projecting, subacute, ventrally deflexed. Endotheca relatively short, with minutely spinulose region preapically, endothecal spines absent. Phallotremal sclerite complex composed of rod and ring structure, rod moderately elongate.

Etymology. This species is named Chimarra cuspidata, for the cusped apicomesal projections on the inferior appendage.

\section{Chimarra cygnus sp. $\mathbf{n}$.}

Fig. 16

Type material. Holotype: $\delta^{\dagger}$, Malaysia, Sabah, Kinabalu Park, Carson's Fall, 0602'N, 116³3'E, 1900 m, 7.xi.1987, Huisman \& de Jong (UMSP000107285) (RMNH).

Paratypes. Malaysia, Sabah, same data as holotype, 10 , 1 ㅇ (UMSP); Kinabalu National Park, Headquarters, Sg. Silau-Silau, 11.viii.2005, W. Mey, 2 §ै, 1 ㅇ (UMHU); Kinabalu National Park, tributary

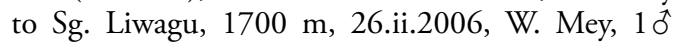
(UMHU); Kinabalu National Park, Headquarters, Sg. Tibabar, $06^{\circ} 02^{\prime} \mathrm{N}, 116^{\circ} 33^{\prime} \mathrm{E}, 1750 \mathrm{~m}, 12 . \mathrm{i} .1987$, J. Huisman, 1 tิ (RMNH); Kinabalu National Park, Headquarters, Sg. Liwagu, 1500 m, 10.viii.2005, W. Mey, $10^{\text {t }}$ (UMHU); Kinabalu National Park, Sungai Mesilau, 1800 m, 12.viii.2005, W. Mey, 110 , 39 (UMHU); Crocker Range, $5 \mathrm{~km} \mathrm{~N}$ Tenom, base of Sg. ulu Noloyan, $05^{\circ} 10^{\prime} \mathrm{N}, 115^{\circ} 56^{\prime} \mathrm{E}$, 1010, 10-11.x.1986, J. Huisman, 40 (RMNH); Kinabalu National Park, 12 km NNW Kundassang, Marei-Parei, trickle, $06^{\circ} 05^{\prime} \mathrm{N}, 116^{\circ} 31^{\prime} \mathrm{E}, 1670 \mathrm{~m}$, 9.iii.1987, J. Huisman, 40, 39 (UMSP).

Chimarra cygnus is related to those species of the Chimarra tsudai group with a modified, mesally 
invaginated, spinose tergum VIII. It is most readily diagnosed by the characteristic shape of the mesal lobes of tergum $\mathrm{X}$, which are elongate, digitate, and curved in a somewhat swan-neck like manner. Useful ancillary characters for diagnosing this species are the shape of the inferior appendage, which has a prominent, acute mesal projection preapically, and the shape of the lateral lobes of tergum X, each of which is moderately elongate and has a rounded basal projection. A distinctive variant of this species is illustrated in Figs $16 \mathrm{~F}$ and $16 \mathrm{H}$. The holotype is the form in Fig. 16A-E, G. Differences between these two forms include the spinose development of tergum VIII, the development of the ventral apex of the phallobase, and shape of the basal projection of the lateral lobe of tergum X. However, the overall differences are minor. We believe this variation to be intraspecific. Specimens from the Crocker Range were distinctly smaller in size and account for the wide variation in size found in this species. The closest relationship of $C$. cygnus is probably to C. lambi, sp. n. and C. danumensis, sp. n., both of which also have elongate phallotremal sclerites with accompanying dorsolateral sclerites. However, both of those species have the mesal projection of the inferior appendages very differently formed. The shape of the inferior appendages of $C$. cygnus is probably most similar to C. jannekae, sp. n. and $C$ caduca, sp. n., both of which differ significantly in the shape and orientation of the lateral and mesal lobes of tergum X.

Adult. Color (in alcohol) brown. Length of forewing: male 5.4-7.4 mm. Forewing venation: stem of Rs curved, slightly sclerotized and thickened at inflection; fork at base of discal cell distinctly thickened, nearly symmetrical, length of discal cell about 3 times width; $m$ crossvein proximal to crossveins $s$ and $r-m$, crossvein $s$ not hyaline; $2 \mathrm{~A}$ vein not intersecting $3 \mathrm{~A}$ (apparently looped to 1A). Postocular parietal sclerite moderately elongate. Maxillary palps elongate, segment 3 slightly greater than 2, 3 subequal to 5 . Protarsal claws of male enlarged, asymmetrical in shape and length.

Male genitalia. Abdominal segment VIII short; tergum about as long as sternum, modified, with mesal excavation, excavation bordered posterolaterally with short laciniate fringe, or sometimes an elongate, bifurcating process with marginal spines; sternum VIII without posteroventral projection. Abdominal segment IX relatively wide dorsolaterally, tergum obsolete dorsomesally, without distinct anterolateral apodemes; anteroventral margin in lateral view slightly produced, rounded; posterior margin sinuously produced; ventral process projecting ventrally, very short. Preanal appendage setose, small, rounded, slightly narrowed basally. Inferior appendage slightly longer than tergum X; in lateral view straight, nearly uniformly wide, apex rounded; in ventral view nearly straight, preapically with prominent, mesal, toothlike projection. Tergum $\mathrm{X}$ with sclerotized lateral lobes and separate mesal lobes, mesal membrane lacking; lateral lobe moderately long, in lateral view simple, rounded apically, with slight to distinct basodorsal projection, lobe bearing multiple sensilla; mesal lobe very elongate, narrow, digitate, strongly posterodorsally curved. Phallobase tubular, with pronounced basodorsal expansion, short, ventral apex distinctly sclerotized, distinctly projecting, forming laterally compressed process, variable in shape. Endotheca relatively short, granularly textured region not discernable; without endothecal spines. Phallotremal sclerite complex composed of rod and ring structure, rod moderately elongate, dorsolaterally, with pair of elongate curved sclerites.

Etymology. This species is called Chimarra cygnus, from the Latin cygnus, a swan, used as a noun in apposition, and referring to the swan-necked shape of the mesal processes of tergum $\mathrm{X}$ in this species.

\section{Chimarra danumensis sp. $\mathbf{n}$.}

Fig. 17

Type material. Holotype: $\widehat{\delta}$, Malaysia, Sabah, $60 \mathrm{~km}$ W Lahad Datu, Danum Valley Field Centre, Sg. Palum Tambun \& vicinity, $04^{\circ} 58^{\prime} \mathrm{N}$, $117^{\circ} 48^{\prime} \mathrm{E}, 150 \mathrm{~m}, 19-26.1 i i .1987$, J. Huisman (UMSP000107255) (RMNH).

Paratypes. Malaysia, Sabah, same data as holotype, 4 ơ (UMSP); Danum Valley Field Centre, Sg. Palun Tambun, 18.viii.2005, W. Mey, 30 (UMHU); $60 \mathrm{~km}$ W Lahad Datu, Danum Valley Field Centre, nr. Segama bridge, $04^{\circ} 58^{\prime} \mathrm{N}, 117^{\circ} 48^{\prime} \mathrm{E}, 150 \mathrm{~m}$, 20.x.1987, Huisman \& de Jong, 1 đ̊ (UMSP).

Chimarra danumensis is related to those species of the Chimarra tsudai group with a modified, mesally invaginated, spinose tergum VIII. The closest relationship of $C$. danumensis is probably to C. lambi, sp. n. and C. cygnus, sp. n, both of which also have an elongate phallotremal sclerite with accompanying dorsolateral sclerites. In $C$. danumensis this structure is very elongate, nearly the length of the phallobase. In overall features, $C$. danumensis is probably most similar to C. lambi, but can be easily distinguished by the diagnostic bicusped projection from the mesal surface of the inferior appendages, which in $C$. danumensis is located preapically rather than apically.

Adult. Color (in alcohol) brown. Length of forewing: male $4.6-5.0 \mathrm{~mm}$. Forewing venation: stem 
a

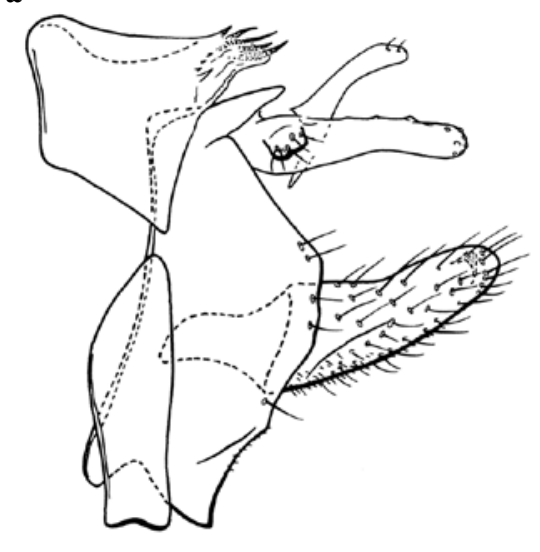

b

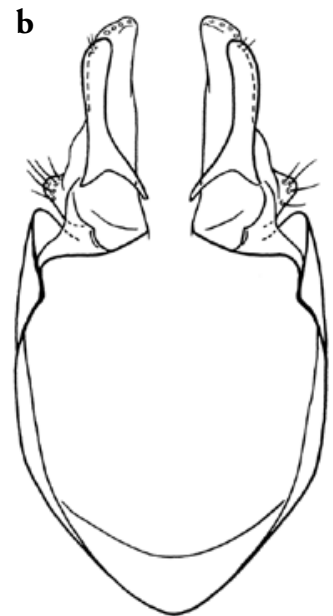

e
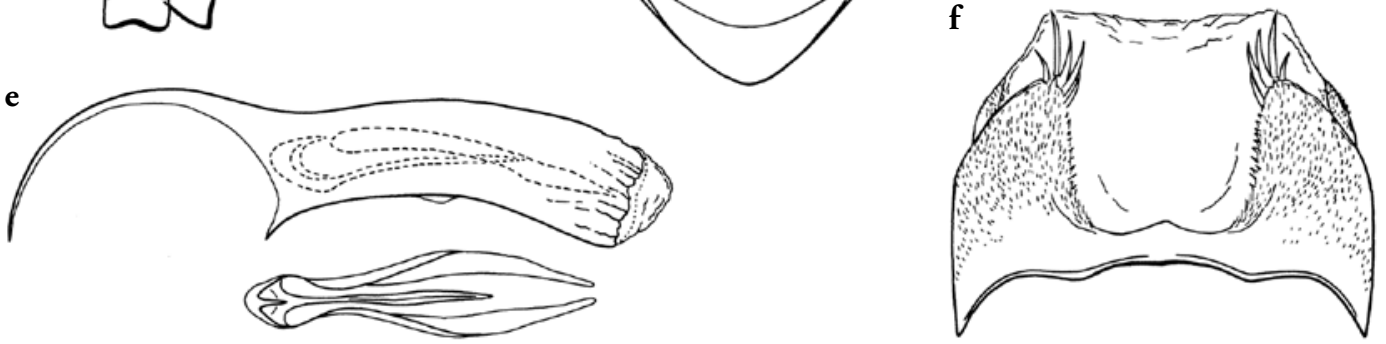

Fig. 17. Chimarra danumensis, male genitalia. - a, lateral; b, segment IX and tergum X, dorsal; c, inferior appendage, ventral; $d$, inferior appendage, dorsal; e, phallic apparatus, lateral; inset, phallotremal sclerite, dorsal; f, tergum VIII, dorsal.

of Rs curved, slightly sclerotized and thickened at inflection; fork at base of discal cell not thickened, nearly symmetrical, length of discal cell about 3 times width; $m$ crossvein proximal to crossveins $s$ and $r-m$, crossvein $s$ hyaline; $2 \mathrm{~A}$ vein not intersecting $3 \mathrm{~A}$ (apparently looped to 1A). Postocular parietal sclerite short. Maxillary palps relatively short, segment 3 slightly longer than 2, 3 subequal to 5 . Protarsal claws of male enlarged, asymmetrical in shape and length.

Male genitalia. Abdominal segment VIII short; tergum distinctly longer than sternum, modified, with broad, subquadrate mesal excavation, excavation apicolaterally with laciniate fringe; sternum VIII without posteroventral projection. Abdominal segment IX relatively wide dorsolaterally, tergum obsolete dorsomesally, without distinct anterolateral apodemes; anteroventral margin in lateral view not, or scarcely produced; posterior margin distinctly produced; ventral process projecting ventrally, broad basally, subtriangular. Preanal appendage setose, short, rounded, broadest basally. Inferior appendage about as long as tergum $\mathrm{X}$; in lateral view straight, nearly uniformly wide, apex rounded; in ventral view somewhat mesally curved, preapically with distinctly sclerotized, bicuspidate projection. Tergum X with sclerotized lateral lobes and separate mesal lobes, mesal membrane lacking; lateral lobe moderately long, in lateral view narrow, parallel sided, rounded apically, lobe bearing multiple sensilla; mesal lobe elongate, digitate, posterodorsally directed. Phallobase tubular, with pronounced basodorsal expansion, moderately elongate. Endotheca length not discernable (not expanded); without endothecal spines. Phallotremal sclerite complex composed of rod and ring structure, rod elongate, dorsolaterally, with pair of elongate curved sclerites.

Etymology. This species is named Chimarra danumensis after the Danum Valley Field Centre, where the holotype specimen was collected.

\section{Chimarra denticula sp. $\mathrm{n}$.}

Fig. 18

Type material. Holotype: $\widehat{\delta}$, Malaysia, Sabah, Crocker Range, $5 \mathrm{~km} \mathrm{~N}$ Tenom, base of $\mathrm{Sg}$. ulu Noloyan, $05^{\circ} 10^{\prime} \mathrm{N}, 115^{\circ} 56^{\prime} \mathrm{E}, 1010 \mathrm{~m}, 10-11 . x .1986$, J. Huisman (UMSP000107331) (RMNH).

Paratypes. Malaysia, Sabah, same data as holotype, 40 (UMSP). 


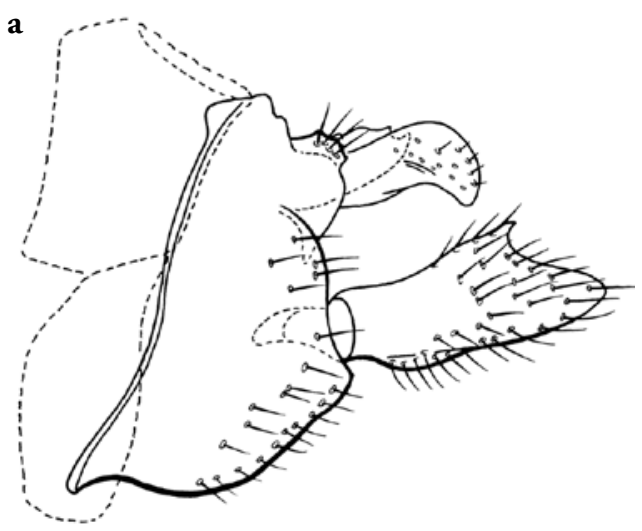

b

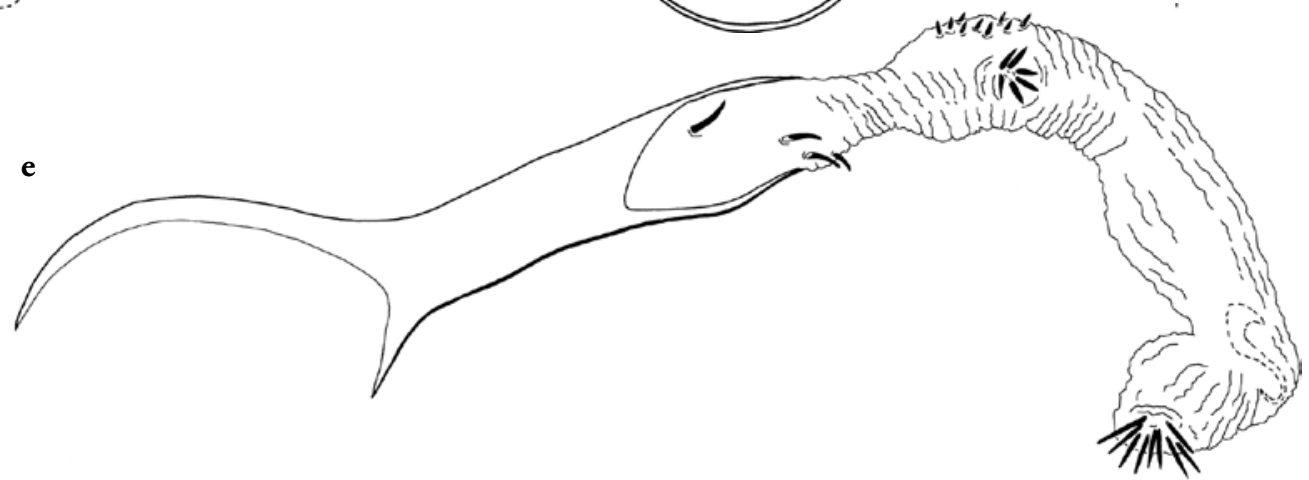

Fig. 18. Chimarra denticula, male genitalia. - a, lateral; b, segment IX and tergum $X$, dorsal; c, inferior appendage, ventral; $\mathrm{d}$, inferior appendage, dorsal; e, phallic apparatus, lateral.

Chimarra denticula resembles those species of the Chimarra tsudai group from Sabah without a modified tergum VIII, but with an acute projection or tooth on the dorsal margin of the inferior appendages, evident in lateral view (including C. karlijnae, sp. n., C. prokrustes Malicky, C. scolops, sp. n., C. sinitorum, sp. n., and C. stenodactylus, sp. n.). All of these species also have a phallus with numerous small spines and a narrow sclerotized dorsal extension, which extends over the basal part of the endotheca. They are additionally characterized by a mesal lobe of tergum X that is distinctly sclerotized and with an apex that is acute and dorsally curved. Among these species, C. denticula is most easily diagnosed by the short and distinctively shaped lateral and mesal lobes of tergum $\mathrm{X}$. It is also distinctive in having an additional short acute projection or tooth located preapically on the mesal surface of each inferior appendage. Chimarra stenodactylus also has short lateral and mesal lobes of tergum X, but these are differently shaped, and the base of the dorsal projection on the inferior appendage of this species is very narrow.

Adult. Color (in alcohol) yellowish-brown. Length of forewing: male $4.6-5.5 \mathrm{~mm}$. Forewing venation: stem of Rs curved, slightly sclerotized and thickened at inflection; fork at base of discal cell somewhat thickened, nearly symmetrical, length of discal cell about 3 times width; $m$ crossvein proximal to crossveins $s$ and $r-m$, crossvein $s$ not hyaline; $2 \mathrm{~A}$ vein not intersecting 3A (apparently looped to 1A). Postocular parietal sclerite moderately elongate. Maxillary palps relatively short, segment 3 slightly longer than 2, 2 subequal to 5. Protarsal claws of male enlarged, asymmetrical in shape and length.

Male genitalia. Abdominal segment VIII moderate in length; tergum longer than sternum, unmodified, entire; sternum VIII without posteroventral projection. Abdominal segment IX relatively wide dorsolaterally, tergum obsolete dorsomesally, without distinct anterolateral apodemes; anteroventral margin in lateral view not, or scarcely produced; posterior margin distinctly produced; ventral process absent or nearly so. Preanal appendage setose, very small, rounded. Inferior appendage longer than tergum X; in lateral view straight, nearly uniformly wide, apex rounded, dorsal margin with basally wide, acute projection at midlength; in ventral view somewhat mesally curved, mesal surface with small, preapical tooth-like 

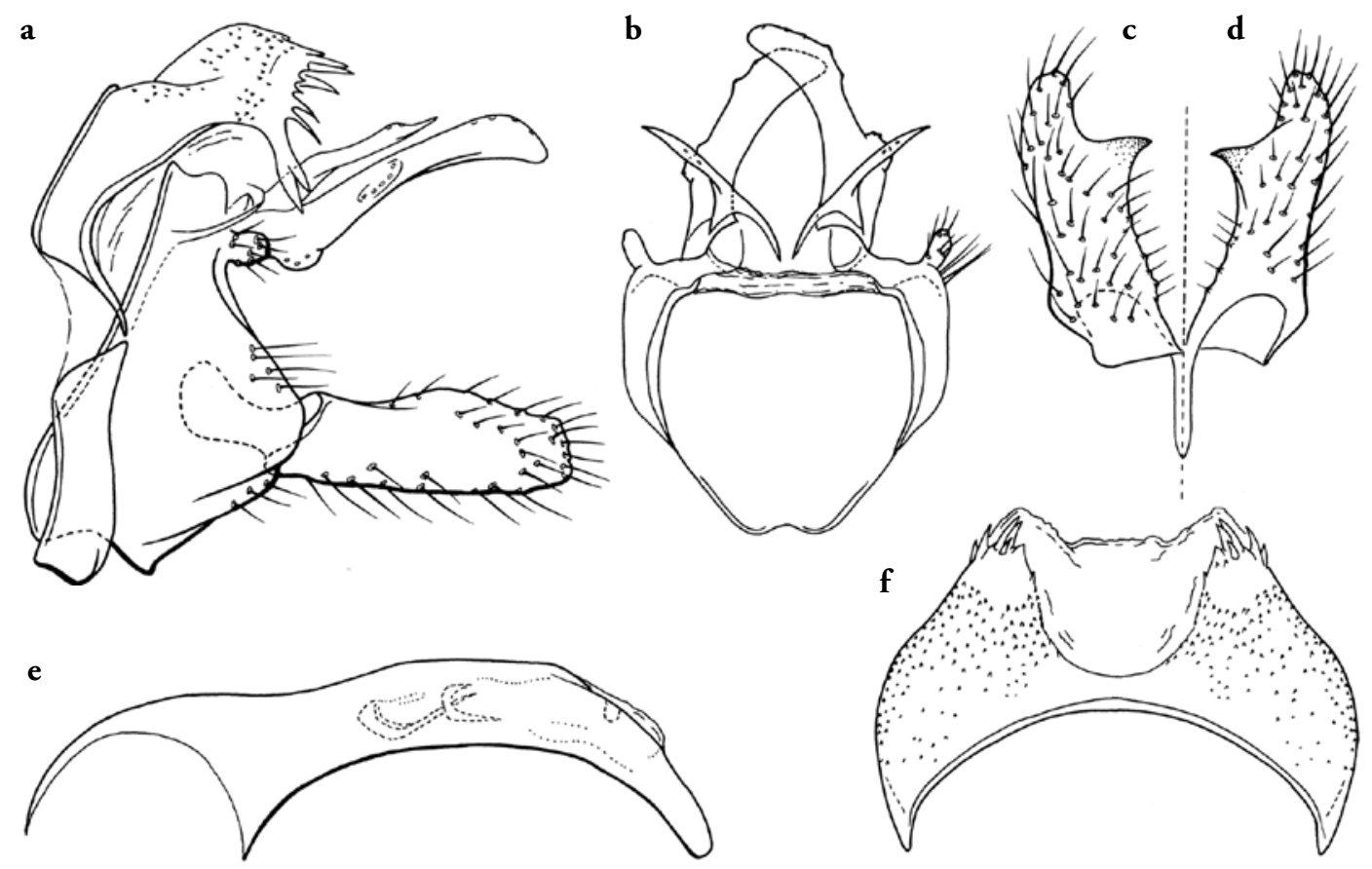

Fig. 19. Chimarra jannekae, male genitalia. - a, lateral; b, segment IX and tergum X, dorsal; c, inferior appendage, ventral; d, inferior appendage, dorsal; e, phallic apparatus, lateral; f, tergum VIII, dorsal.

projection. Tergum X with sclerotized lateral lobes and separate mesal lobes, mesal membrane lacking; lateral lobe short, in lateral view simple, rounded apically, distinctly downcurved, lobe bearing multiple sensilla; mesal lobe short, digitate, distinctly sclerotized, dorsally directed, apex with approximately 2 spine-like projections. Phallobase tubular, with pronounced basodorsal expansion, moderately elongate, relatively narrow, ventral apex lightly sclerotized, projecting, dorsal margin extended apically, elongate, narrow, lightly sclerotized. Endotheca elongate, tubular; with several tracks of small endothecal spines, basal track of few scattered spines, dorsal track of very short spines at midlength, lateral track of small spines at midlength, and apical track of slightly longer spines. Phallotremal sclerite complex composed of rod and ring structure, rod short.

Etymology. This species is named Chimarra denticula, as a diminutive form derived from the Latin word dens, a tooth, treated as a noun in apposition, and referring to the distinctive small preapical projection on the mesal surface of the inferior appendage in this species.

\section{Chimarra jannekae sp. $\mathbf{n}$.}

Figs 19, 41

Type material. Holotype: $\widehat{\delta}$, Malaysia, Sabah, Crocker Range, $40 \mathrm{~km} \mathrm{~S}$ of Kota Kinabalu, Sinsuron Rd., 1500, 20.xii.1989, J. Huisman, (UMSP000107161) (RMNH).

Paratypes. Malaysia, Sabah, same data as holotype, 7 đิ (UMSP); Kinabalu National Park, Sg. Liwagu, 1100 m, 12.viii.2005, W. Mey, 20 (UMHU); Kinabalu National Park, Sg. Liwagu, 1500 m, 10.viii.2005, W. Mey, 1 đิ (UMHU); Kinabalu National Park, tributary to Sg. Liwagu, $1200 \mathrm{~m}$, 13.viii.2005, W. Mey, $10^{\widehat{t}}$ (UMHU); Kinabalu National Park, Sg. Tibabar @ Liwagu trail, 06 $02^{\prime} \mathrm{N}, 116^{\circ} 33^{\prime} \mathrm{E}$, 1750 m, 2.x.1986, J. Huisman, $10^{\widehat{T}}$ (RMNH); Kinabalu National Park, Headquarters area, 1560 m, 13.ix.1983, Hevel \& Steiner, 20 (NMNH).

Chimarra jannekae belongs to a distinctive subgroup of the Chimarra tsudai group in which tergum VIII is highly modified, invaginated mesally and variously armed with spine-like projections. Among species from Sabah, it resembles C. caduca, sp. n and C. cygnus, 
a

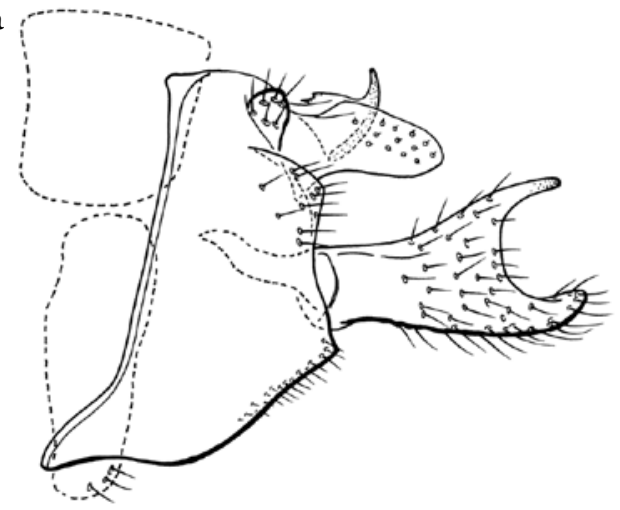

b

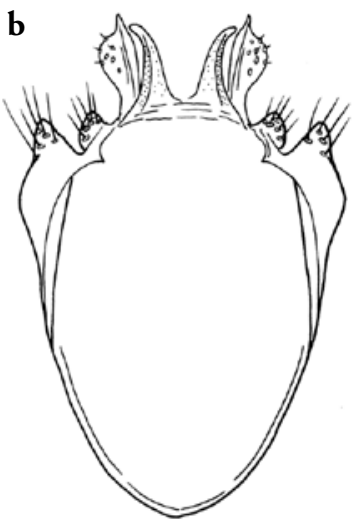

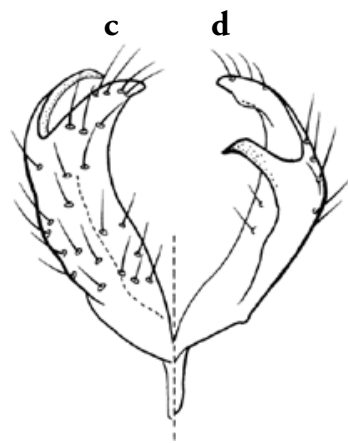

g

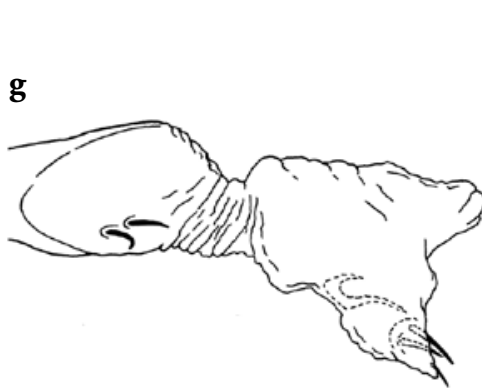

f
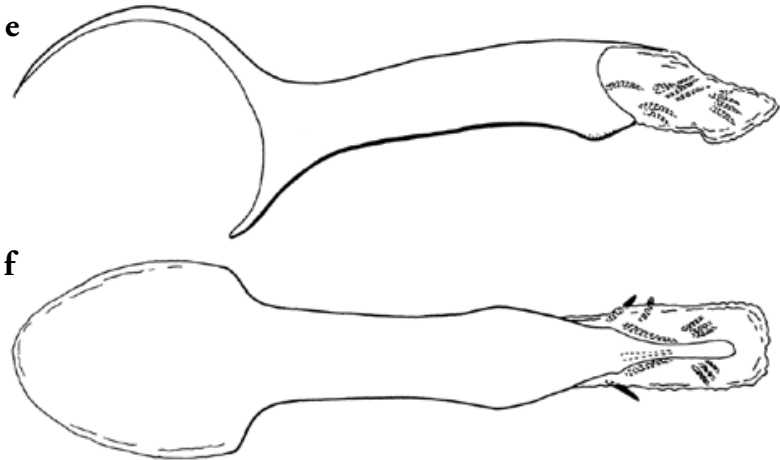

Fig. 20. Chimarra karlijnae, male genitalia. - a, lateral; b, segment IX and tergum X, dorsal; c, inferior appendage, ventral; $d$, inferior appendage, dorsal; e, phallic apparatus, lateral; f, phallic apparatus, dorsal; g, expanded endotheca, lateral.

sp. n. in the shape of the inferior appendages. Each inferior appendage in all of these species has a mesal spine-like process at just past midlength. Additionally, the phallic apparatus in all of these species lacks accessory spines. Diagnostic features of $C$. jannekae include the very elongate lateral lobes of tergum $\mathrm{X}$ and the elongate, downcurved and distinctly sclerotized apex of the phallobase.

Adult. Color (in alcohol) brown. Length of forewing: male $6.0-6.6 \mathrm{~mm}$. Forewing venation: stem of Rs curved, slightly sclerotized and thickened at inflection; fork at base of discal cell somewhat thickened, nearly symmetrical, length of discal cell about 3 times width; $m$ crossvein proximal to crossveins $s$ and $r-m$, crossvein $s$ not hyaline; $2 \mathrm{~A}$ vein not intersecting 3A (apparently looped to 1A). Postocular parietal sclerite moderately elongate. Maxillary palps moderately elongate, segment 3 slightly greater than 2, 3 subequal to 5 . Protarsal claws of male enlarged, asymmetrical in shape and length.

Male genitalia. Abdominal segment VIII short; tergum distinctly longer than sternum, modified, with broad, U-shaped mesal excavation, excavation apicolaterally with laciniate fringe, lateral margin forming downturned digitate projection. Preanal appendage setose, very small, rounded. Inferior appendage about as long as tergum X; in lateral view straight, nearly uniformly wide, apex rounded; in ventral view nearly straight, mesal surface with tooth-like projection just beyond midlength. Tergum $\mathrm{X}$ with sclerotized lateral lobes and separate mesal lobes, mesal membrane lacking; lateral lobe long, in lateral view narrow, apex slightly ventromesally curved, left and right lobes slightly asymmetri$\mathrm{cal}$; mesal lobe elongate, digitate, posterolaterally directed, apex acute. Phallobase tubular, with pronounced basodorsal expansion, moderately elongate, ventral apex distinctly sclerotized, distinctly projecting, subacute, strongly ventrally deflexed. Endotheca relatively short, with granularly textured region, without endothecal spines. Phallotremal sclerite complex composed of rod and ring structure, rod moderately elongate, with apicolateral sclerites.

Etymology. Named for Janneke Holzenthal, youngest daughter of J. Huisman and R. Holzenthal. 


\section{Chimarra karlijnae sp. n.}

Fig. 20

Type material. Holotype: $\widehat{\widehat{A}}$, Malaysia, Sabah, $12 \mathrm{~km}$ NNE Ranau, Poring Hot Spring, Sg. Kepungit II, $06^{\circ} 04^{\prime} \mathrm{N}, 116^{\circ} 41^{\prime} \mathrm{E}, 700 \mathrm{~m}, 26.1 .1987$, J. Huisman (UMSP000107256) (RMNH).

Paratypes. Malaysia, Sabah, same data as holotype, 5 ㅇ (UMSP); Crocker Range, $5 \mathrm{~km} \mathrm{~N}$ Tenom, base of Sg. ulu Noloyan, $05^{\circ} 10^{\prime} \mathrm{N}, 115^{\circ} 56^{\prime} \mathrm{E}, 1010 \mathrm{~m}$, 10-11.x.1986, J. Huisman, $10^{\star}$ (RMNH); Poring Hot Spring, 12 km NNE Ranau, Sg. Kepungit waterfall, $06^{\circ} 03^{\prime} \mathrm{N}, 116^{\circ} 42^{\prime} \mathrm{E}, 550 \mathrm{~m}$, 4.xii.1986, J. Huisman, $10^{\hat{\sigma}}$ (UMSP); $18 \mathrm{~km}$ on road Keningau-Kimanis, gravel quarry, $05^{\circ} 26^{\prime} \mathrm{N}, 116^{\circ} 05^{\prime} \mathrm{E}$, 1050 m, 20.i.1987, J. Huisman, 30ิ (UMSP); $12 \mathrm{~km}$ NNE Ranau, Poring Hot Spring, Sg. Tananansad, $06^{\circ} 03^{\prime} \mathrm{N}, 116^{\circ} 42^{\prime} \mathrm{E}, 560 \mathrm{~m}$, 28.viii.1986, J. Huisman, $10^{\hat{0}}(\mathrm{RMNH})$; Poring Hot Spring, $12 \mathrm{~km}$ NNE Ranau, Sg. Kipogoh, 06 03'N, $116^{\circ} 42^{\prime} \mathrm{E}, 550 \mathrm{~m}$, 9.xii.1986, J. Huisman, 30 (UMSP); 12 km NNE Ranau, Poring Hot Spring, staff quarters, $06^{\circ} 03^{\prime} \mathrm{N}, 116^{\circ} 42^{\prime} \mathrm{E}, 550 \mathrm{~m}$, 1.ii.1987, Huisman \& de Jong, $1 \overbrace{}^{\star}(\mathrm{RMNH})$.

Chimarra karlijnae resembles those species of the Chimarra tsudai group from Sabah that lack a modified tergum VIII and have an acute projection or tooth on the dorsal margin of the inferior appendages, evident in lateral view (including $C$. denticula, sp. n., C. prokrustes Malicky, C. scolops, sp. n., C. sinitorum, sp. n., and C. stenodactylus, sp. n.). All of these species also have a phallus with numerous small spines and a narrow sclerotized dorsal extension that extends over the basal part of the endotheca. They are additionally characterized by a mesal lobe of tergum X that is distinctly sclerotized and with an apex that is acute and dorsally curved. Among these species, $C$. karlijnae is easily diagnosed by the shape of the inferior appendage, which has the dorsal process enlarged, posteriorly directed and subequal in length and development to the ventral margin, thus producing a distinctive $\mathrm{C}$-shaped apex to the inferior appendage.

Adult. Color (in alcohol) light brown. Length of forewing: male $4.9-5.6 \mathrm{~mm}$, female $5.0-5.8 \mathrm{~mm}$. Forewing venation: stem of Rs curved, slightly sclerotized and thickened at inflection; fork at base of discal cell somewhat thickened, nearly symmetrical, length of discal cell about 4 times width; $m$ crossvein proximal to crossveins $s$ and $r-m$, crossvein $s$ not hyaline; $2 \mathrm{~A}$ vein not intersecting 3A (apparently looped to $1 \mathrm{~A})$. Postocular parietal sclerite moderately elongate. Maxillary palps moderately elongate, segment 3 slightly greater than 2, 3 subequal to 5 . Protarsal claws of male enlarged, asymmetrical in shape and length.

Male genitalia. Abdominal segment VIII moderate in length; tergum longer than sternum, unmodified, with V-shaped mesal depression; sternum VIII without posteroventral projection. Abdominal segment IX relatively wide dorsolaterally, tergum obsolete dorsomesally, without distinct anterolateral apodemes; anteroventral margin in lateral view slightly produced; posterior margin slightly produced; ventral process absent or nearly so. Preanal appendage setose, very small, rounded. Inferior appendage much longer than tergum $\mathrm{X}$; in lateral view straight, widest mesally, narrow distally, apex rounded, dorsal margin with very basally wide, apically acute projection at midlength, the projection directed posteriorly and imparting a crescentic appearance to apex of inferior appendage; in ventral view somewhat mesally curved, mesal surface without processes. Tergum X with sclerotized lateral lobes and separate mesal lobes, mesal membrane lacking; lateral lobe short, in lateral view simple, rounded apically, lobe bearing multiple sensilla; mesal lobe short, digitate, distinctly sclerotized, dorsally directed, with basal sensillate lobe and dorsally hooked apical projection. Phallobase tubular, with pronounced basodorsal expansion, moderately elongate, relatively narrow, ventral apex lightly sclerotized, slightly projecting, dorsal margin extended apically, elongate, narrow, lightly sclerotized. Endotheca relatively short, with basal tract of short spines and also apicoventral tract of short spines. Phallotremal sclerite complex composed of rod and ring structure, rod relatively short. Etymology. Named for Karlijn Holzenthal, oldest daughter of J. Huisman and R. Holzenthal.

\section{Chimarra kinabaluensis sp. $\mathbf{n}$.}

Fig. 21

Type material. Holotype: $\delta$, Malaysia, Sabah, Kinabalu National Park, Headquarters, confluence Sg. Liwagu \& Sg. Silau-Silau, $06^{\circ} 00^{\prime} \mathrm{N}, 116^{\circ} 33^{\prime} \mathrm{E}$, 1450 m, 27.vii.1986, J.Huisman(UMSP000107146) (RMNH).

Paratypes. Malaysia, Sabah, Kinabalu National Park, Headquarters, Sg. Liwagu @ Silau-Silau trail, $06^{\circ} 00^{\prime} \mathrm{N}, 116^{\circ} 33^{\prime} \mathrm{E}, 1470 \mathrm{~m}, 24 . v i i i .1986$, J. Huisman, 1 ô (UMSP); same locality, 10.i.1987, J. Huisman, $1 \delta^{\hat{0}}(\mathrm{RMNH})$; Kundassang, Sg. Mesilau, 1st bridge after golf course, $06^{\circ} 01^{\prime} \mathrm{N}, 116^{\circ} 36^{\prime} \mathrm{E}, 1650 \mathrm{~m}$, 3.x.1986, J. Huisman, 20 (UMSP).

Chimarra kinabaluensis is another species belonging to the subgroup of the Chimarra tsudai group in which 

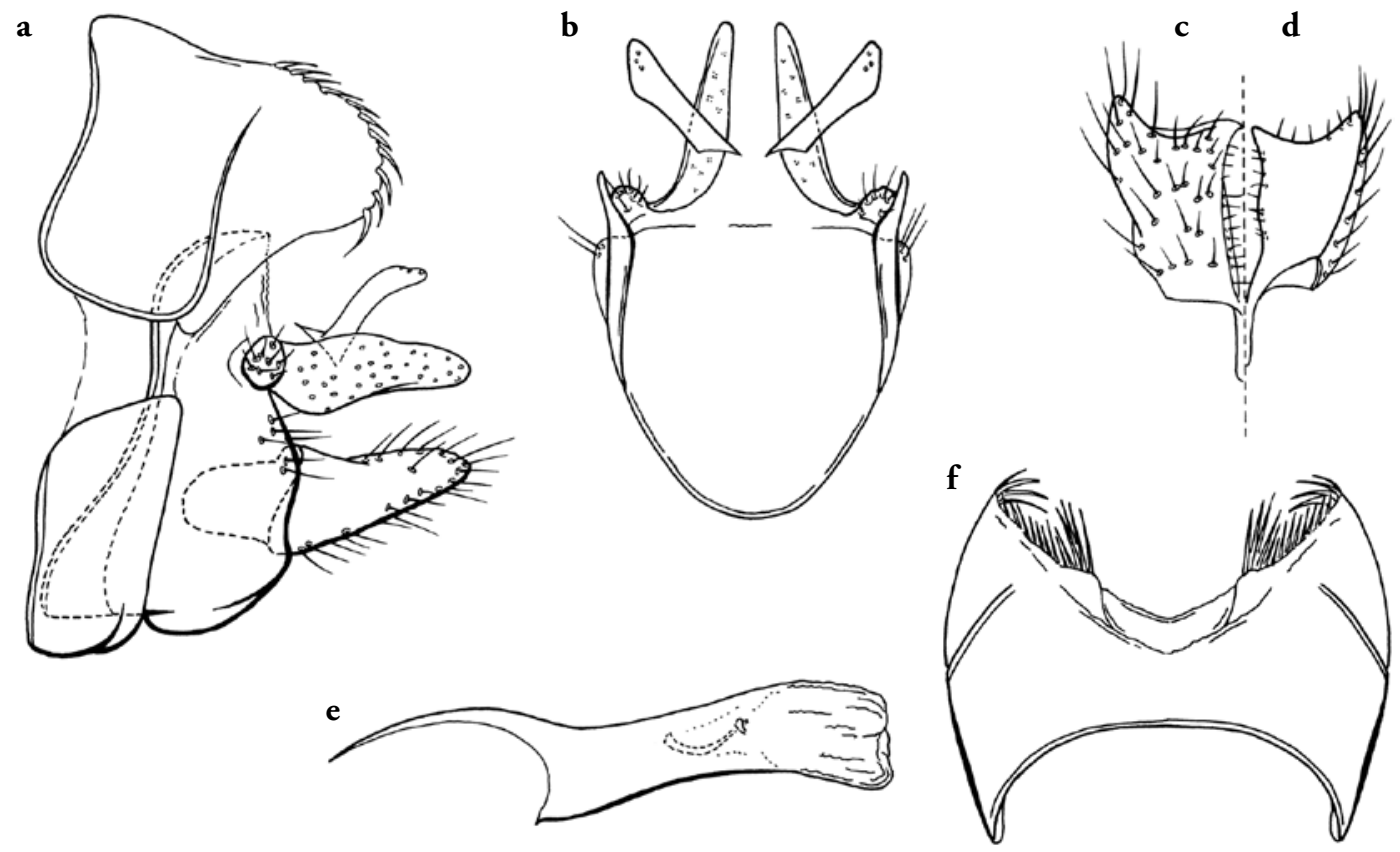

Fig. 21. Chimarra kinabaluensis, male genitalia. - a, lateral; b, segment IX and tergum X, dorsal; c, inferior appendage, ventral; d, inferior appendage, dorsal; e, phallic apparatus, lateral; f, tergum VIII, dorsal.

tergum VIII is highly modified, invaginated mesally and variously armed with spine-like projections. It is perhaps most similar to $C$. preapicalis, sp. n., which has a similarly shaped segment IX. The most useful diagnostic character is the shape of the inferior appendages, which are very short, and possess an acute mesal projection just preapically on the mesal margin. The greatly projecting tergum VIII, bordered by uniformly sized spines is also a useful diagnostic character. Other features it possesses a phallotremal sclerite that is not greatly enlarged, and an endotheca that is relatively short and lacks distinctive spines.

Adult. Color (in alcohol) light brown. Length of forewing: male 5.0-6.4 mm. Forewing venation: stem of Rs curved, slightly sclerotized and thickened at inflection; fork at base of discal cell not thickened, nearly symmetrical, length of discal cell about 3 times width; $m$ crossvein somewhat proximal to crossveins $s$ and $r-m$, crossvein $s$ not hyaline; $2 \mathrm{~A}$ vein not intersecting 3A (apparently looped to $1 \mathrm{~A}$ ). Postocular parietal sclerite moderately elongate. Maxillary palps elongate, segment 3 subequal to 2 and 5 . Protarsal claws of male enlarged, asymmetrical in shape and length.

Male genitalia. Abdominal segment VIII moderate in length; tergum distinctly longer than sternum, modified, with V-shaped mesal excavation, excavation bordered posterolaterally by spine-like lac- iniate fringe; sternum VIII without posteroventral projection. Abdominal segment IX relatively wide dorsolaterally, tergum obsolete dorsomesally, without distinct anterolateral apodemes; anteroventral margin in lateral view slightly produced; posterior margin slightly produced; ventral process projecting ventrally, very short, broad basally, broadly rounded. Preanal appendage setose, very small, rounded. Inferior appendage short, about as long as tergum $\mathrm{X}$; in lateral view straight, widest basally, gradually narrowing distally, apex rounded; in ventral view nearly straight, preapically with very prominent, basally broad, ventromesal, tooth-like projection. Tergum $\mathrm{X}$ with sclerotized lateral lobes and separate mesal lobes, mesal membrane lacking; lateral lobe moderately long, in lateral view simple, rounded apically, lobe bearing multiple sensilla; mesal lobe elongate, digitate, slightly posterodorsally curved. Phallobase tubular, with pronounced basodorsal expansion, short. Endotheca relatively short, granularly textured region not discernable; without endothecal spines. Phallotremal sclerite complex composed of elongate ring, rod very reduced, preapically with short lateral sclerites.

Etymology. This species is named Chimarra kinabaluensis for Kinabalu National Park, where the holotype specimen was collected. 
a
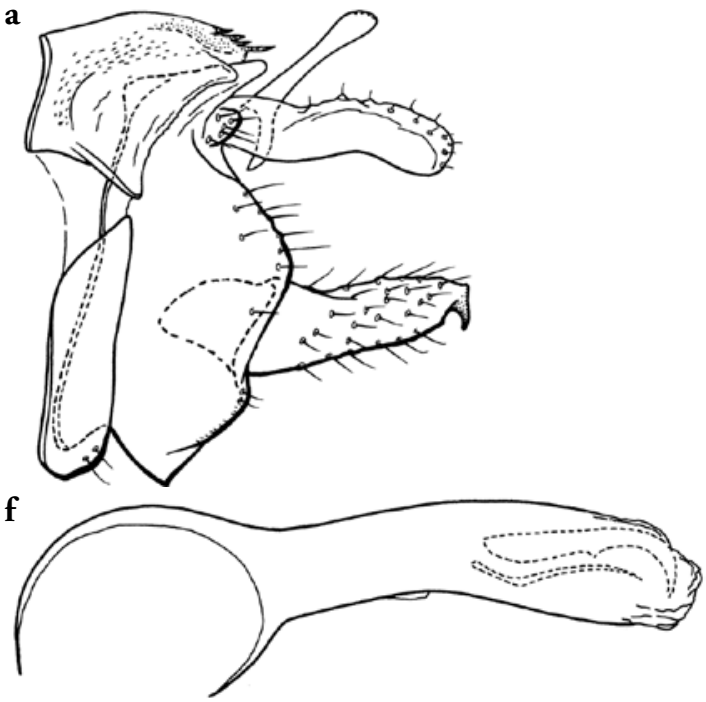

g

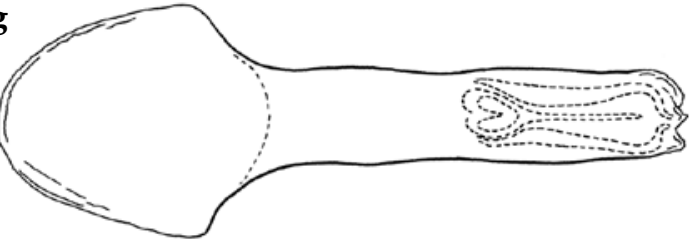

b

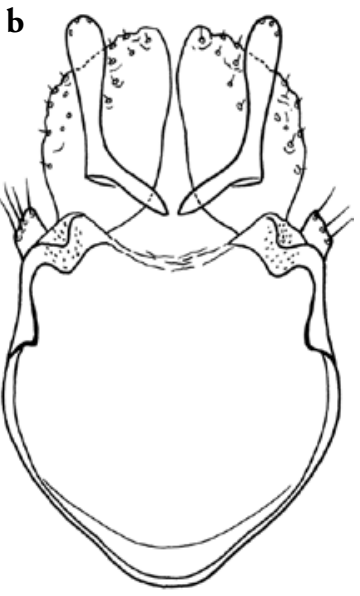

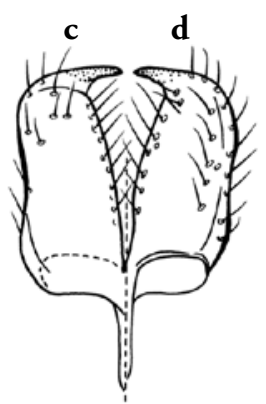

e

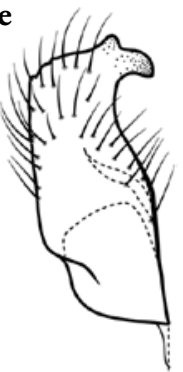

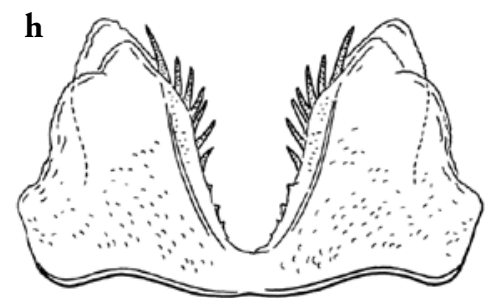

Fig. 22. Chimarra lambi, male genitalia. - a, lateral; b, segment IX and tergum $X$, dorsal; c, inferior appendage, ventral; $d$, inferior appendage, dorsal; e, inferior appendage, oblique caudal; $f$, phallic apparatus, lateral; $g$, phallic apparatus, dorsal; h, tergum VIII, dorsal.

\section{Chimarra lambi sp. n.}

Fig. 22

Type material. Holotype: $\widehat{\delta}$, Malaysia, Sabah, Poring Hot Spring, $12 \mathrm{~km}$ NNE Ranau, Sg. Kipogoh, $06^{\circ} 03^{\prime} \mathrm{N}, 116^{\circ} 42^{\prime} \mathrm{E}, 550 \mathrm{~m}$, 1.ii.1987, J. Huisman (UMSP000107253) (RMNH).

Paratypes. Malaysia, Sabah, SW Sabah, nr. Long Pa Sia (west), 1050 m, 25.xi-8.xii.1987, Achterberg, 1 추 (RMNH); 10 km S Long Pa Sia, Sg. Malabit, $04^{\circ} 21^{\prime} \mathrm{N}, 115^{\circ} 41^{\prime} \mathrm{E}, 1180 \mathrm{~m}$, 2.xii.1987, Huisman \& Achterberg, $2 \sigma^{\top}(\mathrm{RMNH}) ; 18 \mathrm{~km}$ on road Keningau-Kimanis, gravel quarry, $05^{\circ} 26^{\prime} \mathrm{N}, 116^{\circ} 05^{\prime} \mathrm{E}$, 1050 m, 20.i.1987, J. Huisman, 2 क , 49 (UMSP); $12 \mathrm{~km}$ NNE Ranau, Poring Hot Spring, Sg. Tananansad, $06^{\circ} 03^{\prime} \mathrm{N}, 116^{\circ} 42^{\prime} \mathrm{E}, 560 \mathrm{~m}$, 28.viii.1986, J. Huisman, 40ิ (UMSP); $2 \mathrm{~km}$ SW Long Pa Sia, Long Rurun (Sg. Ritan), $04^{\circ} 23^{\prime} \mathrm{N}, 115^{\circ} 42^{\prime} \mathrm{E}$, 1040 m, 5.xii.1987, Huisman \& Achterberg, $20^{\top}$ (RMNH); Poring Hot Spring, $12 \mathrm{~km}$ NNE Ranau,

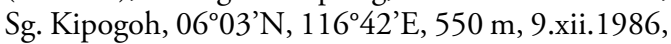
J. Huisman, $2 \sigma^{\star}$ (UMSP); 12 km NNE Ranau, Poring Hot Spring, staff quarters, $06^{\circ} 03^{\prime} \mathrm{N}, 116^{\circ} 42^{\prime} \mathrm{E}$,

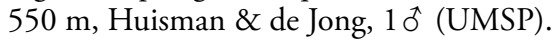

Chimarra lambi is another species belonging to the distinctive subgroup of the Chimarra tsudai group in which tergum VIII is deeply invaginated mesally and variously armed with spine-like projections. It is related to subgroup within this latter group in which the phallotremal sclerite complex is greatly enlarged and sclerotized and has elongate dorsolateral sclerites. Other species from Sabah having this combination of characters include C. cygnus, sp. n. and $C$. danumensis, sp. n. Chimarra lambi is most similar to $C$. danumensis, especially in having inferior appendages with the apex bicusped, but differs diagnostically in that the cusps are located more apically, and also in that the lateral lobes of tergum $\mathrm{X}$ are more dorsoventrally flattened and the phallotremal sclerite complex is shorter.

Adult. Color (in alcohol) light brown. Length of forewing: male $5.0-5.6 \mathrm{~mm}$, female $5.2-5.8 \mathrm{~mm}$. Forewing venation: stem of Rs curved, slightly sclerotized and thickened at inflection; fork at base of discal cell somewhat thickened, nearly symmetrical, length of discal cell about 3 times width; $m$ crossvein proximal to crossveins $s$ and $r-m$, crossvein $s$ not hyaline; $2 \mathrm{~A}$ vein not intersecting 3A (apparently looped 

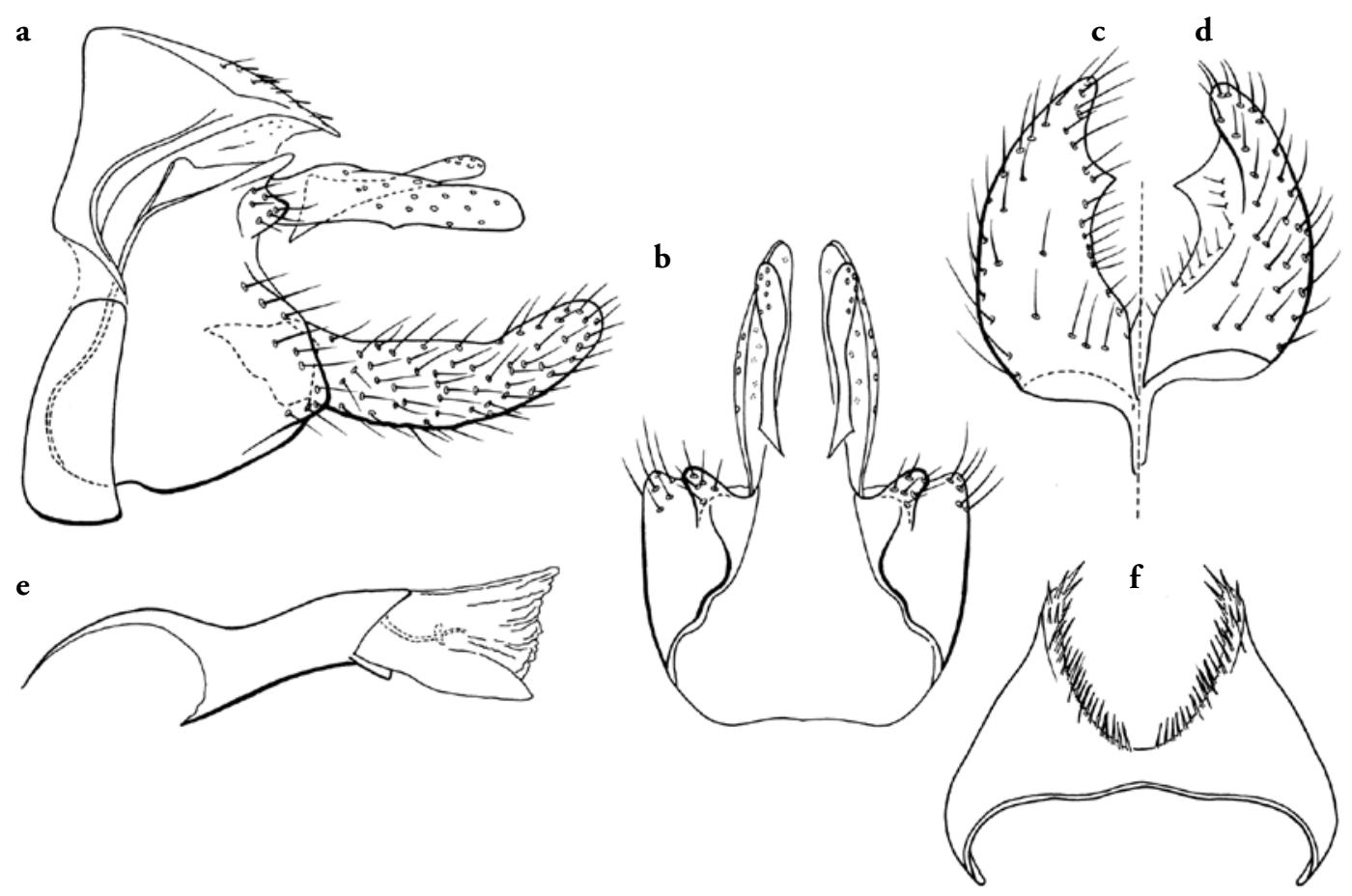

Fig. 23. Chimarra liwaguensis, male genitalia. - a, lateral; b, segment IX and tergum X, dorsal; c, inferior appendage, ventral; $\mathrm{d}$, inferior appendage, dorsal; e, phallic apparatus, lateral; f, tergum VIII, dorsal.

to 1 A). Postocular parietal sclerite short. Maxillary palps moderately elongate, segment 3 slightly greater than 2, 3 subequal to 5 . Protarsal claws of male enlarged, asymmetrical in shape and length.

Male genitalia. Abdominal segment VIII moderate in length; tergum distinctly longer than sternum, modified, with $\mathrm{V}$-shaped mesal excavation, excavation apicolaterally with laciniate fringe; sternum VIII without posteroventral projection. Abdominal segment IX relatively wide dorsolaterally, tergum obsolete dorsomesally, without distinct anterolateral apodemes; anteroventral margin in lateral view not, or scarcely produced; posterior margin distinctly produced; ventral process projecting ventrally, short, broad basally, subtriangular. Preanal appendage setose, short, rounded, broadest basally. Inferior appendage about as long as tergum $\mathrm{X}$; in lateral view straight, nearly uniformly wide, apex with mesally directed process; in ventral view nearly straight, apex subtruncate; in caudal view with evident sclerotized, bicusped projection. Tergum $\mathrm{X}$ with sclerotized lateral lobes and separate mesal lobes, mesal membrane lacking; lateral lobe moderately long, with apex broadly rounded, in lateral view simple, dorsoventrally flattened, rounded apically, lobe bearing multiple short setae or seta-like sensilla; mesal lobe elongate, digitate, posterodorsally directed. Phallobase tubular, with pronounced basodorsal expansion, moderately elongate. Endotheca length not discernable (not expanded); without endothecal spines. Phallotremal sclerite complex composed of rod and ring structure, rod elongate, dorsolaterally, with pair of elongate curved sclerites.

Etymology. Named for Anthony Lamb, friend of J. Huisman.

\section{Chimarra liwaguensis sp. $\mathbf{n}$.}

Fig. 23

Type material. Holotype: $\widehat{\sigma}$, Malaysia, Sabah, Kinabalu National Park, Headquarters, Sg. Liwagu crossing Silau-Silau trail, $06^{\circ} 00^{\prime} \mathrm{N}, 116^{\circ} 33^{\prime} \mathrm{E}, 1470 \mathrm{~m}$, 15.viii.1986, J. Huisman (UMSP000107130) (RMNH).

Paratypes. Malaysia, Sabah, same data as holotype, 3 ㅇ (UMSP); Kinabalu National Park, Sg. Liwagu, 1100 m, 12.viii.2005, W. Mey, $30 \hat{~(M N H U) ; ~}$ Kinabalu National Park, Headquarters, Sg. Liwagu @ Silau-Silau trail, 06 $00^{\prime} \mathrm{N}, 116^{\circ} 33^{\prime} \mathrm{E}, 1470 \mathrm{~m}$, 22.i.1987, Huisman \& de Jong, $20^{\uparrow}$ (UMSP); same locality, 10.ii.1987, J. Huisman, 10 , 16 (UMSP); 
same locality, 15.xi.1986, J. Huisman, 10 , $7 q$ (RMNH); Kinabalu National Park, Headquarters, on bridge to “Sg. Liwagu section I", $06^{\circ} 00^{\prime} \mathrm{N}$, $116^{\circ} 33^{\prime} \mathrm{E}, 1480 \mathrm{~m}$, 9.ii.1987, J. Huisman, 1 o 1 , 1 ㅇ (RMNH); Kundassang, Sg. Mesilau, 1st bridge after golf course, $06^{\circ} 01^{\prime} \mathrm{N}, 116^{\circ} 36^{\prime} \mathrm{E}, 1650 \mathrm{~m}, 3 . x .1986$, J. Huisman, 7§, 29 (UMSP); Kinabalu National Park, Headquarters area, 1560 m, 13.ix.1983, Hevel \& Steiner, 1 ô (NMNH).

Chimarra liwaguensis is another species of the Chimarra tsudai group in which tergum VIII is deeply invaginated mesally and variously armed with spinelike projections. It is easily diagnosed by the shape of the inferior appendage, which, in ventral view, is distinctly mesally curved, broad basally, narrow and rounded apically, and has an acute projection just past midlength from the ventral surface. The posteromesal excavation of tergum is also a useful diagnostic character in that it is deep and U-shaped and margined by spines of a uniform length. Additionally, the phallic apparatus is simple in structure, with a very short phallobase and without internal spines. Adult. Color (in alcohol) brown. Length of forewing: male $6.3-7.0 \mathrm{~mm}$, female $6.7-7.2 \mathrm{~mm}$. Forewing venation: stem of Rs curved, slightly sclerotized and thickened at inflection; fork at base of discal cell somewhat thickened, nearly symmetrical, length of discal cell about 3 times width; $m$ crossvein proximal to crossveins $s$ and $r-m$, crossvein $s$ not hyaline; $2 \mathrm{~A}$ vein not intersecting $3 \mathrm{~A}$ (apparently looped to 1A). Postocular parietal sclerite elongate. Maxillary palps moderately elongate, segment 3 subequal to 2 , 5 very narrow, slightly shorter than 3 . Protarsal claws of male enlarged, asymmetrical in shape and length. Male genitalia. Abdominal segment VIII moderate in length; tergum distinctly longer than sternum, modified, with broad, U-shaped mesal excavation, excavation with short laciniate fringe; sternum VIII without posteroventral projection. Abdominal segment IX relatively wide dorsolaterally, tergum obsolete dorsomesally, without distinct anterolateral apodemes; anteroventral margin in lateral view slightly produced, rounded; posterior margin distinctly produced; ventral process absent or nearly so. Preanal appendage setose, very small, rounded. Inferior appendage longer than tergum $\mathrm{X}$; in lateral view slightly curved, nearly uniformly wide, apex broadly rounded; in ventral view strongly mesally curved, broad basally, ventromesally with broad, apically acute projection. Tergum X with sclerotized lateral lobes and separate mesal lobes, mesal membrane lacking; lateral lobe moderately long, with apex broadly rounded, in lateral view narrow, parallel sided, rounded apically, lobe bearing multiple sensilla; mesal lobe elongate, digitate, reclinate, posteriorly directed. Phallobase tubular, with pronounced basodorsal expansion, short. Endotheca relatively short; without endothecal spines. Phallotremal sclerite complex composed of rod and ring structure, rod short, preapically with short lateral sclerites.

Etymology. This species is named Chimarra liwaguensis for the stream, Sungai Liwagu, where the holotype specimen was collected.

\section{Chimarra noloyan sp. $\mathbf{n}$.}

Fig. 24

Type material. Holotype: $\widehat{\delta}$, Malaysia, Sabah, Crocker Range, $5 \mathrm{~km} \mathrm{~N}$ Tenom, base of $\mathrm{Sg}$. ulu Noloyan, $05^{\circ} 10^{\prime} \mathrm{N}, 115^{\circ} 56^{\prime} \mathrm{E}, 1010 \mathrm{~m}, 10-11 . x .1986$, J. Huisman (UMSP000107155) (RMNH).

Paratypes. Malaysia, Sabah, Kinabalu National Park, Sungai Liwagu, 1100 m, 12.viii.2005, W. Mey, 40ે, 3 ㅇMU).

Chimarra noloyan also belongs to the distinctive subgroup of the Chimarra tsudai group in which tergum VIII is invaginated mesally and variously armed with spine-like projections. Generalized features it possesses, in common with some other members of this group, are a phallotremal sclerite that is not greatly enlarged, and an endotheca that is relatively short and lacks distinctive spines. In $C$. noloyan the length of tergum VIII is short, thus the mesal invagination is not very deep and the posterior fringe of spinelike projections are irregular in length and are not formed into elongate processes. Chimarra noloyan is most likely to be confused with species with similarly shaped inferior appendages, those with a mesal process that is just preapical and not very strongly projecting, especially C. silausilau, sp. n. and C. vantoli, sp. n. In C. silausilau the apices of the mesal projections of the inferior appendage are more posteriorly directed and the lateral lobe of tergum X more nearly subquadrate in lateral view. In $C$. vantoli the mesal projections of the inferior appendage are almost apical and the laciniate fringe of tergum VIII more elongate. An additional difference is that the mesal lobes of tergum $\mathrm{X}$ in C. noloyan are shorter, flatter, and broader than in related species.

Adult. Color (in alcohol) yellowish-brown. Length of forewing: male $5.0 \mathrm{~mm}$. Forewing venation: stem of Rs curved, slightly sclerotized and thickened at inflection; fork at base of discal cell somewhat thickened, nearly symmetrical, length of discal cell about 2.5 times width; $m$ crossvein proximal to crossveins $s$ and $r-m$, crossvein $s$ not hyaline; $2 \mathrm{~A}$ vein not intersecting 3A (apparently looped to 1A). Postocular 

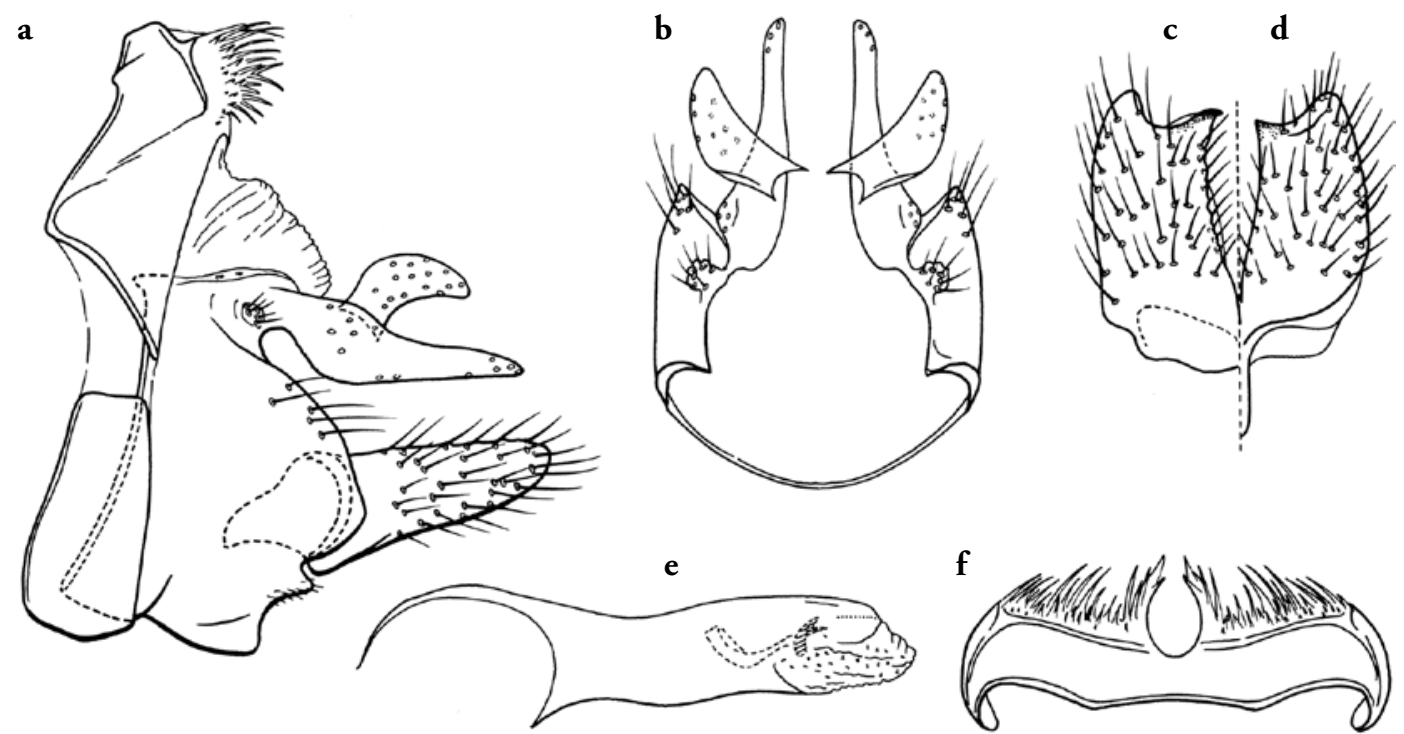

Fig. 24. Chimarra noloyan, male genitalia. - a, lateral; b, segment IX and tergum X, dorsal; c, inferior appendage, ventral; $d$, inferior appendage, dorsal; e, phallic apparatus, lateral; $\mathrm{f}$, tergum VIII, dorsal.

parietal sclerite moderately elongate. Maxillary palps relatively short, segment 3 slightly longer than 2, 2 subequal to 5 . Protarsal claws of male enlarged, asymmetrical in shape and length.

Male genitalia. Abdominal segment VIII short; tergum about as long as sternum, modified, with narrow mesal excavation, excavation boardered laterally with laciniate fringe of variable length; sternum VIII without posteroventral projection. Abdominal segment IX relatively wide dorsolaterally, tergum obsolete dorsomesally, without distinct anterolateral apodemes; anteroventral margin in lateral view not, or scarcely produced; posterior margin distinctly produced; ventral process projecting ventrally, short, broad basally, subtriangular. Preanal appendage setose, very small, rounded. Inferior appendage about as long as tergum X; in lateral view straight, nearly uniformly wide, apex rounded; in ventral view nearly straight, preapically with mesally directed, tooth-like projection. Tergum X with sclerotized lateral lobes and separate mesal lobes, mesal membrane lacking; lateral lobe moderately long, in lateral view narrow, attenuate apically, lobe bearing multiple sensilla; mesal lobe short, thumb-shaped, posterodorsally directed. Phallobase tubular, with pronounced basodorsal expansion, short. Endotheca relatively short, with granularly textured region; without endothecal spines. Phallotremal sclerite complex composed of rod and ring structure, rod short, apically with pair of short, curved, lateral sclerites.

Etymology. This species is named Chimarra noloyan, as a noun in apposition, for the type locality, Sungai ulu Noloyan, where the holotype specimen was collected.

\section{Chimarra phillipsae sp. $\mathbf{n}$.}

Figs 25, 42

Type material. Holotype: $\widehat{\sigma}$, Malaysia, Sabah, Kinabalu National Park, Headquarters, Sg. Liwagu crossing Silau-Silau trail, $06^{\circ} 00^{\prime} \mathrm{N}, 116^{\circ} 33^{\prime} \mathrm{E}, 1470 \mathrm{~m}$, 15.xi.1986, J. Huisman (UMSP000107136) (RMNH).

Paratypes. Malaysia, Sabah, Kinabalu National Park, Headquarters area, 1560 m, 7.ix.1983, Hevel

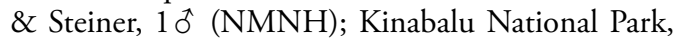
Sungai Liwagu, 1500 m, 10.viii.2005, W. Mey, $1 \delta^{\star}$ (UMHU); Crocker Range, $40 \mathrm{~km} \mathrm{~S}$ of Kota Kinabalu, Sinsuron Rd., 1500 m, 19.xii.1989, J. Huisman, 1 đิ, 1 ( (UMSP); same locality, 20.xii.1989, J. Huisman, $1 \hat{\sigma}^{\hat{\sigma}}$ (UMSP); Poring Hot Spring, 12 km NNE Ranau, Sg. Kepungit, E park boundary, $06^{\circ} 03^{\prime} \mathrm{N}, 116^{\circ} 42^{\prime} \mathrm{E}, 480 \mathrm{~m}, 26 . x i .1986$, J. Huisman, $40^{\circ}$ (UMSP); Poring Hot Spring, $12 \mathrm{~km} \mathrm{NNE}$ Ranau, Sg. Kepungit waterfall, $06^{\circ} 03^{\prime} \mathrm{N}, 116^{\circ} 42^{\prime} \mathrm{E}$, 550, 4.xii.1986, J. Huisman, $20^{\star}$ (RMNH); Kg. Kiau, Sg. Kadamaian, $06^{\circ} 02^{\prime} \mathrm{N}, 116^{\circ} 31^{\prime} \mathrm{E}, 720 \mathrm{~m}$, 26.viii.1986, J. Huisman, $10^{\star}$ (RMNH); $9.5 \mathrm{~km}$ NNW Kundassang, Marei-Parei, Sg. Tawubang, 060' $\mathrm{N}, 116^{\circ} 30^{\prime} \mathrm{E}, 1030 \mathrm{~m}, 11$. iii.1987, J. Huisman, $14 \hat{0}, 1$ 은 

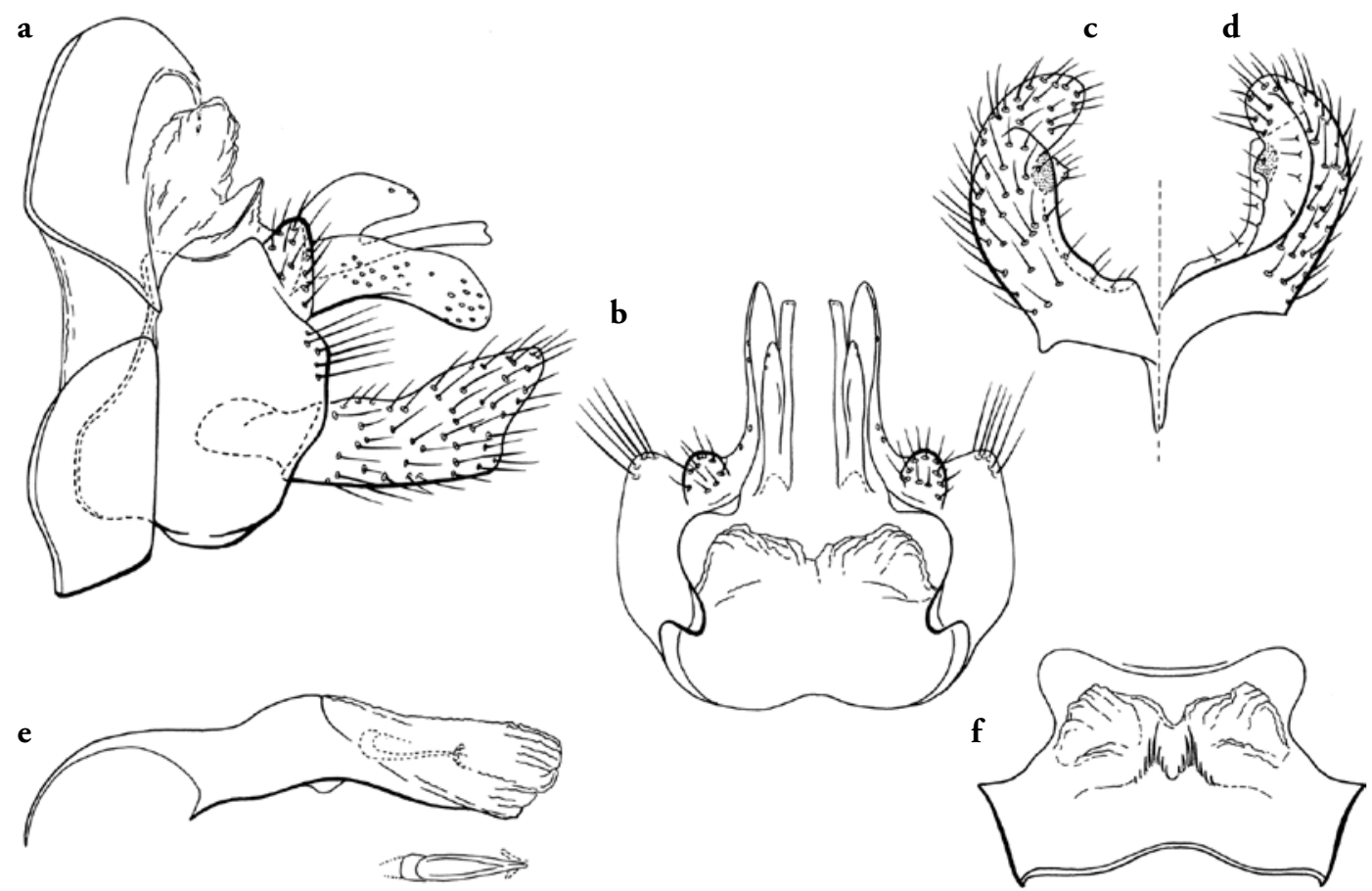

Fig. 25. Chimarra phillipsae, male genitalia. - a, lateral; b, segment IX and tergum X, dorsal; c, inferior appendage, ventral; $\mathrm{d}$, inferior appendage, dorsal; e, phallic apparatus, lateral; inset, phallotremal sclerite, dorsal; f, tergum VIII, dorsal.

Chimarra phillipsae is related to those species of the Chimarra tsudai group with a modified, mesally invaginated, spinose tergum VIII, although the mesal invagination and apical spines in this species are not as pronounced as some other members of this group. Chimarra phillipsae is most readily diagnosed by the distinctive shape of its inferior appendage, which has an apicoventral lobe, making the apex of the appendage appear broadly truncate in lateral view. Another diagnostic feature is the distinctive shape of the mesal lobe of tergum X, which has a rounded basal enlargement and an extending, digitate apical projection.

Adult. Color (in alcohol) brown. Length of forewing: male 5.4-6.2 mm, female 6.8-7.5 mm. Forewing venation: stem of Rs curved; fork at base of discal cell not thickened, nearly symmetrical, length of discal cell about 3 times width; $m$ crossvein somewhat proximal to crossveins $s$ and $r-m$, crossvein $s$ hyaline, in part; $2 \mathrm{~A}$ vein not intersecting 3A (apparently looped to 1A). Postocular parietal sclerite elongate. Maxillary palps moderately elongate, segment 3 subequal to 2, 5 slightly greater than 3, narrow. Protarsal claws of male enlarged, asymmetrical in shape and length.

Male genitalia. Abdominal segment VIII short; tergum about as long as sternum, modified, with small mesal excavation, excavation with short, paired, downturned laciniate mesal processes; sternum VIII without posteroventral projection. Abdominal segment IX relatively wide dorsolaterally, without distinct anterolateral apodemes; anteroventral margin in lateral view slightly produced; posterior margin slightly produced; ventral process projecting ventrally, very short, broad basally, broadly rounded. Preanal appendage setose, short, broadly rounded, broadest basally. Inferior appendage slightly longer than tergum X; in lateral view straight, widest preapically, apex slightly angulate, slightly upturned; in ventral view mesally curved, mesal surface with tooth-like projection at midlength. Tergum $\mathrm{X}$ with sclerotized lateral lobes and separate mesal lobes, mesal membrane lacking; lateral lobe moderately long, in lateral view simple, rounded apically, slightly downcurved, lobe bearing multiple sensilla; mesal lobe with rounded sensillate basal projection and elongate, narrow ventral projection. Phallobase tubular, with pronounced basodorsal expansion, short, ventral apex lightly sclerotized, projecting. Endotheca about as long as phallobase; without endothecal spines. Phallotremal sclerite complex composed of rod and ring 

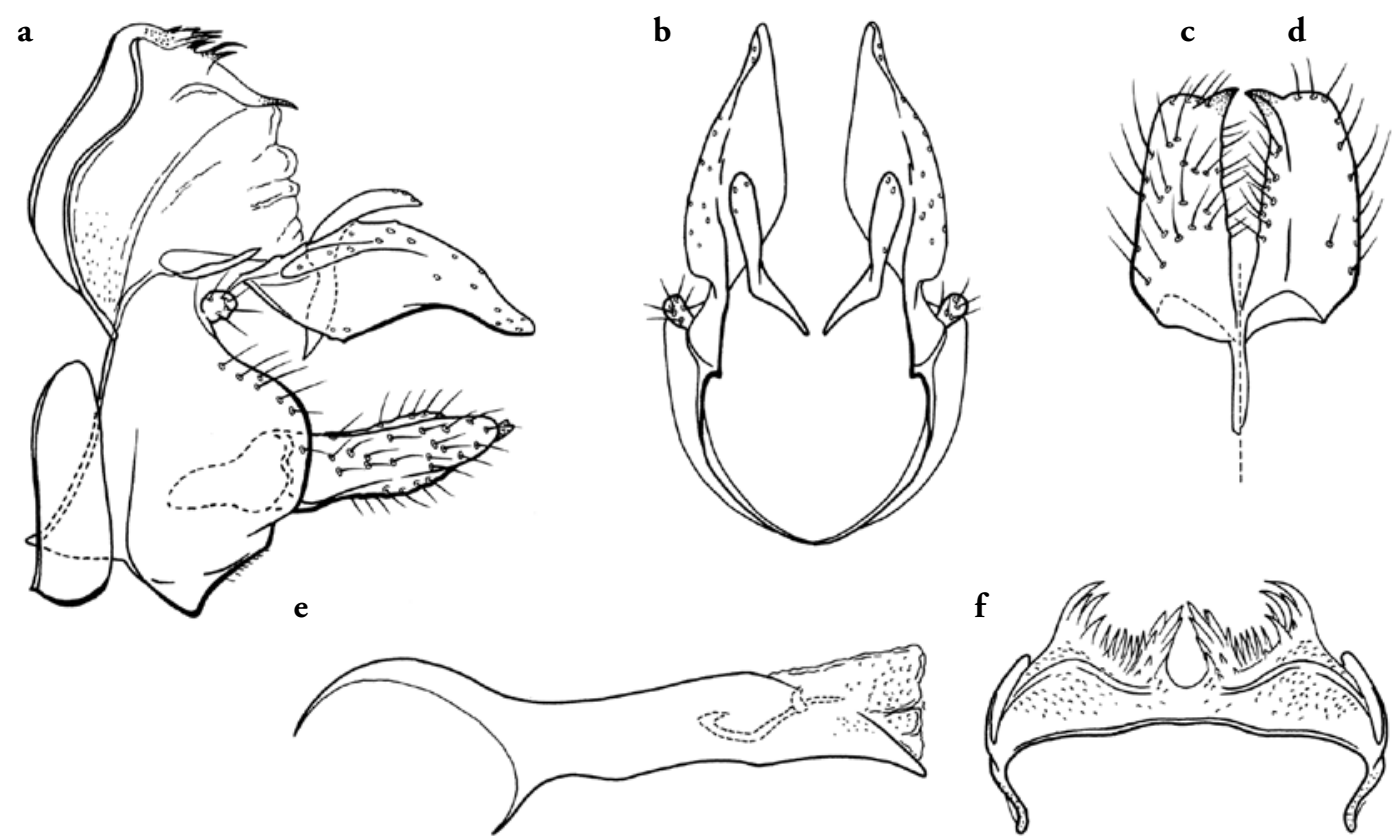

Fig. 26. Chimarra physanoton, male genitalia. - a, lateral; b, segment IX and tergum X, dorsal; c, inferior appendage, ventral; $d$, inferior appendage, dorsal; e, phallic apparatus, lateral; f, tergum VIII, dorsal.

structure, rod moderately elongate, apically with pair of short, curved, lateral sclerites.

Etymology. This species is also named for Anthea Phillips, friend of J. Huisman.

\section{Chimarra physanoton sp. $\mathbf{n}$.}

Fig. 26

Type material. Holotype: $\widehat{\delta}$, Malaysia, Sabah, Kundassang kampong, Sg. Liwagu, on bridge, $06^{\circ} 00^{\prime} \mathrm{N}$, 116 34’ $\mathrm{E}, 1185 \mathrm{~m}$, 23.xi.1986, J. Huisman (UMSP000107246) (RMNH).

Paratypes. Malaysia, Sabah, same locality as holotype, 23.viii.1986, J. Huisman, 1 đ (RMNH); SW Sabah, nr. Long Pa Sia (west), 1050 m, 25.xi-8.xii.1987, Achterberg, 20,10 (UMSP); Long Pa Sia airstrip, $04^{\circ} 24^{\prime} \mathrm{N}, 115^{\circ} 43^{\prime} \mathrm{E}, 1000 \mathrm{~m}, 2-3 . x i i .1989$, J. Huisman, 1 ô (UMSP); Crocker Range, $5 \mathrm{~km} N$ Tenom, base of Sg. ulu Noloyan, $05^{\circ} 10^{\prime} \mathrm{N}, 115^{\circ} 56^{\prime} \mathrm{E}$, 1010 m, 10-11.x.1986, J. Huisman, 1 ô (RMNH); road, Long Pa Sia - Long Semado, Sg. Malabit, $06^{\circ} 21^{\prime} \mathrm{N}, 115^{\circ} 41^{\prime} \mathrm{E}, 1175 \mathrm{~m}, 3-4 . x i i .1987$, J. Huisman, $10^{\tau,} 2$ 우 (UMSP).

Chimarra physanoton is another species belonging to the distinctive subgroup of the Chimarra tsudai group in which tergum VIII is highly modified, invaginated mesally and variously armed with spine-like projections. Generalized features it possesses are a phallotremal sclerite that is not greatly enlarged, and an endotheca that is relatively short and lacks distinctive spines. It is most similar to a group of species from Sabah, including $C$. noloyan, sp. n., C. preapicalis, sp. n., C. silausilau, sp. n., and C. vantoli, sp. n., all of which have similarly shaped inferior appendages, which are short, linear, with an acute mesal projection located preapically, and a tergum VIII that is relatively narrow. It is probably most similar to $C$. silausilau and C. vantoli, differing distinctively from either in the shape of the lateral lobes of tergum X, which are inflated basally, with a lateral crease, and with apices that are downturned and acutely narrowed. Like $C$ silausilau, it has the apicomesal projections of the inferior appendage distinctly posteriorly directed.

Adult. Color (in alcohol) brown. Length of forewing: male 5.7-6.0 mm, female 5.7-6.2 $\mathrm{mm}$. Forewing venation: stem of Rs curved, slightly sclerotized and thickened at inflection; fork at base of discal cell somewhat thickened, nearly symmetrical; $m$ crossvein proximal to crossveins $s$ and $r-m$, crossvein $s$ not hyaline; $2 \mathrm{~A}$ vein not intersecting $3 \mathrm{~A}$ (apparently looped to $1 \mathrm{~A})$. Postocular parietal sclerite short. Maxillary palps relatively short, segment 3 slightly longer than 2, 3 subequal to 5. Protarsal claws of male enlarged, 
a
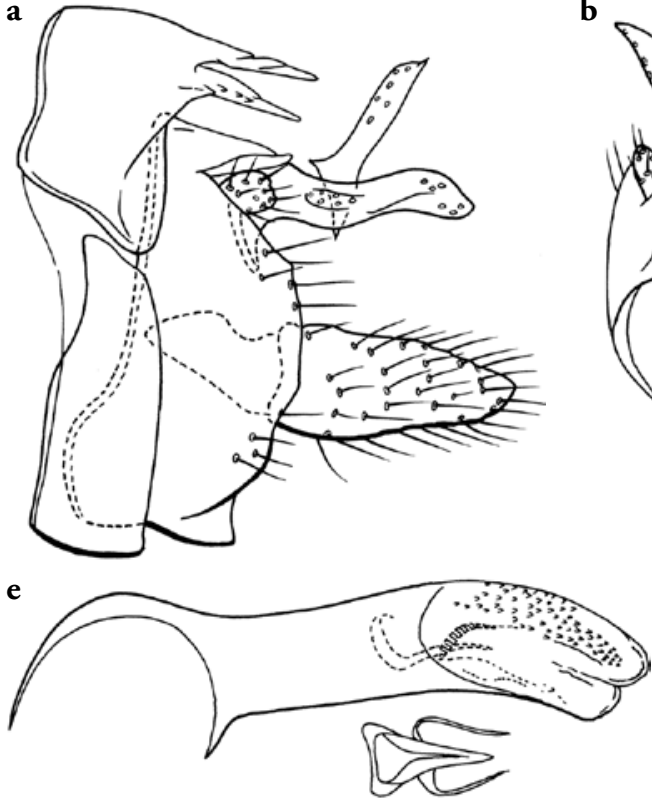

b

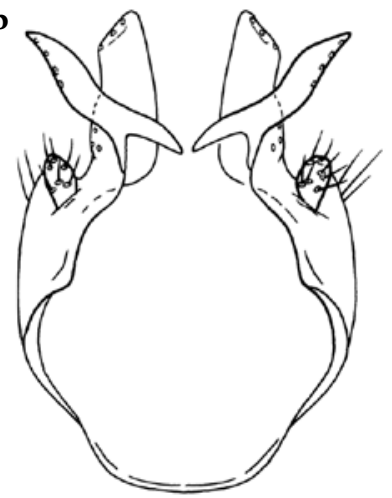

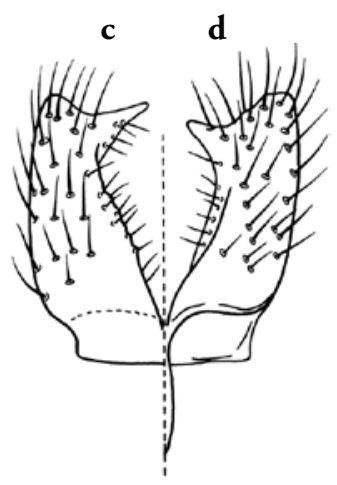

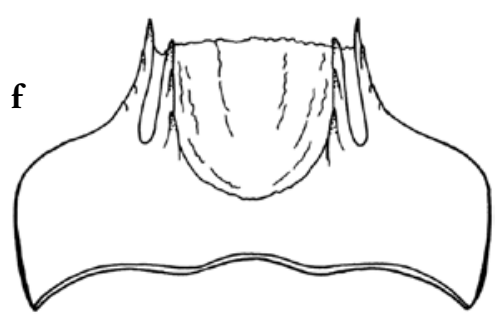

Fig. 27. Chimarra preapicalis, male genitalia. - a, lateral; b, segment IX and tergum X, dorsal; c, inferior appendage, ventral; $d$, inferior appendage, dorsal; e, phallic apparatus, lateral; inset, phallotremal sclerite, dorsal; $f$, tergum VIII, dorsal.

asymmetrical in shape, scarcely different in length.

Male genitalia. Abdominal segment VIII short; tergum about as long as sternum, modified, with small mesal excavation, excavation bordered laterally with laciniate fringe, mesal and lateral fringe more elongate; sternum VIII without posteroventral projection. Abdominal segment IX short dorsolaterally, tergum obsolete dorsomesally; anteroventral margin in lateral view slightly produced; posterior margin sinuously produced; ventral process projecting ventrally, short, broad basally, subtriangular. Preanal appendage setose, very small, rounded. Inferior appendage about as long as tergum $\mathrm{X}$; in lateral view straight, nearly uniformly wide, apex rounded; in ventral view nearly straight, apically with small, posteromesally directed, tooth-like projection. Tergum $\mathrm{X}$ with sclerotized lateral lobes and separate mesal lobes, mesal membrane lacking; lateral lobe long, in lateral view inflated at midlength, apex attenuate, subacute, lobe bearing multiple sensilla; mesal lobe elongate, digitate, slightly posterodorsally curved. Phallobase tubular, with pronounced basodorsal expansion, relatively short, ventral apex distinctly sclerotized, projecting, subacute. Endotheca relatively short, with granularly textured region, without endothecal spines. Phallotremal sclerite complex composed of rod and ring structure, rod moderately elongate, apically with pair of short, curved, lateral sclerites. Etymology. This species is named Chimarra physanoton, used as a noun in apposition, from the Greek words physa, a bag or bellows, and noton, a back, and referring to the basally inflated tergum $\mathrm{X}$ in this species.

\section{Chimarra preapicalis sp. $\mathbf{n}$.}

Fig. 27

Type material. Holotype: o, Malaysia, Sabah, Crocker Range, $40 \mathrm{~km} \mathrm{~S}$ of Kota Kinabalu, Sinsuron Rd., 1500 m, 20.xii.1989, J. Huisman (UMSP000107216) (RMNH).

Paratypes. Malaysia, Sabah, Kinabalu National Park, Sungai Liwagu, 1500 m, 10.viii.2005, W. Mey, $3{ }^{\text {ơ }}$ (UMHU); Kundassang kampong, Sg. Liwagu, on bridge, $06^{\circ} 00^{\prime} \mathrm{N}, 116^{\circ} 34^{\prime} \mathrm{E}, 1185 \mathrm{~m}$, 23.xi.1986, J. Huisman, 1 oิ (RMNH); Kinabalu National Park, Headquarters, Sg. Liwagu, $06^{\circ} 01^{\prime} \mathrm{N}, 116^{\circ} 32^{\prime} \mathrm{E}$, $1500 \mathrm{~m}, 11$. ii.1987, J. Huisman, 1 o (UMSP); $1 \mathrm{~km}$ S Kundasang, 1530 m, 22.viii.1983, Hevel \& Steiner, 10 (NMNH).

Chimarra preapicalis also belongs the distinctive subgroup of the Chimarra tsudai group in which 
tergum VIII is highly modified, invaginated mesally and variously armed with spine-like projections. Generalized features it possesses are a phallotremal sclerite that is not greatly enlarged, and an endotheca that is relatively short and lacks distinctive spines, although the endotheca is "textured" with minute spines. It is most similar to a group of species from Sabah,including C.noloyan,sp.n., C.physanoton,sp.n., C. silausilau, sp. n., and C. vantoli, sp. n., all of which have similarly shaped inferior appendages, which are short, linear, with the acute projections located apicomesally and a tergum VIII that is narrow and tends to have the laciniate fringe formed into 2 pairs of projections, margined with spines of variable length. From other species in this group it is most diagnostically distinguished by details in shape of the inferior appendages, which have the acute apicomesal projections emerging more preapically (farther from the apex) than most members of this group and are also more prominently developed. Details in the shape of the lateral lobes of tergum $\mathrm{X}$ and development of the armature of tergum VIII are also useful adjunct characters.

Adult. Color (in alcohol) light brown. Length of forewing: male $4.8-5.6 \mathrm{~mm}$. Forewing venation: stem of Rs curved, slightly sclerotized and thickened at inflection; fork at base of discal cell somewhat thickened, nearly symmetrical, length of discal cell about 3 times width; $m$ crossvein proximal to crossveins $s$ and $r-m$, crossvein $s$ not hyaline; $2 \mathrm{~A}$ vein not intersecting 3A (apparently looped to 1A). Postocular parietal sclerite short. Maxillary palps moderately elongate, segment 3 slightly greater than 2 , 3 subequal to 5 . Protarsal claws of male enlarged, asymmetrical in shape and length.

Male genitalia. Abdominal segment VIII short; tergum longer than sternum, modified, with mesal excavation, excavation apicolaterally with bifurcating, laciniate processes; sternum VIII without posteroventral projection. Abdominal segment IX relatively wide dorsolaterally, tergum obsolete dorsomesally, without distinct anterolateral apodemes; anteroventral margin in lateral view slightly produced, rounded; posterior margin distinctly produced; ventral process projecting ventrally, broad basally, subtriangular. Preanal appendage setose, small, rounded, slightly narrowed basally. Inferior appendage slightly longer than tergum $X$; in lateral view straight, widest basally, gradually narrowing distally, apex rounded; in ventral view nearly straight, preapically with prominent, mesal, tooth-like projection. Tergum X with sclerotized lateral lobes and separate mesal lobes, mesal membrane lacking; lateral lobe moderately long, in lateral view narrow, apex slightly downcurved, lobe bearing multiple sensilla; mesal lobe elongate, digitate, posterolaterally directed, apex acute. Phallobase tubular, with pronounced basodorsal expansion, short. Endotheca relatively short, with granularly textured region; without endothecal spines. Phallotremal sclerite complex composed of rod and ring structure, rod moderately elongate, preapically with short lateral sclerites.

Etymology. This species is named Chimarra preapicalis because the mesal projections of the inferior appendages are located more preapically (farther from the apex) than in closely related species.

\section{Chimarra prokrustes Malicky}

Fig. 28

Chimarra prokrustes Malicky, 2008: 840, holotype ô, Indonesia (Eastern Kalimantan), Zoologische Museum Lausanne.

Material examined. Malaysia, Sabah, $18 \mathrm{~km}$ on road Keningau-Kimanis, gravel quarry, $05^{\circ} 26^{\prime} \mathrm{N}, 116^{\circ} 05^{\prime} \mathrm{E}$, 1050 m, 20.i.1987, J. Huisman, 1 ơ (RMNH); $12 \mathrm{~km}$ NNE Ranau, Poring Hot Spring, Sg. Tananansad, $06^{\circ} 03^{\prime} \mathrm{N}$, $16^{\circ} 42^{\prime} \mathrm{E}, 560 \mathrm{~m}$, 28.viii.1986, J. Huisman, 1 ơ $^{\text {(UMSP); }}$ $12 \mathrm{~km}$ NNE Ranau, Poring Hot Spring, Sg. Kepungit, $06^{\circ} 03^{\prime} \mathrm{N}, 116^{\circ} 42^{\prime} \mathrm{E}, 550 \mathrm{~m}, 24.1 .1987$, J. Huisman, $1 \widehat{o}^{\circ}$ (UMSP).

Chimarra prokrustes resembles those species from Borneo in the Chimarra tsudai group that have an unmodified, but somewhat widened tergum VIII, an acute projection or tooth on the dorsal margin of the inferior appendages, evident in lateral view, and mesal lobes of tergum $X$ that are sclerotized, upturned and have an acute apex. Species in this group include C. denticula, sp. n., C. karlijnae, sp. n., C. scolops, sp. n., C. sinitorum, sp. n., and C. stenodactylus, sp. n. All of these species also have a phallic apparatus with numerous small spines and a narrow sclerous dorsal extension, extending over the basal part of the endotheca. As previously discussed, these species are all easily distinguished from one another by the characteristic and species-specific shapes of the lateral and mesal lobes of tergum X, as well as by differences in the shape of the inferior appendages. Among these species, Chimarra prokrustes is easily diagnosed by the very elongate and typically undulate lateral lobes of tergum X, and by the widened base of the dorsomesal process of the inferior appendage. As compared to the illustration of the holotype, the form figured here seems to differ in that the lateral lobes of tergum $\mathrm{X}$ are more irregular in shape also have distinct, sensillate basolateral protrusions, as well as inferior appendages, as viewed ventrally, with their apices less narrowed. Compared 

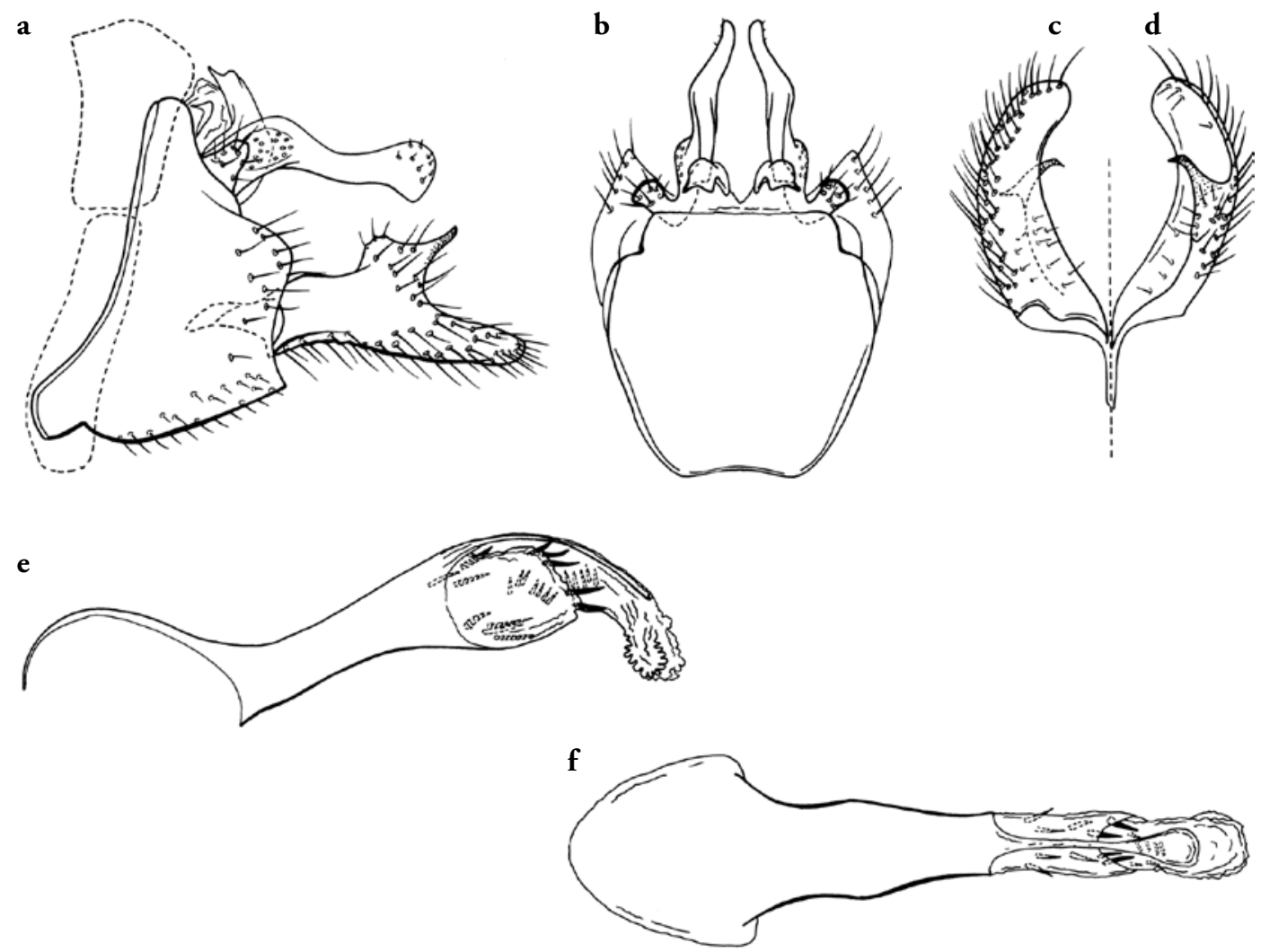

Fig. 28. Chimarra prokrustes, male genitalia. - a, lateral; b, segment IX and tergum X, dorsal; c, inferior appendage, ventral; $\mathrm{d}$, inferior appendage, dorsal; e, phallic apparatus, lateral; $\mathrm{f}$, phallic apparatus, dorsal.

to the differences that separate this species from its most closely related congeners, these discrepancies are minor and probably not significant.

Adult. Color (in alcohol) yellowish-brown. Length of forewing: 4.4-5.0 mm. Forewing venation: stem of Rs curved, slightly sclerotized and thickened at inflection; fork at base of discal cell not thickened, nearly symmetrical, length of discal cell about 3 times width; $m$ crossvein proximal to crossveins $s$ and $r-m$, crossvein $s$ hyaline, in part; $2 \mathrm{~A}$ vein not intersecting 3A (apparently looped to 1A). Postocular parietal sclerite short. Maxillary palps relatively short, segment 3 slightly longer than 2, 3 subequal to 5. Protarsal claws of male enlarged, asymmetrical in shape and length.

Male genitalia. Abdominal segment VIII short; tergum longer than sternum, unmodified; sternum VIII without posteroventral projection. Abdominal segment IX short dorsolaterally, tergum obsolete dorsomesally, without distinct anterolateral apodemes; anteroventral margin in lateral view produced, ventral margin rounded mesally as viewed dorsoventrally; posterior margin distinctly produced; ventral process absent or nearly so. Preanal appendage setose, short, rounded, broadest basally. Inferior appendage longer than tergum X; in lateral view straight, widest at midlength, narrow distally, apex rounded, dorsal margin with very basally wide, abruptly apically acute projection at midlength; in ventral view somewhat mesally curved, mesal surface without processes. Tergum X with sclerotized lateral lobes and separate mesal lobes, mesal membrane lacking; lateral lobe long, in lateral view narrow, undulate, rounded apically, lobe bearing multiple sensilla; mesal lobe short, digitate, distinctly sclerotized, dorsally directed, in lateral view, narrow, recurved, apex with single, acuminate spine-like projection. Phallobase tubular, with pronounced basodorsal expansion, moderately elongate, relatively narrow, dorsal margin extended apically, elongate, narrow, lightly sclerotized. Endotheca length not discernable (not expanded); with numerous short endothecal spines. Phallotremal sclerite complex not evident in material examined. 

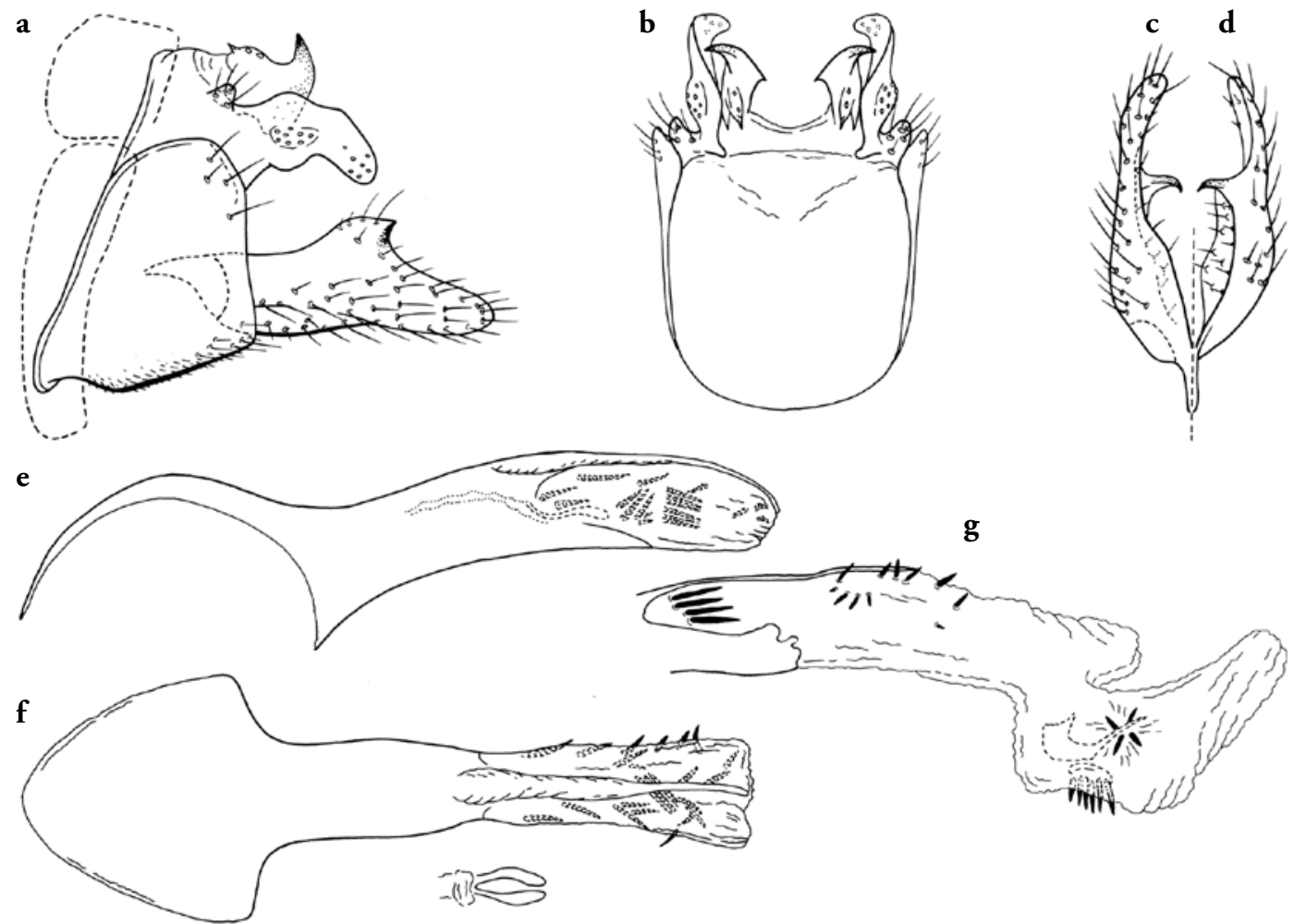

Fig. 29. Chimarra scolops, male genitalia. - a, lateral; b, segment IX and tergum $X$, dorsal; $c$, inferior appendage, ventral; $\mathrm{d}$, inferior appendage, dorsal; e, phallic apparatus, lateral; $\mathrm{f}$, phallic apparatus, dorsal; inset phallotremal sclerite, ventral; g, expanded endotheca, lateral.

\section{Chimarra scolops sp. $\mathbf{n}$.}

Fig. 29

Type material. Holotype: $\delta$, Malaysia, Sabah, Kinabalu National Park, Headquarters, Sg. Liwagu crossing Silau-Silau trail, $06^{\circ} 00^{\prime} \mathrm{N}, 116^{\circ} 33^{\prime} \mathrm{E}, 1470 \mathrm{~m}$, 15.viii.1986, J. Huisman (UMSP000208645) (RMNH).

Paratypes. Malaysia, Sabah, same data as holotype, 20 (UMSP); Kinabalu National Park, headquarters, Sungai Silau-Silau, 11.viii.2005, W. Mey, 30, 1 우 (UMHU); Kinabalu National Park, Sungai Liwagu, 1100 m, 12.viii.2005, W. Mey, 4 đ (UMHU); same locality, 15.xi.1986, J. Huisman, 1 के (RMNH); Crocker Range, $40 \mathrm{~km} \mathrm{~S}$ of Kota Kinabalu, Sinsuron Rd., 1500 m, 18.xii.1989, J. Huisman, 20 (UMSP), same locality, 20.xii.1989, J. Huisman, 1 ऊิ (RMNH).

Chimarra scolops resembles those species in the Chimarra tsudai group from Sabah that have an unmodified, but somewhat widened tergum VIII, an acute projection or tooth on the dorsal margin of the inferior appendages, evident in lateral view, and mesal lobes of tergum X that are sclerotized, upturned and have an acute apex. Species in this group include C. denticula, sp. n., C. karlijnae, sp. n., C. prokrustes Malicky, C. sinitorum, sp. n., and C. stenodactylus, sp. $\mathrm{n}$. All of these species also have a phallic apparatus with numerous small spines and a narrow sclerous dorsal extension, extending over the basal part of the endotheca. These species are all easily distinguished from one another by the characteristic and speciesspecific shapes of the lateral and mesal lobes of tergum X, as well as by differences in the shape of the inferior appendages. Among these species, Chimarra scolops is probably most similar to $C$. denticulata, but differs in both the shape of the lateral lobes of tergum X and in lacking a preapical "denticle" or toothlike projection on the inferior appendage.

Adult. Color (in alcohol) yellowish-brown. Length of forewing: male $5.8-6.5 \mathrm{~mm}$. Forewing venation: stem of Rs curved, distinctly sclerotized and thickened at inflection; fork at base of discal cell distinctly thickened, nearly symmetrical, length of discal cell about 2.5 times width; $m$ crossvein proximal to 

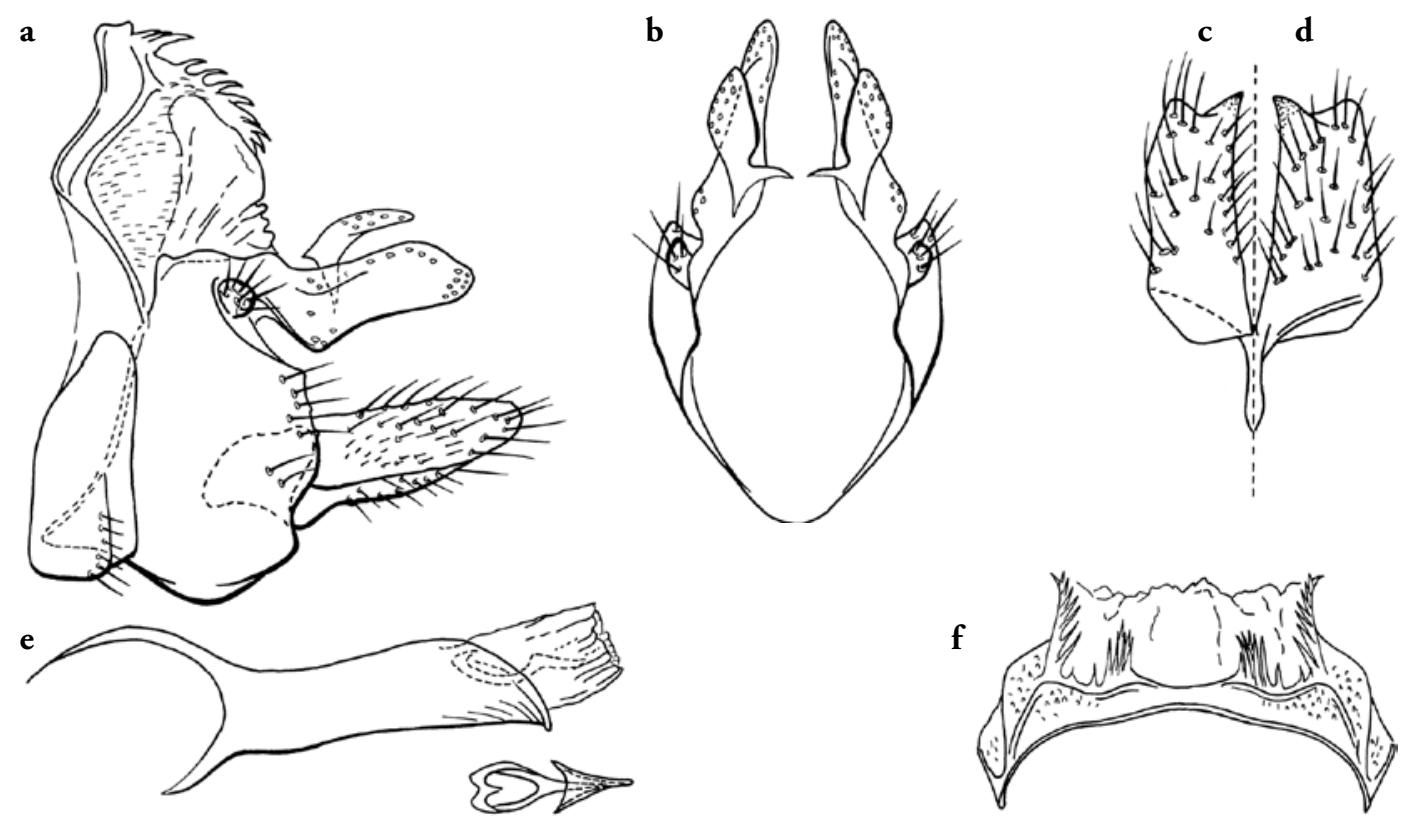

Fig. 30. Chimarra silausilau, male genitalia. - a, lateral; b, segment IX and tergum $X$, dorsal; $c$, inferior appendage, ventral; $d$, inferior appendage, dorsal; e, phallic apparatus, lateral; inset, phallotremal sclerite, dorsal; f, tergum VIII, dorsal.

crossveins $s$ and $r$ - $m$, crossvein $s$ hyaline; $2 \mathrm{~A}$ vein not intersecting 3A (apparently looped to 1A). Postocular parietal sclerite moderately elongate, head very large. Maxillary palps elongate, segment 3 subequal to 2 and 5. Protarsal claws of male enlarged, asymmetrical in shape and length.

Male genitalia. Abdominal segment VIII moderate in length; tergum distinctly longer than sternum, unmodified; sternum VIII without posteroventral projection. Abdominal segment IX relatively wide dorsolaterally, tergum obsolete dorsomesally, without distinct anterolateral apodemes; anteroventral margin in lateral view not, or scarcely produced; posterior margin slightly produced; ventral process absent or nearly so. Preanal appendage setose, short, rounded, broadest basally. Inferior appendage much longer than tergum $\mathrm{X}$; in lateral view straight, nearly uniformly wide, apex broadly rounded, dorsal margin with very basally wide, apically acute projection at midlength; in ventral view very slightly mesally curved, nearly straight, mesal surface without processes. Tergum X with sclerotized lateral lobes and separate mesal lobes, mesal membrane lacking; lateral lobe short, in lateral view irregular, rounded apically, distinctly downcurved, lobe bearing multiple sensilla; mesal lobe short, digitate, distinctly sclerotized, dorsally directed, with basal sensillate lobe and strongly dorsally hooked apical projection.
Phallobase tubular, with pronounced basodorsal expansion, moderately elongate, ventral apex lightly sclerotized, slightly projecting, dorsal margin extended apically, elongate, narrow, lightly sclerotized. Endotheca elongate, with several tracts of short spines, 1 basally, 1 dorsally in basal half, and 1 apically or preapically, composed of ventral group of spines and paired lateral groups of several spines. Phallotremal sclerite complex composed of rod and ring structure, rod relatively short.

Etymology. This species is named Chimarra scolops, a noun in apposition, from the Greek word skolops, a thorn, and referring to the thorn-like apices of the mesal processes of tergum $\mathrm{X}$ in this species.

\section{Chimarra silausilau sp. $\mathbf{n}$.}

Fig. 30

Type material. Holotype: $\widehat{\sigma}$, Malaysia, Sabah, Kinabalu National Park, Sg. Silau-Silau, $06^{\circ} 00^{\prime} \mathrm{N}$, $116^{\circ} 32^{\prime} 12^{\prime \prime E}, 1560 \mathrm{~m}$, bridge near Bukit Burung trail, 20-21.xi.1986, J. Huisman(UMSP000107237) (RMNH).

Paratypes. Malaysia, Sabah, same data as holotype, 2 đo, 3 ㅇ (UMSP); Kinabalu National Park, Sg. Tibabar @ Liwagu trail, 06 $02^{\prime} \mathrm{N}, 116^{\circ} 33^{\prime} \mathrm{E}, 1750 \mathrm{~m}$, 2.x.1986, J. Huisman, 10, 3 ㅇ (RMNH). 
Chimarra silausilau belongs to the distinctive subgroup of the Chimarra tsudai group in which tergum VIII is highly modified, invaginated mesally and variously armed with spine-like projections. Generalized features it possesses are a phallotremal sclerite that is not greatly enlarged, and an endotheca that is relatively short and lacks distinctive spines, although the endotheca is "textured" with minute spines. It is most similar to a group of species from Sabah, including C. noloyan, sp. n., C. physanoton, sp. n., C. preapicalis, sp. n., and C. vantoli, sp. n., all of which have similarly shaped inferior appendages, which are short, linear, and with the acute projections located apicomesally. All of these species also have a tergum VIII that is particularly narrow. Chimarra silausilau is probably most similar to C. physanoton and C. vantoli, differing from either in having the apex of the lateral lobes of tergum $X$ more nearly subquadrate (less angular apically). Like C. physanoton, it has the apicomesal projections of the inferior appendage posteriorly directed.

Adult. Color (in alcohol) light brown. Length of forewing: male $6.0-6.5 \mathrm{~mm}$, female $6.5-6.8 \mathrm{~mm}$. Forewing venation: stem of Rs curved, slightly sclerotized and thickened at inflection; fork at base of discal cell somewhat thickened, nearly symmetrical, length of discal cell about 3 times width; $m$ crossvein proximal to crossveins $s$ and $r-m$, crossvein $s$ not hyaline; $2 \mathrm{~A}$ vein not intersecting $3 \mathrm{~A}$ (apparently looped to $1 \mathrm{~A}$ ). Postocular parietal sclerite moderately elongate. Maxillary palps moderately elongate, 3 distinctly longer than 2, 5 slightly shorter than 3 . Protarsal claws of male enlarged, asymmetrical in shape and length.

Male genitalia. Abdominal segment VIII short; tergum about as long as sternum, modified, with mesal excavation, excavation apicolaterally with bifurcating, laciniate processes, lateral process more elongate; sternum VIII without posteroventral projection. Abdominal segment IX relatively wide dorsolaterally, tergum obsolete dorsomesally, without distinct anterolateral apodemes; anteroventral margin in lateral view slightly produced; posterior margin distinctly produced; ventral process projecting ventrally, very short, broad basally, broadly rounded. Preanal appendages setose, very small, rounded. Inferior appendage about as long as tergum $\mathrm{X}$; in lateral view straight, nearly uniformly wide, apex rounded; in ventral view nearly straight, apically with posteromesally directed, tooth-like projection. Tergum $\mathrm{X}$ with sclerotized lateral lobes and separate mesal lobes, mesal membrane lacking; lateral lobe moderately long, in lateral view simple, subquadrate, apex rounded, lobe bearing multiple sensilla; mesal lobe short, digitate, posterodorsally directed, apex flattened. Phallobase tubular, with pronounced basodorsal expansion, short, ventral apex weakly sclerotized, slightly projecting. Endotheca relatively short, granularly textured region not discernable; without endothecal spines. Phallotremal sclerite complex composed of rod and ring structure, rod short, apically with pair of short, curved, lateral sclerites.

Etymology. This species is named Chimarra silausilau, as a noun in apposition, for the stream, Sungai SilauSilau, where the holotype specimen was collected.

\section{Chimarra sinitorum sp. $\mathbf{n}$.}

Fig. 31

Type material. Holotype: $\widehat{\delta}$, Malaysia, Sabah, Poring Hot Spring, $12 \mathrm{~km}$ NNE Ranau, Sg. Montokungon, $06^{\circ} 02^{\prime} \mathrm{N}, 116^{\circ} 42^{\prime} \mathrm{E}, 525 \mathrm{~m}, 30 . i .1987$, J. Huisman (UMSP000107298) (RMNH).

Paratypes. Malaysia, Sabah, same data as holotype, 30 , 3 (UMSP); 12 km NNE Ranau, Poring Hot Springs, Sg. Langanan, 980 m, 29.viii.1986, J. Huisman, 20 , $5 \uparrow$ (UMSP); Poring Hot Spring, $12 \mathrm{~km}$ NNE Ranau, confluence Sg. Kepungit \& Sg. Langanan, $06^{\circ} 03^{\prime} \mathrm{N}, 116^{\circ} 43^{\prime} \mathrm{E}, 450 \mathrm{~m}$, 29.i.1987, J. Huisman, $10^{\text {tे }}(\mathrm{RMNH}) ; 12 \mathrm{~km}$ NNE Ranau, Poring Hot Spring, Sg. Kepungit, $06^{\circ} 03^{\prime} \mathrm{N}$, $116^{\circ} 42^{\prime} \mathrm{E}, 480$ m, 27.i.1987, J. Huisman, $10^{\top}$ (RMNH); same locality, 550 m, 24.i.1987, J. Huisman, 20 oิ (UMSP) Poring Hot Spring, $12 \mathrm{~km} \mathrm{NNE}$ Ranau, Sg. Kepungit, E park boundary, $06^{\circ} 03^{\prime} \mathrm{N}$, 116²'', 480 m, 26.xi.1986, J. Huisman, 40, 5 ㅇ (RMNH); Poring Hot Spring, $12 \mathrm{~km} \mathrm{NNE} \mathrm{Ranau,}$ Sg. Kepungit waterfall, $06^{\circ} 03^{\prime} \mathrm{N}, 116^{\circ} 42^{\prime} \mathrm{E}, 550 \mathrm{~m}$, 4.xii.1986, J. Huisman, $20^{\star}$ (UMSP); same locality, 625 m, 29.viii.1986, J. Huisman, 1 ô, 3 ; 12 km NNE Ranau, Poring Hot Spring, Sg. Tananansad, $06^{\circ} 03^{\prime} \mathrm{N}, 116^{\circ} 42^{\prime} \mathrm{E}, 560 \mathrm{~m}$, 28.viii.1986, J. Huisman, 20 (RMNH).

Chimarra sinitorum resembles those species from Borneo in the Chimarra tsudai group that have an unmodified, but somewhat widened tergum VIII, an acute projection or tooth on the dorsal margin of the inferior appendages, evident in lateral view, and mesal lobes of tergum $\mathrm{X}$ that are sclerotized, upturned and have an acute apex. Species in this group include $C$. denticula, sp. n., $C$. karlijnae, sp. n., C. prokrustes Malicky, C. scolops, sp. n., and C. stenodactylus, sp. n. All of these species also have a phallic apparatus with numerous small spines and a narrow sclerous dorsal extension, extending over the basal part of the endotheca. These species are all easily distinguished from one another by the characteristic and species-specific shapes of the lateral and 
a
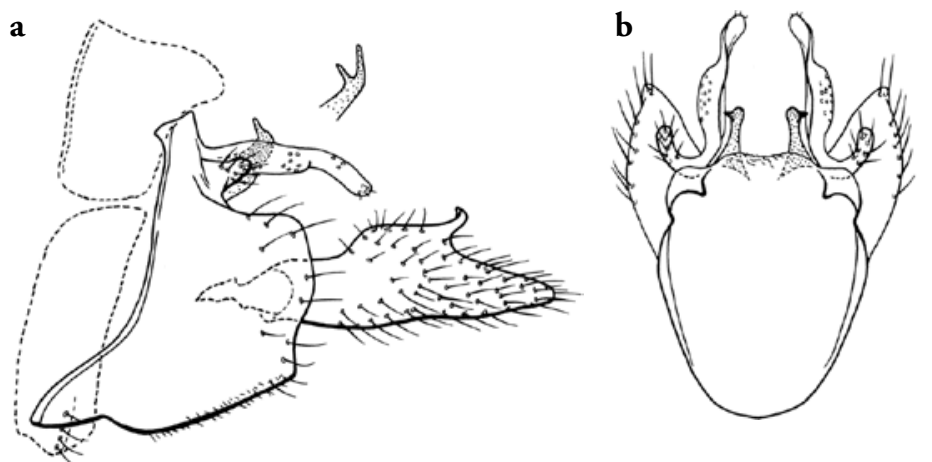
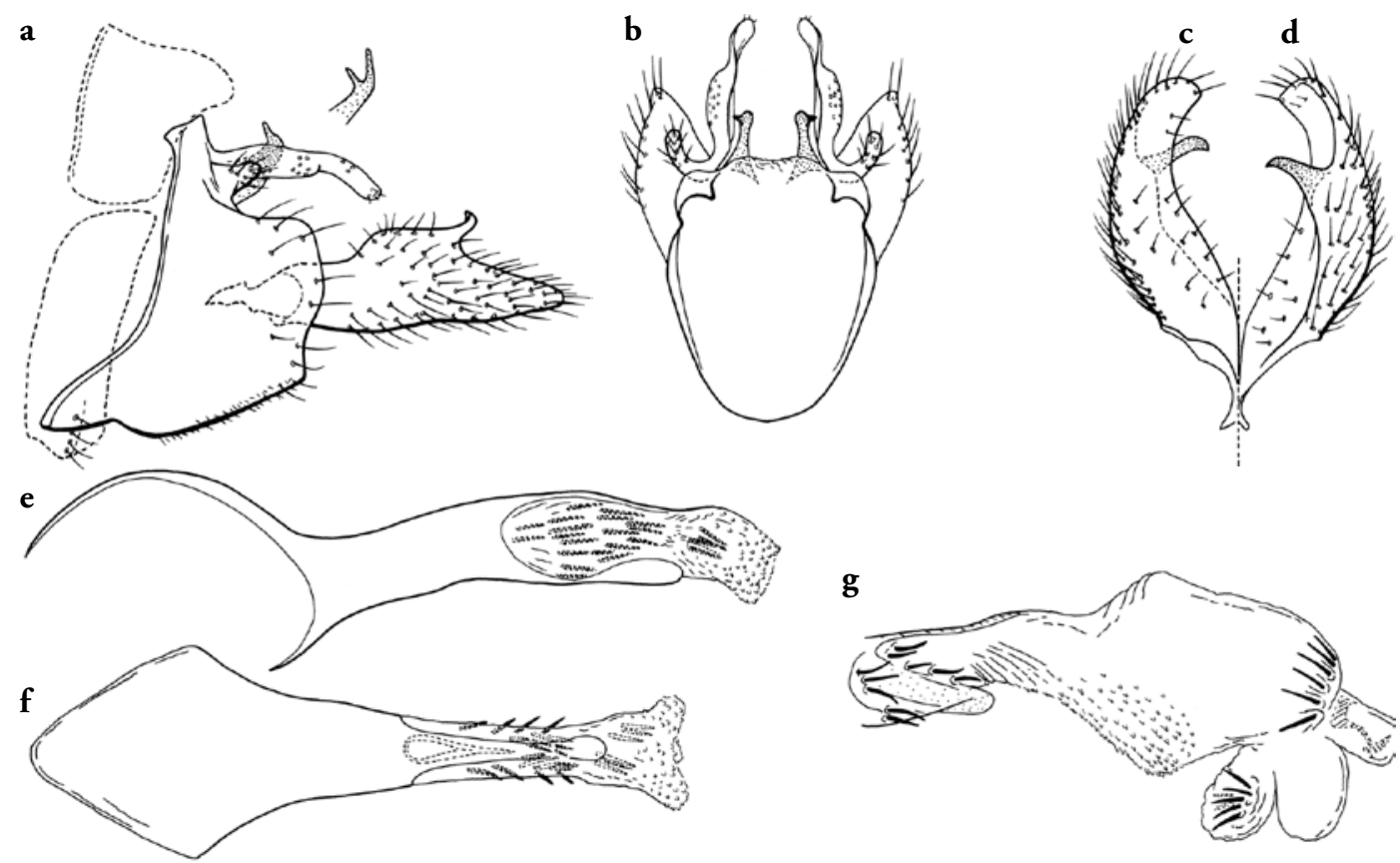

Fig. 31. Chimarra sinitorum, male genitalia. - a, lateral; b, segment IX and tergum X, dorsal; c, inferior appendage, ventral; $d$, inferior appendage, dorsal; e, phallic apparatus, lateral; f, tergum VIII, dorsal.

mesal lobes of tergum $\mathrm{X}$, as well as by differences in the shape of the inferior appendages. Among these species, Chimarra sinitorum is distinctive both for the shape of the inferior appendages, which have a very broad enlargement basal to the acute mesal projection, and also for the narrow lateral lobe of tergum $\mathrm{X}$ and the strongly hooked mesal lobe with one or two acute apical projections, the bidactylate condition apparently most common.

Adult. Color (in alcohol) yellowish-brown. Length of forewing: male $4.5-5.3 \mathrm{~mm}$, female $5.0-5.8 \mathrm{~mm}$. Forewing venation: stem of Rs curved, slightly sclerotized and thickened at inflection; fork at base of discal cell distinctly thickened, nearly symmetrical, length of discal cell about 3 times width; $m$ crossvein proximal to crossveins $s$ and $r-m$, crossvein $s$ hyaline; $2 \mathrm{~A}$ vein not intersecting 3A (apparently looped to 1A). Postocular parietal sclerite short. Maxillary palps moderately elongate, segment 3 slightly greater than 2, 3 subequal to 5 . Protarsal claws of male enlarged, asymmetrical in shape and length.

Male genitalia. Abdominal segment VIII short; tergum longer than sternum, unmodified; sternum VIII without posteroventral projection. Abdominal segment IX short dorsolaterally, tergum obsolete dorsomesally, without distinct anterolateral apodemes; anteroventral margin in lateral view produced, posterior margin distinctly produced; ventral process absent or nearly so. Preanal appendage setose, small, rounded, slightly narrowed basally. Inferior appendage much longer than tergum $\mathrm{X}$; in lateral view straight, widest mesally, gradually narrowing distally, apex rounded, dorsal margin with very basally wide, abruptly apically acute projection at midlength; in ventral view somewhat mesally curved, mesal surface without processes. Tergum X with sclerotized lateral lobes and separate mesal lobes, mesal membrane lacking; lateral lobe short, in lateral view narrow, apex downturned, lobe bearing multiple sensilla; mesal lobe short, digitate, distinctly sclerotized, dorsally directed, in lateral view, narrow, apex with 1 or 2 acuminate projections. Phallobase tubular, with pronounced basodorsal expansion, moderately elongate, relatively narrow, ventral apex lightly sclerotized, projecting, dorsal margin extended apically, elongate, narrow, lightly sclerotized. Endotheca moderately elongate, bulbously inflated, with minutely spinulose ventral region and numerous short endothecal spines in several tracts, 1 basal, 1 apicodorsal, and 1 apicoventral. Phallotremal sclerite complex composed of rod and ring structure, rod relatively short, with short apicolateral sclerites.

Etymology. Named for Gabriel Sinit and his family, in recognition of the friendship and assistance they afforded to J. Huisman during her work and travels on Borneo. 

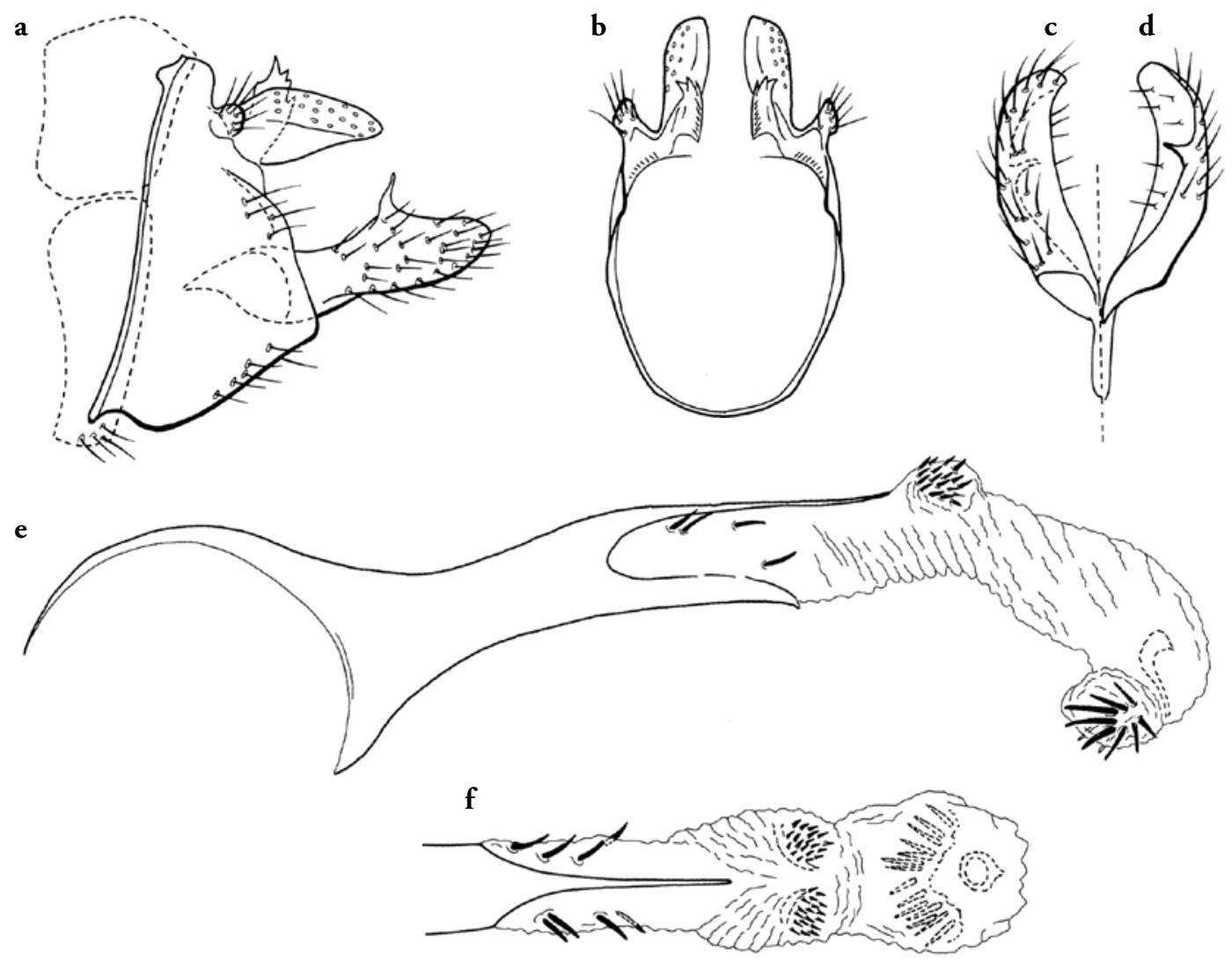

Fig. 32. Chimarra stenodactylus, male genitalia. - a, lateral; b, segment IX and tergum X, dorsal; c, inferior appendage, ventral; $d$, inferior appendage, dorsal; e, phallic apparatus, lateral; $f$, expanded endotheca, dorsal.

\section{Chimarra stenodactylus sp. $\mathbf{n}$.}

Fig. 32

Type material. Holotype: $\widehat{\delta}$, Malaysia, Sabah, $60 \mathrm{~km}$ W Lahad Datu, Danum Valley Field Centre, Sg. Palum Tambun \& vicinity, $04^{\circ} 58^{\prime} \mathrm{N}$, $117^{\circ} 48^{\prime} \mathrm{E}, 150 \mathrm{~m}, 19-26 . i i i .1987$, J. Huisman (UMSP000107286) (RMNH).

Paratypes. Malaysia, Sabah, same data as holotype, 10 (RMNH); Danum Valley Field Centre, nature trail brooklet, $04^{\circ} 58^{\prime} \mathrm{N}, 117^{\circ} 48^{\prime} \mathrm{E}, 180 \mathrm{~m}$, 9.ix.1986, J. Huisman, 20 (UMSP).

Chimarra stenodactylus resembles those species from Borneo in the Chimarra tsudai group that have an unmodified, but somewhat widened tergum VIII, an acute projection or tooth on the dorsal margin of the inferior appendages, evident in lateral view, and mesal lobes of tergum $\mathrm{X}$ that are sclerotized, upturned and have an acute apex. Species in this group include C. denticula, sp. n., C. karlijnae, sp. n.,
C. prokrustes Malicky, C. scolops, sp. n., and C. sinitorum, sp. n. All of these species also have a phallic apparatus with numerous small spines and a narrow sclerous dorsal extension, extending over the basal part of the endotheca. These species are all easily distinguished from one another by the characteristic and species-specific shapes of the lateral and mesal lobes of tergum $\mathrm{X}$, as well as by differences in the shape of the inferior appendages. Among these species, $C$. sinitorum is diagnosed by the shape of the inferior appendages, which have a very narrow base to the acute mesal projection, and also by the very short, simple, rounded lateral lobes of tergum X.

Adult. Color (in alcohol) yellowish-brown. Length of forewing: male $4.5-4.9 \mathrm{~mm}$. Forewing venation: stem of Rs curved, slightly sclerotized and thickened at inflection; fork at base of discal cell distinctly thickened, nearly symmetrical, length of discal cell about 2 times width; $m$ crossvein proximal to crossveins $s$ and $r-m$, crossvein $s$ hyaline; $2 \mathrm{~A}$ vein not intersecting 3A (apparently looped to 1A). Postocular parietal 
sclerite short. Maxillary palps moderately elongate, segment 3 subequal to 2 and 5. Protarsal claws of male enlarged, asymmetrical in shape and length.

Male genitalia. Abdominal segment VIII moderate in length; tergum distinctly longer than sternum, modified, with weakly developed V-shaped mesal depression; sternum VIII without posteroventral projection. Abdominal segment IX relatively wide dorsolaterally, tergum obsolete dorsomesally, without distinct anterolateral apodemes; anteroventral margin in lateral view slightly produced, ventral margin rounded mesally as viewed dorsoventrally; posterior margin distinctly produced; ventral process absent or nearly so. Preanal appendage setose, small, rounded, slightly narrowed basally. Inferior appendage longer than tergum $\mathrm{X}$; in lateral view straight, nearly uniformly wide, apex broadly rounded, dorsal margin with basally narrow, acute projection at midlength; in ventral view somewhat mesally curved, mesal surface without processes. Tergum $X$ with sclerotized lateral lobes and separate mesal lobes, mesal membrane lacking; lateral lobe short, in lateral view simple, rounded apically, lobe bearing multiple sensilla; mesal lobe short, digitate, distinctly sclerotized, dorsally directed, apex with multiple, spine-like projections. Phallobase tubular, with pronounced basodorsal expansion, moderately elongate, relatively narrow, ventral apex lightly sclerotized, projecting, dorsal margin extended apically, elongate, narrow, lightly sclerotized. Endotheca elongate, tubular; with 3 tracks of small endothecal spines, basal track of few scattered spines, paired, dorsal track of very short spines at midlength, apical track of slightly longer spines. Phallotremal sclerite complex composed of rod and ring structure, rod very short.

Etymology. This species is named C. stenodactylus from the Greek words stenos, narrow, and dactylos, a finger, used as a noun in apposition, and referring to the dorsal projection on the inferior appendage, which is very narrow basally compared to other related species.

\section{Chimarra thaumas Malicky}

Figs 33, 43

Chimarra thaumas Malicky, 2008: 840, holotype $\delta$, Indonesia (Eastern Kalimantan), Zoologische Museum Lausanne.

Material examined. Malaysia, Sabah, Crocker Range, $5 \mathrm{~km} \mathrm{~N}$ Tenom, base of Sg. ulu Noloyan, $05^{\circ} 10^{\prime} \mathrm{N}$, $115^{\circ} 56^{\prime} \mathrm{E}, 1010 \mathrm{~m}, 10-11 . x .1986$, J. Huisman $1 \delta^{\star}$ (RMNH), 1 ๙ิ (UMSP).

Despite the several apparent discrepancies between the specimen figured here and the illustration provided with the type description, we are confident of its determination. The form of the inferior appendages, in ventral view, and the dorsolateral projections from the posterior margin of segment IX are so characteristic and distinctive for the species that it could hardly be anything else. Chimarra thaumas is a very distinctive member of the Chimarra tsudai group, with several characters that easily diagnose it. The apices of the inferior appendages are especially diagnostic because each appendage has paired, acute, apicomesal projections. Segment VIII is also distinctive in being synsclerous, widened dorsally, and with a very deep posteromesal emargination that lacks accompanying spine-like processes. Other diagnostic features include the much enlarged and complex phallotremal sclerite complex and the structure of tergum X, which has short lateral lobes, and divided mesal lobes, the lateral-most of which is heavily sclerotized, bulbous in shape, and with a mucronate apex.

Adult. Color (in alcohol) yellowish-brown. Length of forewing: male $6.0-6.3 \mathrm{~mm}$. Forewing venation: stem of Rs curved, slightly sclerotized and thickened at inflection; fork at base of discal cell somewhat thickened, nearly symmetrical, length of discal cell about 4 times width; 1st fork proximal to crossvein $s ; m$ crossvein proximal to crossveins $s$ and $r-m$, crossvein $s$ hyaline; $2 \mathrm{~A}$ vein not intersecting $3 \mathrm{~A}$ (apparently looped to 1A). Postocular parietal sclerite short. Maxillary palps moderately elongate, segment 3 subequal to 2 and 5 . Protarsal claws of male enlarged, asymmetrical in shape and length.

Male genitalia. Abdominal segment VIII moderate in length; tergum distinctly longer than sternum, unmodified, with V-shaped mesal excavation; sternum VIII without posteroventral projection. Abdominal segment IX moderate in length, with rounded, subspinose projections from the posterodorsal margin, tergum obsolete dorsomesally, without distinct anterolateral apodemes; anteroventral margin in lateral view slightly produced, rounded; posterior margin slightly produced; ventral process absent or nearly so. Preanal appendage setose, short, length about twice width. Inferior appendage longer than tergum X; in lateral view straight, nearly uniformly wide, apex rounded; in ventral view nearly straight, slightly mesally curved apically, mesal surface with paired, acute preapical projections. Tergum $\mathrm{X}$ with sclerotized lateral lobes and separate mesal lobes, mesal membrane lacking; lateral lobe very short, in lateral view simple, slightly displaced ventrally, lobe bearing multiple sensilla; mesal lobe short, distinctly sclerotized, bifid, lateral branch rounded, mucronate, mesal branch digitate, slightly dorsally curved with several apical sensilla. Phallobase tubular, with pronounced basodorsal expansion, moderately elongate, 


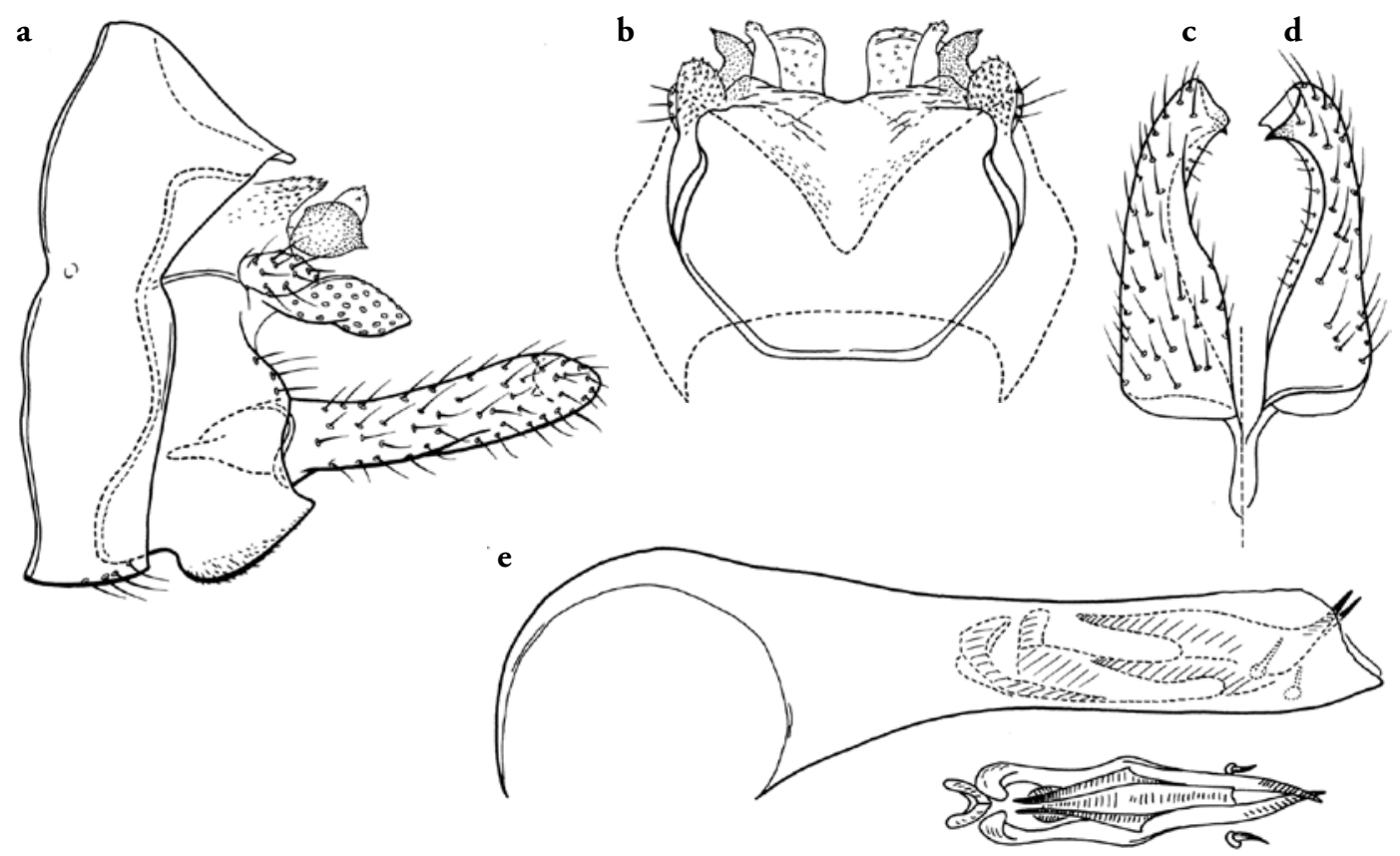

Fig. 33. Chimarra thaumas, male genitalia. - a, lateral; b, segment IX and tergum $\mathrm{X}$, dorsal; dashed line, segment VIII; c, inferior appendage, ventral; d, inferior appendage, dorsal; e, phallic apparatus, lateral; inset, phallotremal sclerite complex, dorsal.

ventral apex weakly sclerotized, slightly projecting. Endotheca length not discernable (not expanded). Phallotremal sclerite complex composed of elongate sclerotized structure, more than half length of phallotheca, apicolaterally with 2 small tack-like spines.

\section{Chimarra vantoli sp. $\mathbf{n}$.}

Fig. 34

Type material. Holotype: $\delta$, Malaysia, Sabah, Crocker Range, $40 \mathrm{~km} \mathrm{~S}$ of Kota Kinabalu, Sinsuron Rd., 1500 m, 18.xii.1989, J. Huisman, (UMSP000107245) (RMNH).

Paratypes. Malaysia, Sabah, same data as holotype, 1 to (UMSP); Kinabalu National Park, Headquarters, Sg. Silau-Silau, 11.viii.2005 \& Sungai Liwagu, 26.ii.2006, W. Mey, 60ิ (UMHU); Kinabalu National Park, Silau-Silau, $06^{\circ} 00^{\prime} \mathrm{N}, 116^{\circ} 32^{\prime} 12^{\prime}$ E, $1560 \mathrm{~m}$, bridge near Bukit Burung trail, 2021.xi.1986, J. Huisman, 2 đิ (UMSP).

Chimarra vantoli belongs to the distinctive subgroup of the Chimarra tsudai group in which tergum VIII is highly modified, invaginated mesally and variously armed with spine-like projections. Generalized features it possesses are a phallotremal sclerite that is not greatly enlarged, and an endotheca that is relatively short and lacks distinctive spines, although the endotheca is "textured" with minute spines. It is most similar to a group of species from Borneo, including C. noloyan, sp. n., C. preapicalis, sp. n., C. physanoton, sp. n., and C. silausilau, sp. n., all of which have similarly shaped inferior appendages, which are short, linear, with acute projections located apicomesally and a tergum VIII that is narrow and modified with variable lengthed spines. As in other members of this group, details in the structure of the inferior appendages and lobes of tergum $\mathrm{X}$ are important for distinguishing species. Chimarra vantoli is most similar to C. physanoton and C. silausi$l a u$, and best distinguished by its nearly subquadrate inferior appendages, with the apicomesal projections neither posteriorly directed nor set off by an apical notch. Other details distinguishing $C$. vantoli are the apices of the lateral lobes of tergum $X$, which are more acute than $C$. silausilau, but less distinctively so than C. physanoton. In the material examined, there was some variation in the development of the spined processes of tergum VIII, either with the paired 
a
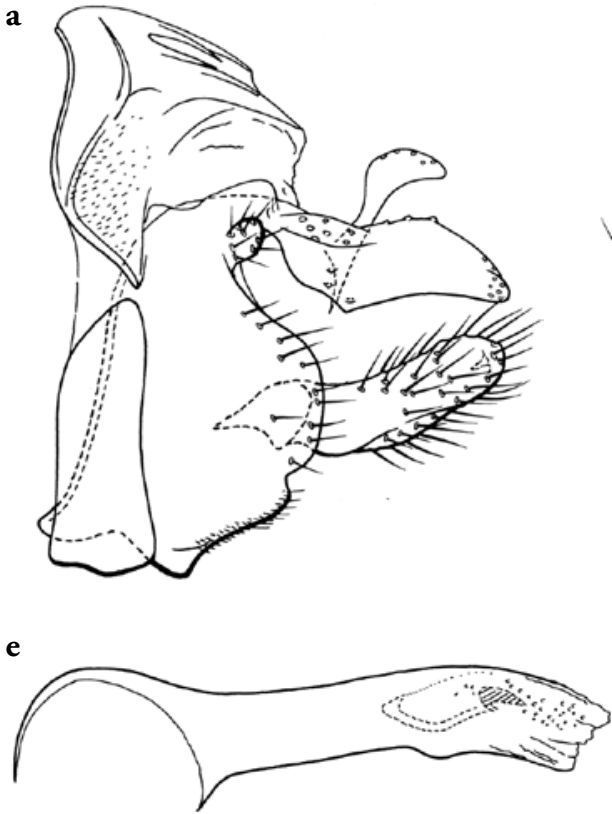

b
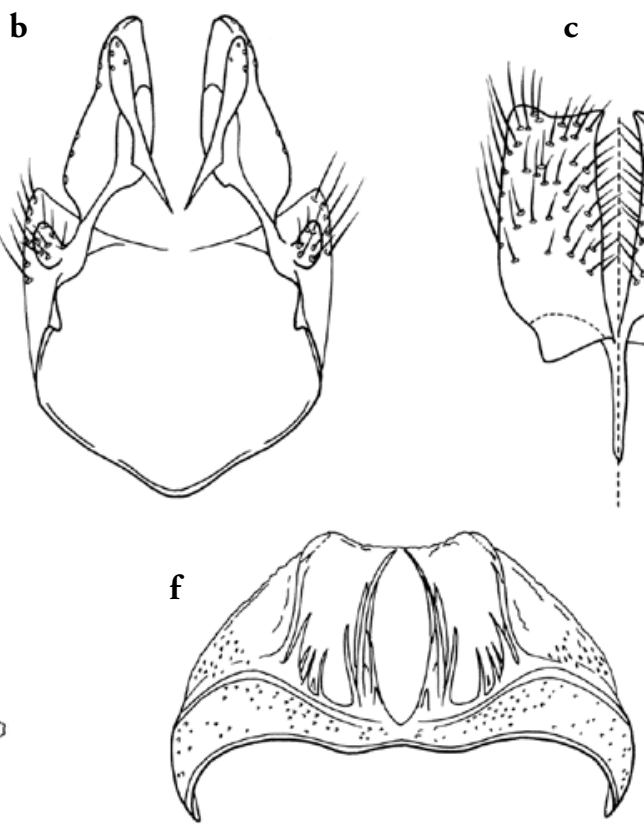

c d
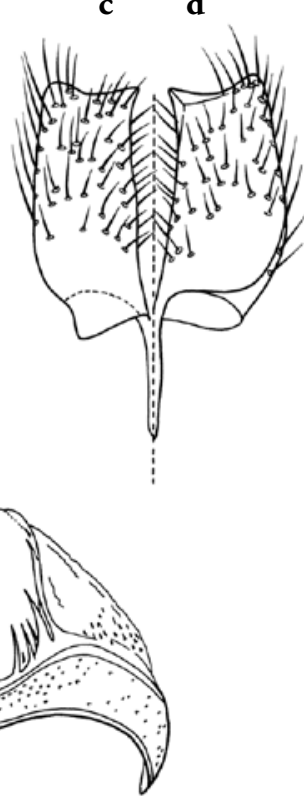

Fig. 34. Chimarra vantoli, male genitalia. - a, lateral; b, segment IX and tergum X, dorsal; c, inferior appendage, ventral; d, inferior appendage, dorsal; e, phallic apparatus, lateral; f, tergum VIII, dorsal.

processes composed of variable lengthed spines, as in the figure of the holotype specimen, or with each of the paired processes having short lateral spines along its length.

Adult. Color (in alcohol) light brown. Length of forewing: male $5.9-6.3 \mathrm{~mm}$, female $6.4-6.6 \mathrm{~mm}$. Forewing venation: stem of Rs curved, distinctly sclerotized and thickened at inflection; fork at base of discal cell distinctly thickened, nearly symmetrical, length of discal cell about 2.5 times width; $m$ crossvein proximal to crossveins $s$ and $r$ - $m$, crossvein $s$ not hyaline; $2 \mathrm{~A}$ vein not intersecting $3 \mathrm{~A}$ (apparently looped to 1A). Postocular parietal sclerite moderately elongate. Maxillary palps moderately elongate, segment 3 distinctly longer than 2 , 3 subequal to 5. Protarsal claws of male enlarged, asymmetrical in shape and length.

Male genitalia. Abdominal segment VIII short; tergum about as long as sternum, modified, with mesal excavation, excavation with elongate laciniate fringe divided into 2 branches, sometimes with short lateral fringe along each of the 2 branches; sternum VIII without posteroventral projection. Abdominal segment IX relatively wide dorsolaterally, tergum obsolete dorsomesally, without distinct anterolateral apodemes; anteroventral margin in lateral view not, or scarcely produced; posterior margin sinuously produced; ventral process projecting ventrally, very short, broad basally, subtriangular. Preanal appendage setose, very small, rounded. Inferior appendage about as long as tergum $\mathrm{X}$; in lateral view straight, apex rounded; in ventral view nearly straight, apex subtruncate, with apicomesal tooth-like projection. Tergum X with sclerotized lateral lobes and separate mesal lobes, mesal membrane lacking; lateral lobe moderately long, in lateral view apex narrow, slightly downcurved, lobe bearing multiple sensilla; mesal lobe elongate, digitate, posterodorsally directed, apex enlarged. Phallobase tubular, with pronounced basodorsal expansion, short. Endotheca relatively short, with granularly textured region; without endothecal spines. Phallotremal sclerite complex composed of rod and ring structure, rod short, apically with pair of short, curved, lateral sclerites.

Etymology. Named for Dr. Jan van Tol, National Museum of Natural History Naturalis, friend and colleague of J. Huisman.

\section{Chimarra vanwelzeni sp. $\mathbf{n}$.}

Fig. 35

Type material. Holotype: $\hat{0}$, Malaysia, Sabah, Crocker Range, $40 \mathrm{~km} \mathrm{~S}$ of Kota Kinabalu, Sinsuron Rd., 1500 m, 18.xii.1989, J. Huisman, (UMSP000107158) (RMNH). 
a

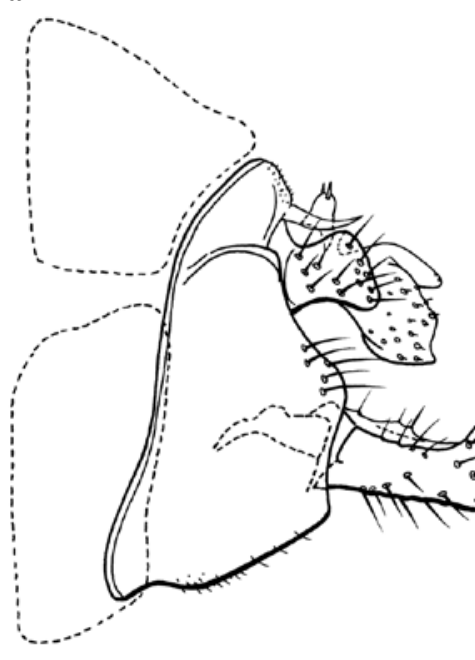

b

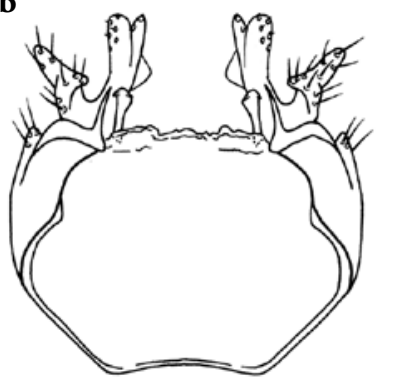

d

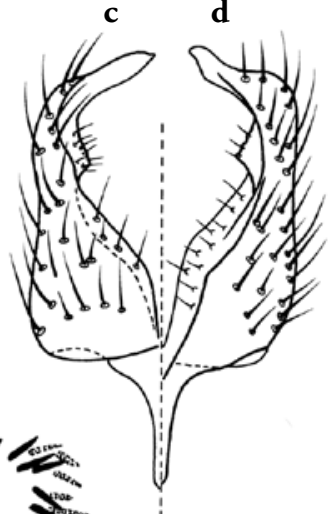

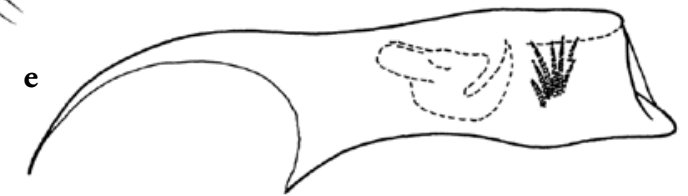

Fig. 35. Chimarra vanwelzeni, male genitalia. - a, lateral; b, segment IX and tergum X, dorsal; c, inferior appendage, ventral; $d$, inferior appendage, dorsal; e, phallic apparatus, lateral; inset, phallotremal sclerite and endothecal spines, dorsal.

Paratypes. Malaysia, Sabah, Kinabalu National Park, Headquarters, roadside, $06^{\circ} 00^{\prime} \mathrm{N}, 116^{\circ} 32^{\prime} \mathrm{E}$, 1500 m, 12.v.1987, J. Huisman, $10^{\Uparrow}$ (UMSP); West Coast, Gn. Kinabalu, roadside, 1500 m, 26.xii.1989, J. Huisman, $1 \delta^{\Uparrow}$ (UMSP).

Chimarra vanwelzeni does not share an evident similarity to any other species of the Chimarra tsudai group from Borneo. It is distinctive in the shape of its inferior appendages, which have their apices strongly narrowed and mesally curved in ventral view. The enlarged, short, sclerous phallotremal sclerite is also distinctive, as are the very short and subdivided lateral lobes of tergum X.

Adult. Color (in alcohol) light brown. Length of forewing: male 7.5-7.8 mm. Forewing venation: stem of Rs curved, distinctly sclerotized and thickened at inflection; fork at base of discal cell somewhat thickened, nearly symmetrical, length of discal cell about 4 times width; $m$ crossvein proximal to crossveins $s$ and $r-m$, crossvein $s$ not hyaline; $2 \mathrm{~A}$ vein not intersecting 3A (apparently looped to $1 \mathrm{~A}$ ). Postocular parietal sclerite moderately elongate. Maxillary palps elongate, segment 3 slightly greater than 2, 3 subequal to 5 . Protarsal claws of male enlarged, asymmetrical in shape and length.

Male genitalia. Abdominal segment VIII moderate in length; tergum longer than sternum, unmodified; sternum VIII without posteroventral projection.
Abdominal segment IX relatively wide dorsolaterally, tergum obsolete dorsomesally, without distinct anterolateral apodemes; anteroventral margin in lateral view not, or scarcely produced; posterior margin distinctly produced; ventral process absent or nearly so. Preanal appendage setose, relatively large, subquadrate. Inferior appendage much longer than tergum X; in lateral view slightly curved, nearly uniformly wide, narrow distally, apex acute; in ventral view nearly straight basally, mesally curved apically, apex elongate, narrow, strongly posteromesally bent. Tergum X with sclerotized lateral lobes and separate mesal lobes, mesal membrane lacking; lateral lobe short, bifurcate, in lateral view lateral lobe bifurcate into a simple, obovate, strongly downcurved branch and short, digitate mesal branch, lobe bearing multiple sensilla; mesal lobe short, digitate, distinctly sclerotized, dorsally directed, apex with approximately 2 short spine-like projections. Phallobase tubular, with pronounced basodorsal expansion, relatively short. Endotheca relatively short; with patch of short endothecal spines. Phallotremal sclerite complex composed of short, highly modified rod and ring structure, with distinctly sclerotized with bifurcating apical branches.

Etymology. Named for Dr. Peter van Welzen, National Herbarium of The Netherlands, Leiden Branch, University of Leiden, friend of J. Huisman. 
a

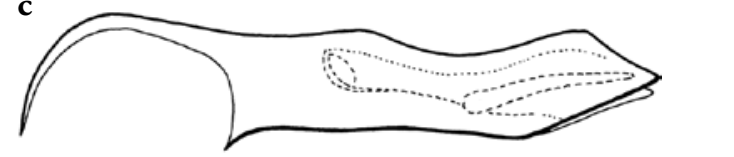

b
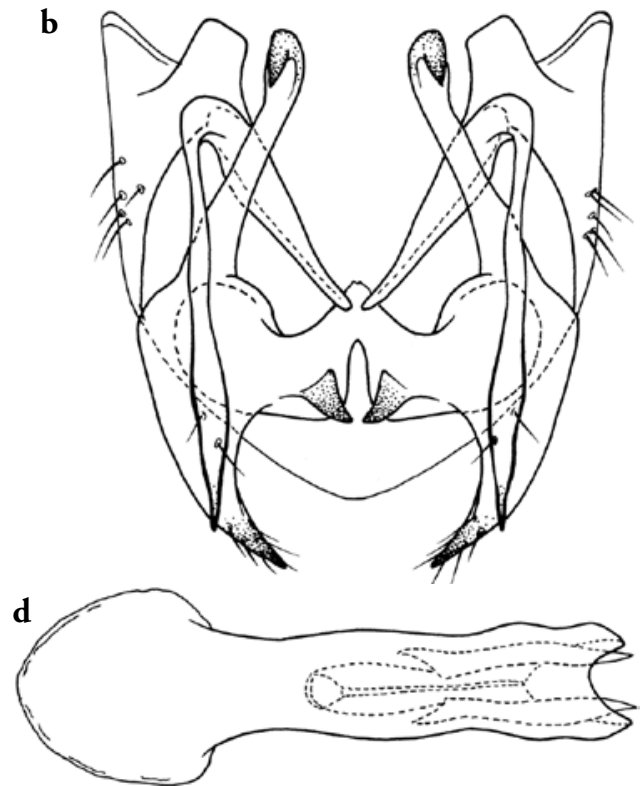

Fig. 36. Chimarra noohi, male genitalia. - a, lateral; b, segment IX and inferior appendages, caudal; c, phallic apparatus, lateral; $\mathrm{f}$, phallic apparatus, dorsal.

\section{Unplaced species}

\section{Chimarra noohi sp. $\mathbf{n}$.}

Figs 36, 44

Type material. Holotype: $\delta$, Malaysia, Sabah, Long $\mathrm{Pa} \mathrm{Sia}$, confluence Sg. Pa Sia \& Sg. Matang, $04^{\circ} 24^{\prime} \mathrm{N}, 115^{\circ} 43^{\prime} \mathrm{E}, 1000 \mathrm{~m}, 10$.iv.1987, J. Huisman (UMSP000107195) (RMNH).

Chimarra noohi is a most unusual species of Chima$\mathrm{rra}$. The shape of segment IX, development of the lateral lobes of tergum $\mathrm{X}$, and the shape of the inferior appendages are all distinctive and diagnostic. That the elongate, hornlike processes on the dorsolateral margin of segment IX are lateral lobes of tergum X is suggested by its 2 short setae (or seta-like sensilla). A somewhat similar development of tergum X, also with seta-like projections is found in Chimarra furti Mey, 1998 from the Philippines and also in Edidiehlia hiskia Malicky, 1993 from Sumatra, although the latter has 4 setae on each lobe (based on the illustration provided). Venational characters, forewing with the $s, r-m$, and $m$ veins unpigmented and nearly linearly arranged, Rs vein of the forewing lacking the typical curvature found in most species of the subgenus Chimarra, and also the absence of an anal loop in the hind wing and the fused Sc and R1 of the hind wing are similar to Edidiehlia. Venational characters of C. furti were not provided with its description. Similar venational characters, except for the absence of an anal loop are found in other species in the subgenus Chimarra, notably the Chimarrhafra group from Africa and C. uvana Kimmins, 1957 from Sri Lanka. Another species described below, C. ventritropis, sp. $\mathrm{n}$. also lacks an anal loop in the hind wing, but in other respects bears no resemblance to $C$. noohi or the other 2 species discussed. A single foretibial spur provides the character evidence for placing C. noohi in the genus Chimarra and the divided lobes of tergum $\mathrm{X}$ for placing it in the subgenus Chimarra. The same evidence would apply to Edidiehlia hiskia, but because both it and $C$. furti are so unusual and different from one another, as well as from C. noohi, and because we have not examined these other species, we are not proposing any nomenclatural changes.

Adult. Color (in alcohol) yellowish-brown. Length of forewing: male $3.4 \mathrm{~mm}$. Forewing venation: stem of Rs nearly straight; fork at base of discal cell somewhat thickened, nearly symmetrical, length of discal cell about 2.5 times width; $m$ crossvein aligned with crossveins $s$ and $r-m$, crossvein $s$ hyaline; $2 \mathrm{~A}$ vein intersecting 3A vein (apparently forked apically). Postocular parietal sclerite short. Maxillary palps relatively short, segment 3 slightly longer than 3, 5 slightly longer than 3 . Protarsal claws of male not or very little enlarged, symmetrical. 


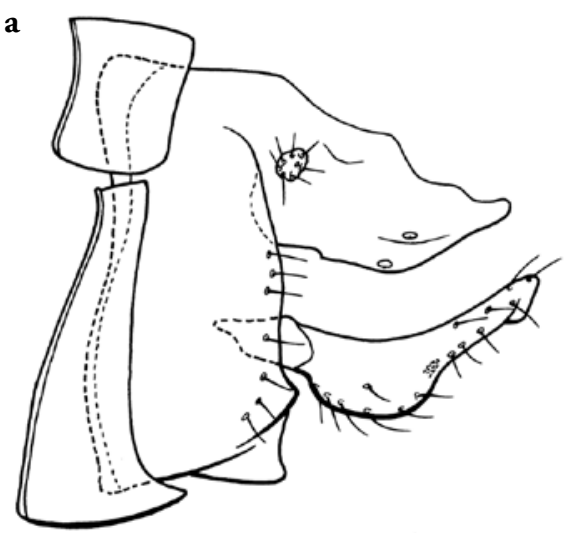

b
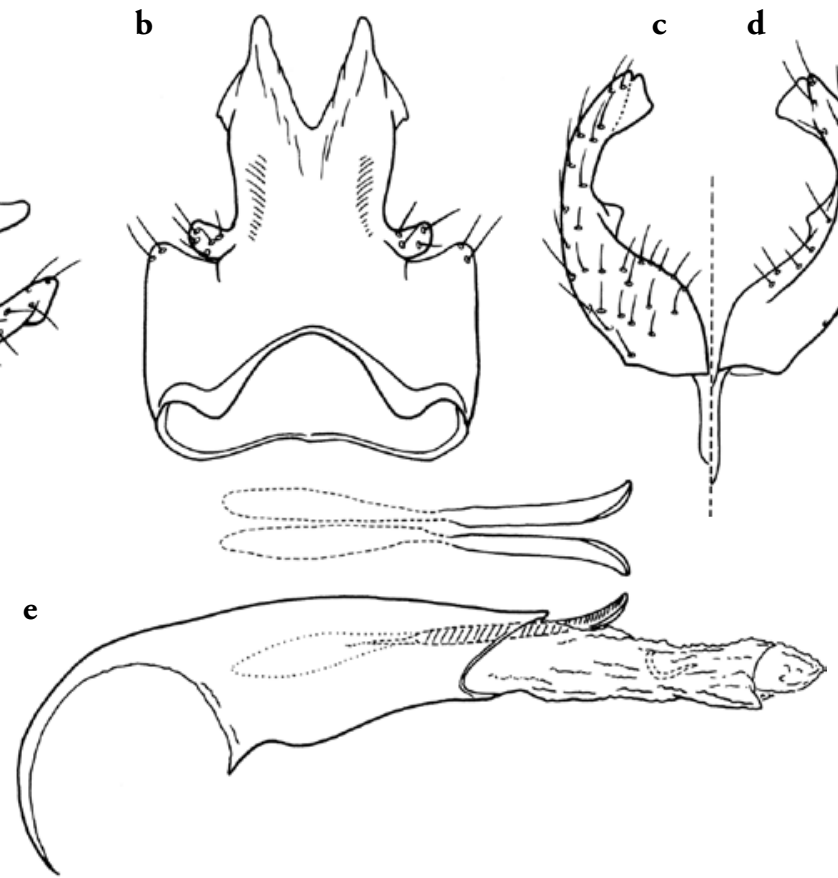

Fig. 37. Chimarra polyneikes, male genitalia. - a, lateral; b, segment IX and tergum X, dorsal; c, inferior appendage, ventral; $\mathrm{d}$, inferior appendage, dorsal; e, phallic apparatus, lateral; inset, endothecal spines, dorsal.

Male genitalia. Abdominal segment VIII short; tergum about as long as sternum, unmodified; sternum VIII with slight posteroventral projection. Abdominal segment IX nearly trianguloid in lateral view, wide ventrally, narrowing dorsally, tergum obsolete dorsomesally, with pair of short apodemes from anterolateral margin; ventral process absent or nearly so. Preanal appendage apparently absent. Inferior appendage about as long as tergum $\mathrm{X}$; in lateral view with acute sclerotized ventral apex and reclinate dorsal process, narrowing to an acute, posteriorly curved dorsal apex; mesal surface with acute, sclerotized, tooth-like projection. Tergum X with sclerotized lateral lobes only, mesal membrane lacking; lateral lobe elongate, narrow, apex acute, in lateral view arched at base and strongly ventrally curved, lobe bearing 2 short setae or seta-like sensilla, preapically. Phallobase tubular, with pronounced basodorsal expansion, moderately elongate, slightly invaginated apicoventrally. Phallotremal sclerite complex composed of elongate sclerotized structure more than half length of phallobase, complex apparently consisting of elongate rod and ring structure, with accompanying pair of elongate, symmetrical, apicolateral sclerites.

Etymology. Named for Nooh Bin Dawa, in recognition of the help and friendship given to J. Huisman during her studies and collecting on Borneo.

\section{Chimarra polyneikes Malicky}

Figs 37, 45

Chimarra polyneikes Malicky, 2008: 840, holotype ô, Indonesia (Eastern Kalimantan), Zoologische Museum Lausanne.

Material examined. Malaysia, Sabah, Poring Hot Spring, $12 \mathrm{~km}$ NNE Ranau, Sg. Kepungit, E park boundary, $06^{\circ} 03^{\prime} \mathrm{N}, 116^{\circ} 42^{\prime} \mathrm{E}, 480 \mathrm{~m}$, 26.xi.1986, J. Huisman $1 \hat{o}^{\top}(\mathrm{RMNH}) ; 12 \mathrm{~km}$ NNE Ranau, Poring Hot Spring, Sg. Tananansad, $06^{\circ} 03^{\prime} \mathrm{N}, 116^{\circ} 42^{\prime} \mathrm{E}, 560 \mathrm{~m}, 28$.viii.1986, J. Huisman, $40^{\star}$ (UMSP), $20^{\star}$ (RMNH).

Chimarra polyneikes has a simple morphology that might deflect attention from its very unusual combination of characters. The shape of the inferior appendages, with their chisel-like apices is diagnostic and distinctive. The general form of the inferior appendages, as well as the presence of 2 sensilla on the lateral lobes of tergum $\mathrm{X}$ and 2 endothecal spines in the phallic apparatus suggest a relationship to the Chimarra digitata group. However, the shape of segment IX, without a projecting anteroventral margin, without pronounced anterodorsal apodemes, and with a ventral process more ventrally than posteriorly directed, would be unusual characters for a species in the Chimarra digitata group. 

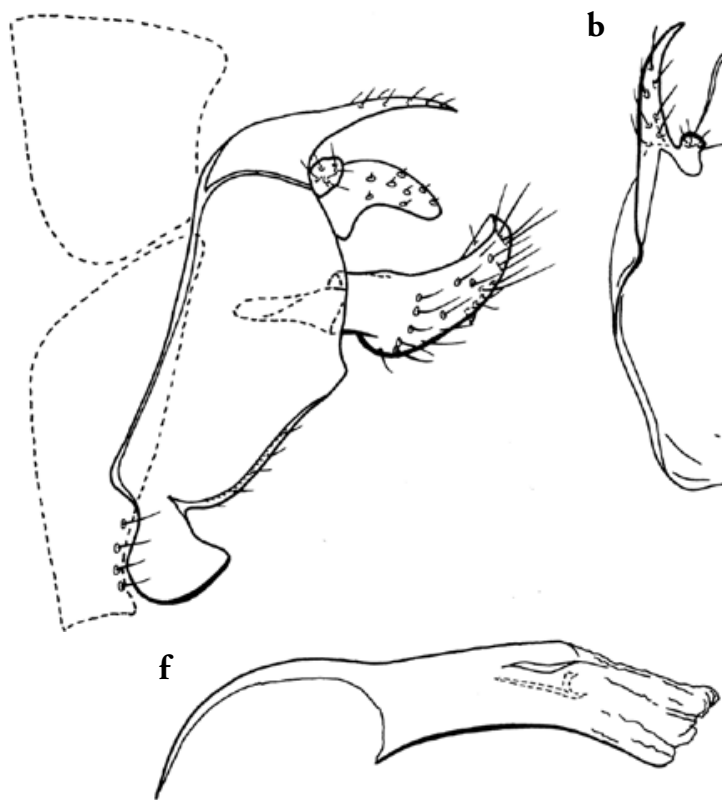

b

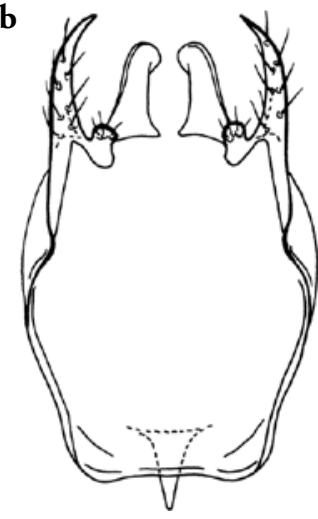

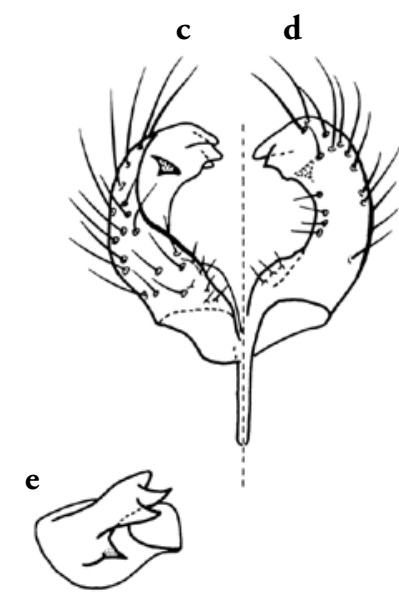

d

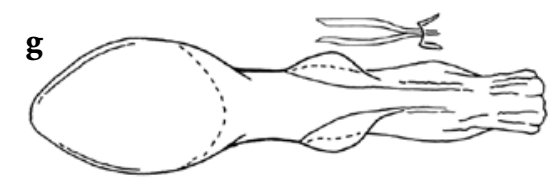

Fig. 38. Chimarra ventritropis, male genitalia. - a, lateral; b, segment IX and tergum X, dorsal; c, inferior appendage, ventral; $d$, inferior appendage, dorsal; e, inferior appendage, caudal; $f$, phallic apparatus, lateral; g, phallic apparatus, dorsal; inset, phallotremal sclerite, dorsal.

Additionally, the lateral lobes of tergum $\mathrm{X}$ are incompletely divided dorsomesally, possibly a secondarily derived state and not reflective of a basal position in the subgenus Chimarra as a whole. Venational characters for $C$. polyneikes are primitive, with the Rs vein of the forewing nearly straight, and the $s, r-m$, and $m$ veins linear and hyaline. Like members of the Chimarra tsudai group, the $2 \mathrm{~A}$ vein of the forewing is looped to the $1 \mathrm{~A}$ vein. This combination of characters does not suggest a clear relationship to either of the main species lineages discussed in this paper.

Adult. Color (in alcohol) yellowish-brown. Length of forewing: male $3.4-3.6 \mathrm{~mm}$. Forewing venation: stem of Rs nearly straight; fork at base of discal cell distinctly thickened, nearly symmetrical, length of discal cell about 3 times width; 1st fork slightly proximal to $s$ crossvein; $m$ crossvein aligned with crossveins $s$ and $r-m$, crossvein $s$ hyaline; $2 \mathrm{~A}$ vein not intersecting 3A (apparently looped to 1A). Hind wing venation: $S c$ and R1 fused, first fork absent. Postocular parietal sclerite short. Maxillary palps relatively short, segment 3 slightly longer than 2, 3 subequal to 5 . Protarsal claws of male not or very little enlarged, symmetrical.

Male genitalia. Abdominal segment VIII short; tergum about as long as sternum, unmodified, entire, without mesal excavation. Abdominal segment IX relatively wide dorsolaterally, tergum relatively long dorsally, continuous with base of tergum X, without distinct anterolateral apodemes; anteroventral margin in lateral view not, or scarcely produced; posterior margin nearly straight; ventral process projecting ventrally, broad basally, subtriangular. Preanal appendage setose, small, rounded, slightly narrowed basally. Inferior appendage about as long as tergum X; in lateral view, dorsally inflected basoventrally, widest basally, narrow distally, basoventral margin rounded, apex subquadrate; in ventral view mesally curved, mesal surface with small, tooth-like projection at midlength. Tergum $\mathrm{X}$ lightly sclerotized dorsally, apically cleft to from lateral lobes; lateral lobe moderately long, with apex narrowed, subacute, lobe bearing 2 sensilla, sensilla at about midlength, on slightly projecting, lateral protrusions. Phallobase tubular, with pronounced basodorsal expansion, moderately elongate, ventral margin slightly bulged basally. Endotheca elongate, tubular, granularly textured region not discernable; with 2 elongate endothecal spines, nearly symmetrical in length and position, apices distinctly flattened. Phallotremal sclerite complex composed of rod and ring structure, rod short. 


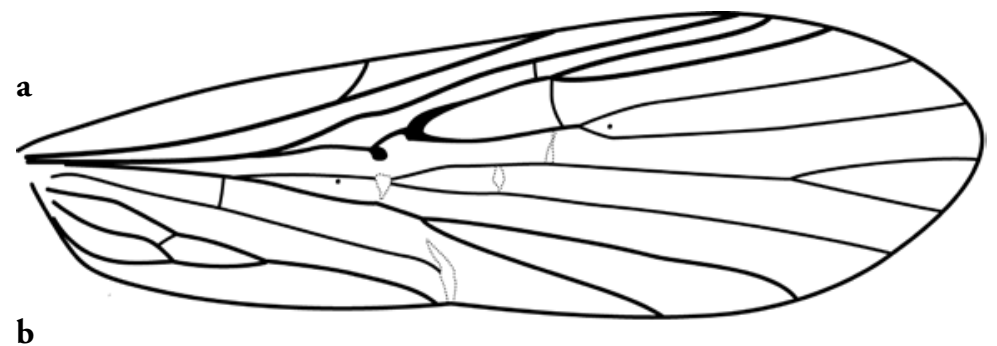

b
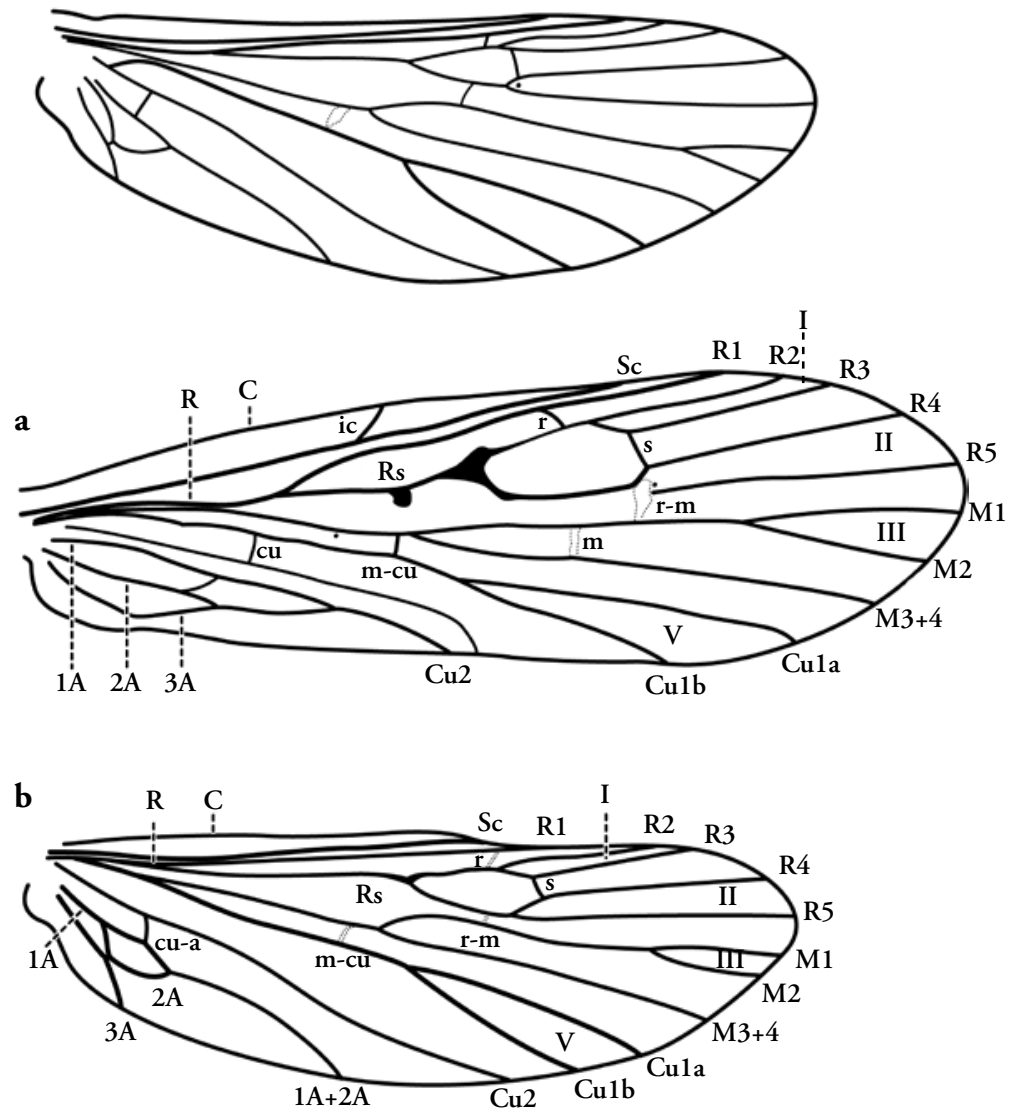

Fig. 39. Chimarra devogeli, wings. - a, forewing; $b$, hind wing.

Fig. 40. Chimarra phlegyas, wings. $-a$, forewing; $b$, hind wing.

\section{Chimarra ventritropis sp. $\mathbf{n}$.}

Figs 38, 46

Type material. Holotype: đิ, Malaysia, Sabah, Kinabalu National Park, Headquarters, Sg. Liwagu crossing Silau-Silau trail, $06^{\circ} 00^{\prime} \mathrm{N}, 116^{\circ} 33^{\prime} \mathrm{E}$, 1470 m, 15.xi.1986, J. Huisman (UMSP000107152) (RMNH).

Paratypes. Malaysia, Sabah, Kinabalu National Park, Sg. Liwagu, 1100 m, 12.viii.2005, W. Mey, 50 (UMHU); Crocker Range, $40 \mathrm{~km} \mathrm{~S}$ of Kota Kinabalu, Sinsuron Rd., 1500 m, 20.xii.1989, J. Huisman, 1 ô (UMSP).
Chimarra ventritropis is a distinctive and unusual species of Chimarra, easily diagnosed by the distinctive ventral process of segment IX, dorsal spine-like projections of segment IX, and the shape of the inferior appendages. Like species in the Chimarra tsudai group, it has multiple sensilla on the lateral lobes of tergum X, but lacks the sclerotized, digitate mesal lobes that characterize that group. The overall set of characters it possesses suggests that it may represent a species or lineage basal to the Chimarra tsudai group.

Adult. Color (in alcohol) light brown. Length of forewing: male $4.8-5.4 \mathrm{~mm}$. Forewing venation: 


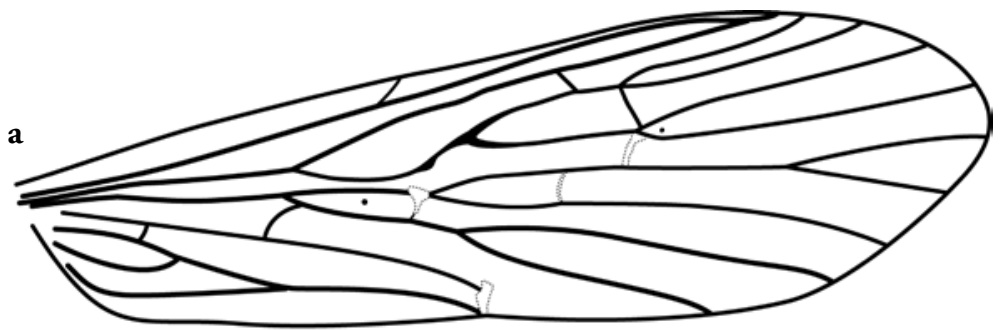

Fig. 41. Chimarra jannekae, wings. - a, forewing; $b$, hind wing.

b
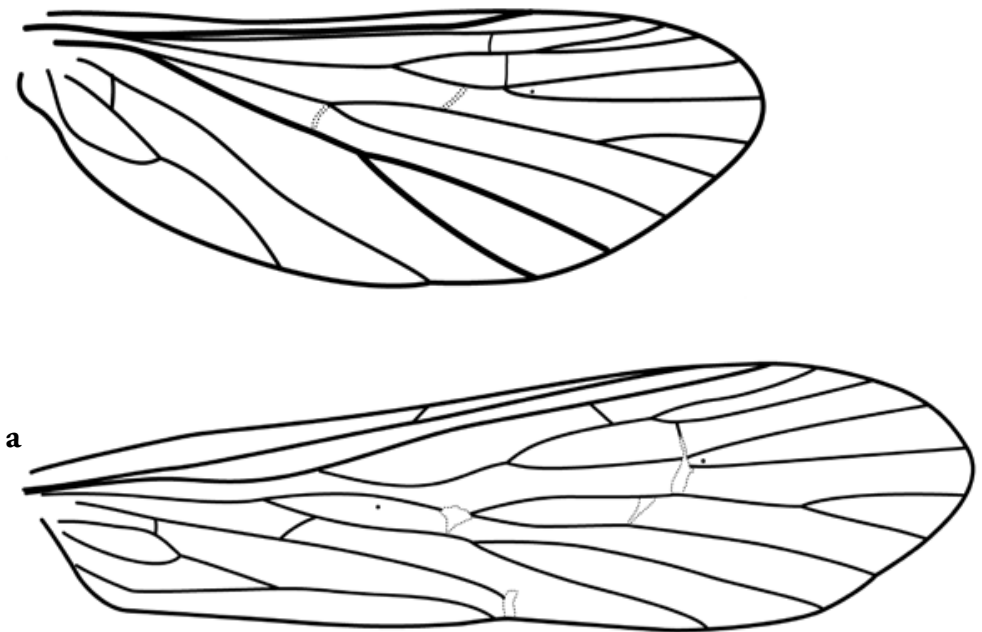

Fig. 42. Chimarra phillipsae, wings. $-a$, forewing; $b$, hind wing.

b
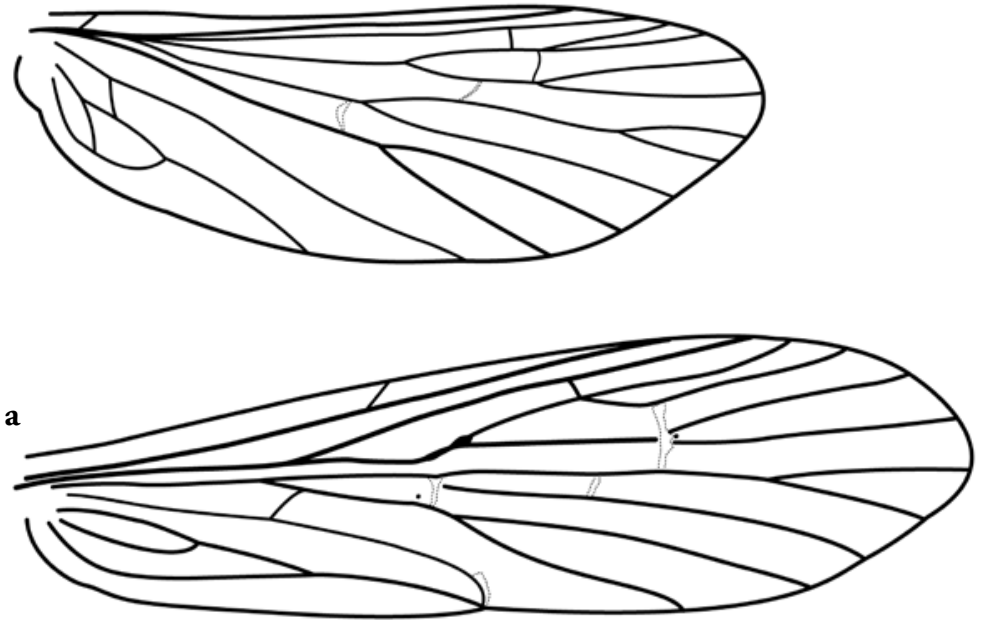

Fig. 43. Chimarra thaumas, wings. $-a$, forewing; $b$, hind wing.

b

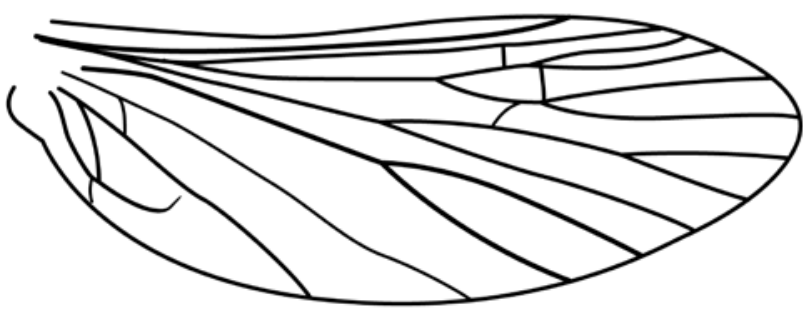




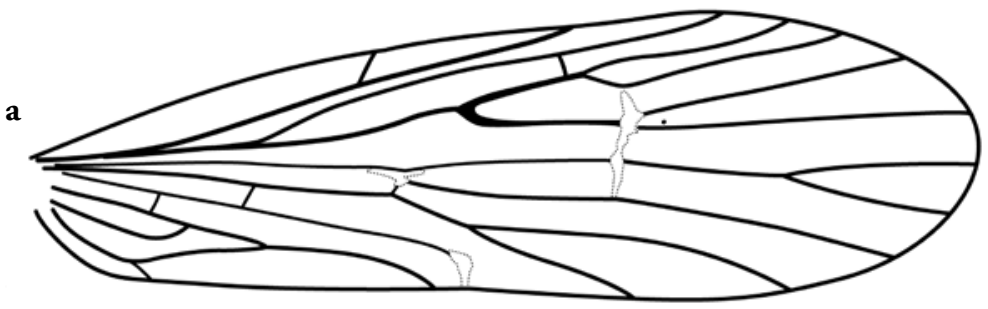

b
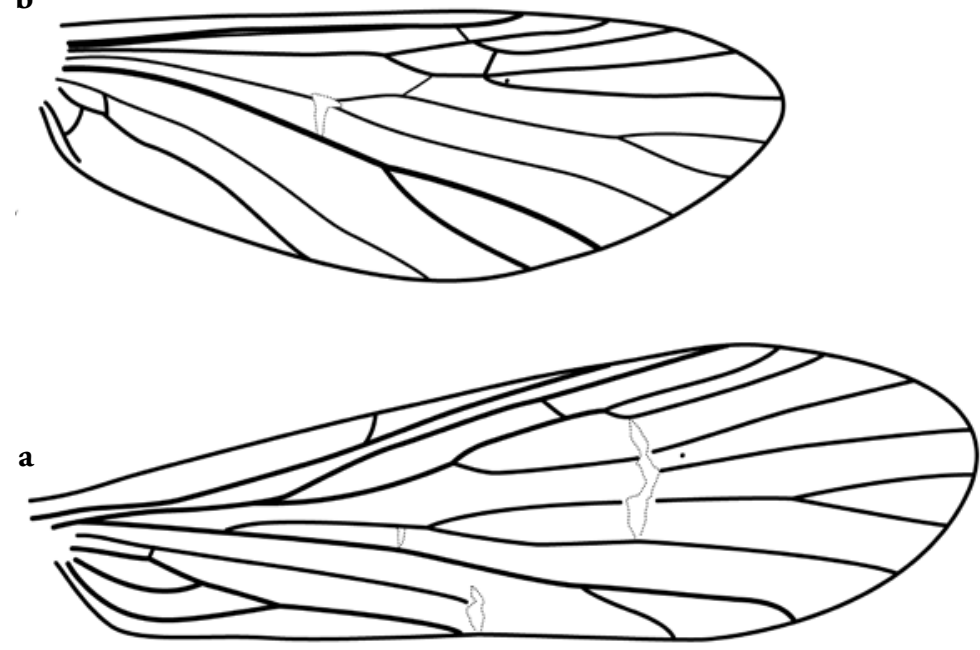

b
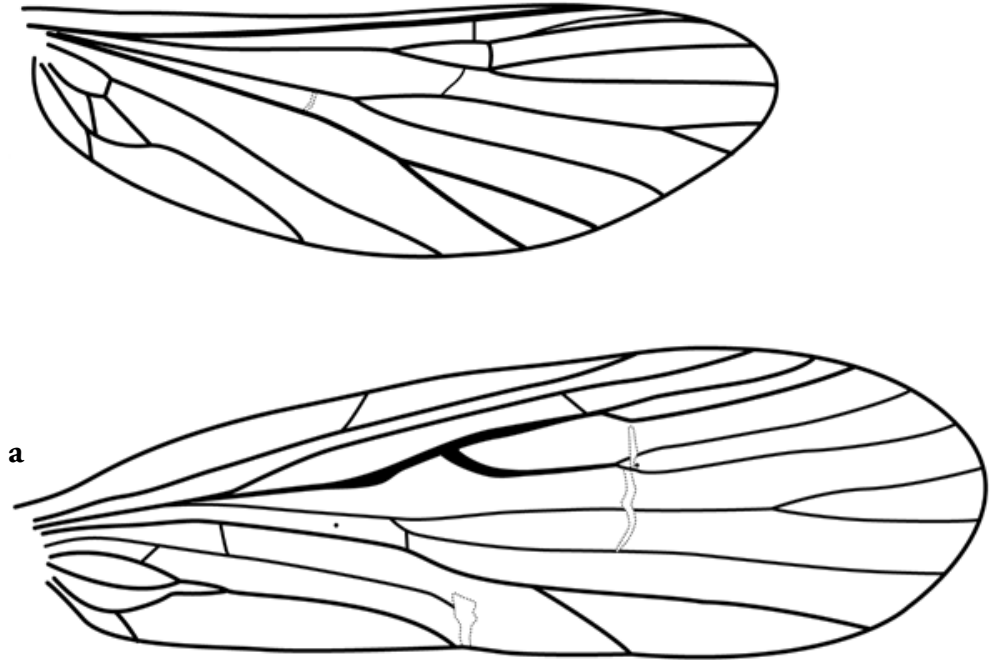

b

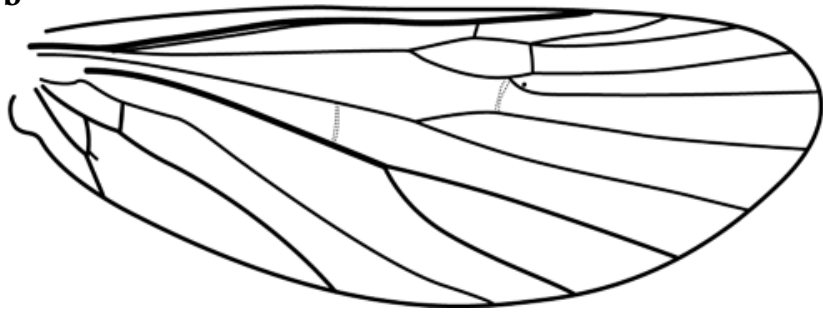

Fig. 44. Chimarra noohi, wings. $-a$, forewing; $b$, hind wing.

Fig. 45. Chimarra polyneikes, wings. $-a$, forewing; $b$, hind wing.

Fig. 46. Chimarra ventritropis, wings. - a, forewing; $\mathrm{b}$, hind wing. 
stem of Rs only slightly curved; fork at base of discal cell somewhat thickened, nearly symmetrical, length of discal cell about 3 times width; $m$ crossvein aligned with crossveins $s$ and $r-m$, crossvein $s$ hyaline; $2 \mathrm{~A}$ vein not intersecting 3A (apparently looped to 1A). Postocular parietal sclerite short. Maxillary palps elongate, segment 3 distinctly longer than 2, 5 longer than 3. Protarsal claws of male not or very little enlarged, symmetrical.

Male genitalia. Abdominal segment VIII moderate in length; tergum distinctly longer than sternum, unmodified; sternum VIII without posteroventral projection. Abdominal segment IX relatively wide dorsolaterally, tergum obsolete dorsomesally, without distinct anterolateral apodemes; anteroventral margin in lateral view not, or scarcely produced; posterior margin slightly produced; ventral process projecting ventrally, forming ventrally rounded, posteriorly subacute lobe, somewhat constricted basally. Preanal appendage setose, small, rounded, slightly narrowed basally. Inferior appendage longer than tergum X; in lateral view dorsally inflected basoventrally, nearly uniformly wide, basoventral margin rounded, apex subquadrate, with short apical and preapical teeth; in ventral view strongly mesally curved. Tergum $\mathrm{X}$ with sclerotized lateral lobes only, mesal membrane lacking; lateral lobe very short, in lateral view simple, rounded apically, slightly downcurved, lobe bearing multiple sensilla. Phallobase tubular, with pronounced basodorsal expansion, short, preapically with dorsolateral flange-like expansions. Endotheca length not discernable (not expanded); without endothecal spines. Phallotremal sclerite complex composed of rod and ring structure, rod very short, preapically with short lateral sclerites.

Etymology. This species is named C. ventritropis, a noun in apposition, from the Latin words venter, belly, and tropis, a keel, and referring to the distinctive ventral process of segment IX in this species.

\section{Acknowledgments}

The fieldwork of Jolanda Huisman in East Malaysia was made possible through the financial support of the Melchior Treub Foundation, the Uyttenboogaart-Eliasen Foundation, and the National Museum of Natural History (Leiden, The Netherlands). Jolanda Huisman is very grateful to Anthea PhillipsLamb, Fui-Lian Tan-Inger, Gabriel Sinit, Rienk de Jong, Ed de Vogel, Chan Chu Lun, Anthony Lamb, Peter van Welzen, Jan van Tol, and Nooh Bin Dawa, for their help and support, and to her daughters, Karlijn and Janneke Holzenthal. Her gratitude is expressed in the etymologies of several of the species described above. Gratitude is also extended to
Dr. Wolfram Mey, Museum für Naturkunde, Humbolt-Universität, Berlin for checking the utility of the illustrations and for extending the available records of examined material. We also thank Dr. Erik J. van Nieukerken for his editorial services, useful suggestions, and assistance in finding some of the literature cited. This material is based upon work supported by the National Science Foundation grant no. DEB 0117772. Additional support was provided by the University of Minnesota Insect Collection under projects AES-MIN-17-015 and 17-017. This support is gratefully acknowledged.

\section{References}

Banks, N., 1931. Neuropteroid insects from North Borneo, particularly from Mt. Kinabalu. - Journal of the Federated Malay States Museums 16: 411-429.

Blahnik, R.J., 1998. A revision of the Neotropical species of the genus Chimarra, subgenus Chimarra (Trichoptera: Philopotamidae). - Memoirs of the American Entomological Institute 59: vi+1-318.

Blahnik, R.J. \& R.W. Holzenthal, 2004. Collection and curation of Trichoptera, with an emphasis on pinned material. - Nectopsyche, Neotropical Trichoptera Newsletter 1: 8-20. http://www.entomology.umn. edu/museum/links/news.html [visited on 15 June 2008].

Blahnik, R.J., R.W. Holzenthal \& A. Prather, 2007. The lactic acid method for clearing Trichoptera genitalia. - In: J. Bueno-Soria, R. Barba-Alvarez \& B. Armitage (Eds.), Proceedings of the XIIth International Symposium on Trichoptera: 9-14. The Caddis Press, Columbus, Ohio, USA.

Cartwright, D.I., 2002. The Australian species of Chimarra Stephens (Trichoptera: Philopotamidae). - Memoirs of the Museum of Victoria 59: 393-437.

Chantaramongkol, P. \& H. Malicky, 1989. Some Chimarra (Trichoptera: Philopotamidae) from Thailand. - Aquatic Insects 11: 223-240.

Kimmins, D.E., 1955. Results of the Oxford University expedition to Sarawak, 1934. Order Trichoptera. - Sarawak Museum Journal (New Series) 6: 374-442.

Malicky, H., 1989. Köcherfliegen (Trichoptera) von Sumatra und Nias: Die Gattungen Chimarra (Philopotamidae) und Marilia (Odontoceridae), mit Nachträgen zu Rhyacophila (Rhyacophilidae). - Mitteilungen der Schweizerischen Entomologischen Gesellschaft 62: 131-143.

Malicky, H., 1993. Neue asiatische Köcherfliegen (Trichoptera: Rhyacophilidae, Philopotamidae, Ecnomidae und Polycentropodidae). - Entomologische Berichte Luzern 29: 77-88.

Malicky, H., 1994. Neue Trichopteren aus Nepal, Vietnam, China, von den Philippinen und vom BismarckArchipel (Trichoptera). - Entomologische Berichte Luzern 31: 163-172. 
Malicky, H., 1995. Neue Köcherfliegen (Trichoptera, Insecta) aus Vietnam. - Linzer Biologische Beiträge 27: 851-885.

Malicky, H., 2000. Einige neue Köcherfliegen aus Sabah, Nepal, Indien und China (Trichoptera: Rhyacophilidae, Hydrobiosidae, Philopotamidae, Polycentropodidae, Ecnomidae, Psychomyiidae, Hydropsychidae, Brachycentridae, Odontoceridae, Molannidae). - Braueria 27: 32-39.

Malicky, H. 2008. Köcherfliegen (Insecta, Trichoptera) aus der Umgebung von Malinau (Kalimantan, Borneo, Indonesien). - Linzer Biologische Beiträge 40: 833-879.

Malicky, H. \& P. Chantaramongkol, 1993a. Neue Trichopteren aus Thailand. Teil 1: Rhyacophilidae, Hydrobiosidae, Philopotamidae, Polycentropodidae, Ecnomidae, Psychomyidae, Arctopsychidae, Hydropsychidae. - Linzer Biologische Beiträge 25: 433-487.

Malicky, H. \& P. Chantaramongkol, 1993b. Neue Trichopteren aus Thailand. Teil 2: Rhyacophilidae, Philopotamidae, Polycentropodidae, Ecnomidae, Psychomyidae, Xiphocentronidae, Helicopsychidae, Odontoceridae. - Linzer Biologische Beiträge 25: 1137-1187.

Malicky, H., \& P. Chantaramongkol, 1997. Weitere neue Köcherfliegen (Trichoptera) aus Thailand. - Linzer Biologische Beiträge 29: 203-215.

Malicky, H., \& P. Chantaramongkol, 2003. Vierzehn neue Köcherfliegen aus Thailand (Insecta, Trichoptera). - Linzer Biologische Beiträge 35: 915-925.

Malicky, H, P. Chantaramongkol, P. Bunlue, N. Changthong, J. Nawvong, A. Nuntakwang, T. Prommi,
P. Thamsenanupap \& D. Thapanya, 2004. 27 neue Köcherfliegen aus Thailand (Insecta, Trichoptera). - Linzer Biologische Beiträge 36: 287-304.

Malicky, H., P. Chantaramongkol, P. Chaibu, P. Thamsenanupap \& I. Thani, 2000. Acht neue Köcherfliegen aus Thailand. - Braueria 27: 29-31.

Malicky, H., P. Chantaramongkol, N. Changthong \& P. Thamsenanupap, 2005. Neun neue Köcherfliegen aus Thailand (Trichoptera). - Linzer Biologische Beiträge 37: 597-604.

Malicky, H., P. Chantaramongkol, N. Changthong, A. Nuntakwang \& P. Thamsenanupap, 2006. Beschreibungen einiger Köcherfliegen aus Nord-Thailand (Trichoptera). - Braueria 33: 39-42.

Malicky, H. \& T. Prommi, 2006. Beschreibungen einiger Köcherfliegen aus Süd-Thailand (Trichoptera). - Linzer Biologische Beiträge 38: 1591-1608.

Mey, W., 1998a. Contribution to the knowledge of the caddisfly fauna of the Philippines, III (Insecta: Trichoptera). - Entomofauna 19: 1-32.

Mey, W., 1998b. Die Köcherfliegenfauna des Fan Si PanMassivs in Nord-Vietnam. 3. Beschreibung weiterer neuer Arten (Trichoptera). - Opuscula Zoologica Fluminensia 165: 1-17.

Ross, H.H., 1956. Evolution and classification of the mountain caddisflies. - University of Illinois Press, Urbana, Illinois, USA. 213 pp.

Received: 26 June 2008

Accepted: 11 May 2009 